\title{
Fundamental Studies of Irradiation-Induced Defect Formation and Fission Product Dynamics in Oxide Fuels
}

\author{
DE-FC07-07ID14838 \\ University of Illinois at Urbana-Champaign \\ James Stubbins, PI \\ 1 June 2007 to 31 April 2012
}

\section{Final Technical Report}

19 December 2012

\author{
Principal Contributors \\ Weiying Chen, Yinbin Miao, Aaron Oaks, Bei Ye, Di Yun, Benjamin Holtzman, \\ Brian Kleinfeldt, Mark Kirk ${ }^{1}$, James Stubbins \\ Department of Nuclear, Plasma and Radiological Engineering \\ University of Illinois at Urbana-Champaign \\ ${ }^{1}$ Argonne National Laboratory
}




\section{EXECUTIVE SUMMARY}

The objective of this research program is to address major nuclear fuels performance issues for the design and use of oxide-type fuels in the current and advanced nuclear reactor applications. Fuel performance is a major issue for extending fuel burn-up which has the added advantage of reducing the used fuel waste stream. It will also be a significant issue with respect to developing advanced fuel cycle processes where it may be possible to incorporate minor actinides in various fuel forms so that they can be "burned" rather than join the used fuel waste stream. The potential to fission or transmute minor actinides and certain long-lived fission product isotopes would transform the high level waste storage strategy by removing the need to consider fuel storage on the millennium time scale.

For light water reactor (LWR) fuels, the potential of going to fuel burn-ups in excess of $100,000 \mathrm{MWd} / \mathrm{MT}$ is appealing, but limited by fuel restructure, burn-in of various fission products and cladding performance limitations. One are of high burn-up fuel performance that is not well understood is the "rimming" effect or restructuring of the fuel pellets in the outer rim volume of the fuel. A better understanding of the microstructural level of irradiation effects in oxides fuels is necessary to characterize irradiation-induced fuel restructuring processes and their effects on fuel performance.

The potential for the development of a minor actinide burning process was studied in past fast reactor programs and was under consideration during the past decade for a new fast burner reactor concept. The innovative part of mixed oxide (MOX) fuel used in the fast burner reactor concept is the incorporation of minor actinides in the $(\mathrm{U}, \mathrm{Pu}) \mathrm{O}_{2}$ phase, focusing directly on irradiation damage and fission product dynamics in these types of fuels since fuel performance will be the central issue for the performance of the system.

This study combined experimental and materials modeling techniques to study irradiation-induced microstructural effects in oxide fuels. The work consisted to a large extent of examining irradiation effects in $\mathrm{CeO}_{2}$ and $(\mathrm{Ce}, \mathrm{La}) \mathrm{O}_{2}$ as a function of temperature, dose, and composition. These material have comparable structures to $\mathrm{UO}_{2}$ and (U,Pu) $\mathrm{O}_{2}$ and are commonly used for surrogates for those materials. The addition of La has the added advantage of producing cation vacancy defects in a highly controllable way.

The experimental program relied heavily on ion irradiations with $\mathrm{Kr}$ and $\mathrm{Xe}$ of various energies where the damage level and the implantation level of the gas atoms could be controlled. The use of $\mathrm{Kr}$ and Xe mimics the effects of fission gases since these two elements are among the most frequently produced fission products and have a major impact on fuel restructuring and fuel physical properties. Many of the irradiation study results were obtained using the ANL IVEM-Tandem Microscope facility where the evolution of irradiation-induced microstructural changes could be observed in situ. This is a critically important experimental for studying the evolution of radiation-induced processes.

The results of the study show that it is possible to predict the development of the irradiation-induced microstructural changes which are largely due to the development and growth of the dislocation loop microstructure and the development of bubble structures. The dislocation loop characteristics were shown to be consistent with MD modeling results based on lowest energy loop configurations. 


\section{Table of Contents}

EXECUTIVE SUMMARY................................................................................................................. 0

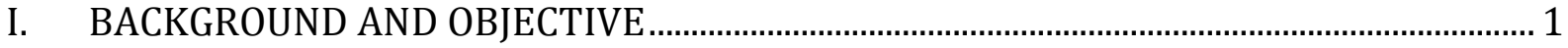

II. METHODOLOGY................................................................................................................... 2

A. Experimental Program............................................................................................................. 2

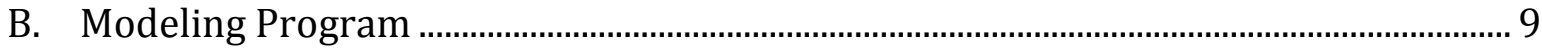

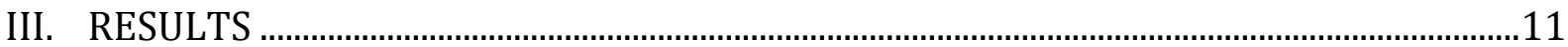

A. Temperature Effects ……………………………………………………………………....11

1. Evolution of dislocation structure ………………...................................................11

2. Gas Bubble Formation ................................................................................................19

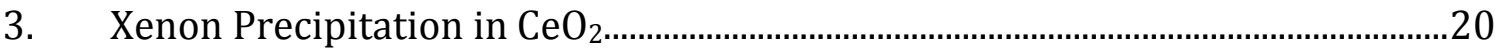

B. Stoichiometry Effects......................................................................................................23

1. Evolution of Dislocation Structures: Low Does Regime …………………………...24

2. Gas Bubble Formation: High Dose Regime.................................................................27

C. MD Simulation on Stoichiometry Effects on the Evolution of Dislocation Loops during Irradiation …………………………………………………………………………....34

D. MS, kMC Modeling of Stoichiometry Effects on Oxygen Diffusivity in $\mathrm{CeO}_{2} \ldots \ldots . . . . .39$

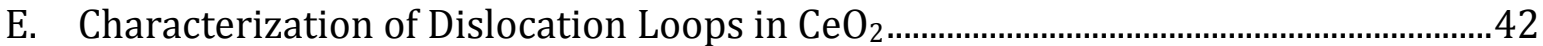

1. TEM Characterization...............................................................................................42

2. MD Simulation of the Atomistic Structure of the Dislocation Loops.....................46

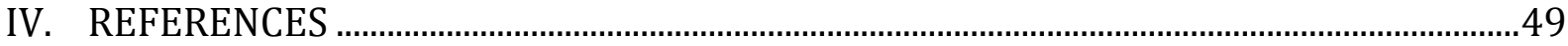

V. PUBLICATIONS AND PRESENATIONS ………………………………………………......52

VI. THESES AND DISSERTATIONS..............................................................................................55

VII. STUDENTS, DEGREES AND PLACEMENT …………............................................................56 
I. BACKGROUND AND OBJECTIVE

The objective of this research program is to address major nuclear fuels performance issues for the design and use of oxide-type fuels in the proposed Global Nuclear Energy Partnership (GNEP) a fast-spectrum Advanced Burner Test Reactor (ABTR) concept [1]. In the design and development of fast breeder reactors (FBR), one of the key challenges is the development of innovative fuel forms. Oxides, metals, nitrides and carbides are the major viable fuel forms envisaged for sodium-cooled fast reactors. Among these candidates, the oxide fuel is selected due to its satisfactory dimensional and radiation stability and chemical compatibility with cladding metals. In addition, it has the advantage of demonstrated and commercially available manufacturing technology with the least technical uncertainty. The innovative part of mixed oxide (MOX) fuel used in FBRs is the incorporation of minor actinides in the $(\mathrm{U}, \mathrm{Pu}) \mathrm{O}_{2}$ phase [1], focusing directly on irradiation damage and fission product dynamics in these types of fuels since fuel performance will be the central issue for the performance of the system.

Moreover, the pressure of competing economically with electricity produced from coal and natural gas has driven current reactor operator to seek even higher burnup of their fuel, generally $\mathrm{UO}_{2}$. The current fuel design has reached its limit at a burnup estimated to be $\sim 80$ $\mathrm{MWd} / \mathrm{kg} \mathrm{U}$. Extending the operational life of nuclear fuel in the reactor improves the efficiency of the materials flow process and results in a reduction of the cost of the fuel cycle. However, the discovery of high burnup structure (figure 1.1) in LWR spent fuels [2] raised some concern over the safety of extended fuel operation and highlighted the necessity of measuring and modeling the impact of the restructuring process on LWR fuel performance.
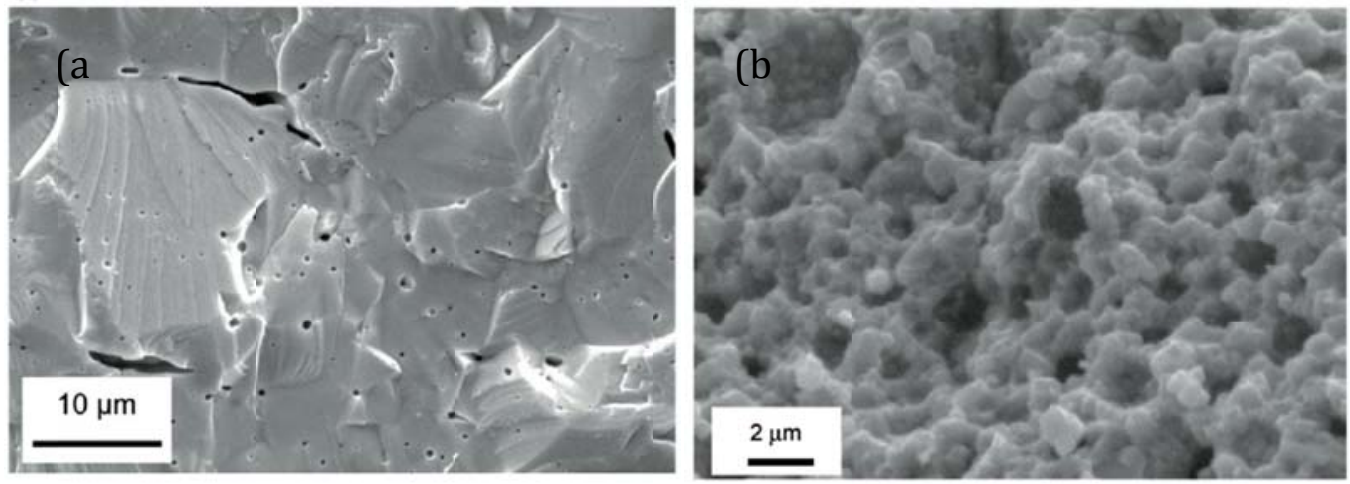

Figure 1.1 High magnification SEM fracture surface micrograph of $\mathrm{LWR} \mathrm{UO}_{2}$ fuel (a) before irradiation [2], (b) with a local burn-up of around $160 \mathrm{GWd} / \mathrm{tHM}$ [3].

The behavior of more complex oxides, such as MOX or TRU-MOX, has been given less attention, albeit due to the more complex issues in modeling. Nevertheless, this fuel form is of primary importance for ABTR success. In addition to the complex issues with (PuU) $\mathrm{O}_{2 \pm \mathrm{x}}$, similar issues with "impurity" element trapping, bonding and transport are of high interest. In fact, the TRU-type elements are typically highly substitutional for either $\mathrm{U}$ or $\mathrm{Pu}$, and several fission products, for example Mo, can compete with $\mathrm{U}$ or $\mathrm{Pu}$ as substitutional impurities. The objectives of this program are to examine and to model the irradiation effects in $\mathrm{CeO}_{2 \pm x}, \mathrm{UO}_{2 \pm x}$ and $(\mathrm{CeU}) \mathrm{O}_{2 \pm x}$ surrogate fuel types, the latter to initiate studies on mixed oxide systems for comparison with performance of MOX-type fuels. The irradiation effects were induced by ion implantation over a range on ion energies and to a range of ion doses to simulate the effects of fission product 
damage. The transport and trapping of simulated fission products were also examined. We have carried out using inert gas ions (e.g. $\mathrm{Kr}$ and $\mathrm{Xe}$ ) for both ion implantation to cause irradiation damage, and for dynamic transport studies to understand both trapping and defect mobility processes in these fuel forms.

Another objective of this program is to compliment experimental studies with modeling works using both molecular dynamics (MD) simulations of damage cascades in the oxide lattice as well as kinetic Monte Carlo (kMC) to study defect dynamics. The combination of experimental and modeling efforts have been extremely productive in understanding atomic displacement damage and effects in metal and metal alloy systems. The MD approach is extremely useful to understand the very early stages of irradiation damage defect structures during energetic displacement cascades under irradiation and $\mathrm{kMC}$ is very useful for using the defect configuration energies from MD to examine the defect and fission product transport mechanisms.

\section{METHODOLOGY}

The program is designed to develop an atomistic-level and microstructural-level understanding of irradiation effects and impurity atom dynamics in surrogate oxide nuclear fuels. This information will provide the basis for the development of microstructural-level fuel performance models which will be capable of providing the foundation for modeling TRU-MOX fuel behavior to high burn-ups and predictive models of fuel performance in off-normal events. The approach is divided into three sections:

1. Irradiation Performance of Simple Oxides - the program started by examining the irradiation behavior of simple fluorite oxides at stoichiometry and hypo- and hyper-stoichiometric ratios. The work was performed on $\mathrm{CeO}_{2 \pm x}$ and $\mathrm{UO}_{2 \pm x}$ in a well-characterized form. We used single crystal material to avoid grain boundary for ion irradiation/implantation experiments. We have examined both inert gas fission product types, $\mathrm{Kr}$ and $\mathrm{Xe}$ for various energies and temperatures.

2. Irradiation Performance of Complex Oxides - the work in this section employed more complex oxide forms, $(\mathrm{LaCe}) \mathrm{O}_{2-\mathrm{x}}$, to start to form an understanding of more complex oxides to simulate MOX-type structures and actinide behavior in this system. The experimental and modeling approach will be similar to that described for section 1 (above).

3. Fission Product Dynamics and Transport - the work in this section employed the as-irradiated specimens as a starting point for examining fission product trapping and transport in these oxide systems. The work relied on annealing and post-annealing examination of irradiated specimens to characterize "impurity" atom trapping and transport, as well as irradiationinduced defect configurations. The modeling work employed kinetic Monte Carlo (kMC) to characterize the atom transport mechanisms.

\section{A. Experimental Program}

In this study, krypton and xenon atoms were incorporated into the $\mathrm{CeO}_{2},(\mathrm{LaCe}) \mathrm{O}_{2}$ and $\mathrm{UO}_{2}$ crystals by ion implantation. This technique, combined with implantation computer simulation, provides good monitoring of the depth distribution and concentration of implanted species. It 
also allows for the fundamental diffusion processes to be studied individually by reduction unnecessary complications as much as possible.

Using ion irradiation instead of a test reactor environment to study irradiation damage is beneficial for studying basic damage processes, in addition to providing significant savings in time and money. Test reactor irradiation experiments can provide realistic irradiation environments for the study of material properties, but they are not amenable to studies involving a wide range of conditions, which is required for understanding the basic damage processes. In contrast, ion irradiation allows for variation of the irradiation parameters, such as dose, dose rate and temperature over a wide range of values. By carefully controlling those parameters, it is possible to precisely control the depth distribution and concentration of implanted species. A single cycle of reactor irradiation through microanalysis can take 3 5 years. Such a long cycle length reduces flexibility in altering irradiation programs. In addition, the long cycle length together with the special requirements of facilities and sample handling can result in very high costs for reactor irradiation experiments. Ion irradiation normally only requires on the order of tens of hours to reach desired damage levels, and produces little or no residual radioactivity, allowing handling of samples without the need for special precautions. All of these features make it a very attractive technique in studying radiation effects in nuclear materials.

There were two ion implantation facilities used in this study. The high voltage engineering Van de Graaff is an accelerator located in Materials Research Laboratory at the University of Illinois (figure 1.2). This accelerator was employed to irradiate samples that were to be analyzed using ex situ irradiation. The Van de Graaff operates at energies up to $2.3 \mathrm{MeV}$ for $\mathrm{H}, \mathrm{He}, \mathrm{Ar}, \mathrm{Kr}$, $\mathrm{Xe}$ and Ne with 3-4-5 mm beam-size apertures in shapes of circular or square. The sample stages allow the irradiation temperature to be controlled in the range of room temperature to $800^{\circ} \mathrm{C}$.

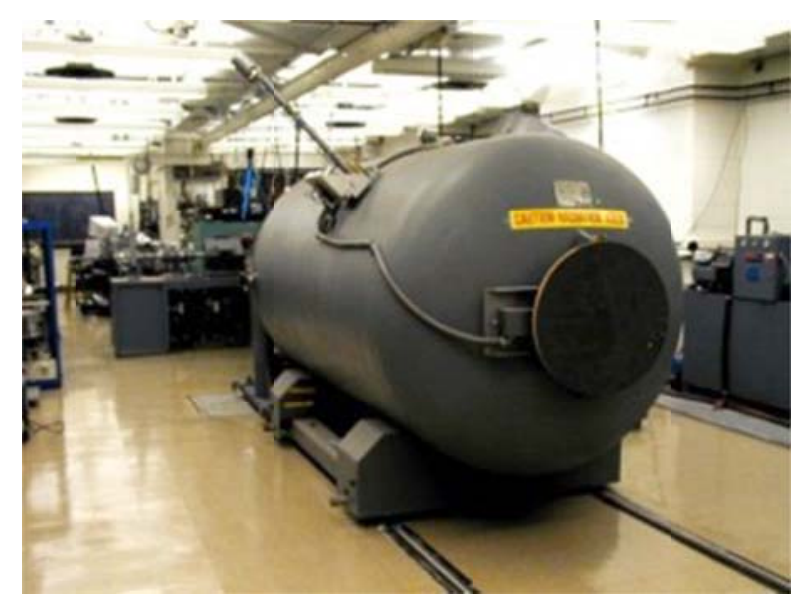

Figure 1.2 High voltage engineering Van de Graaff accelerator at the University of Illinois 
The other irradiation facility used in this study was IVEM-Tandem facility at Argonne National Laboratory, as shown in figure V.5. All of the in situ irradiation experiments were carried out on this instrument. It is a transmission electron microscope interfaced with an ion accelerator for in situ ion beam studies involving ion implementation and/or ion damage.
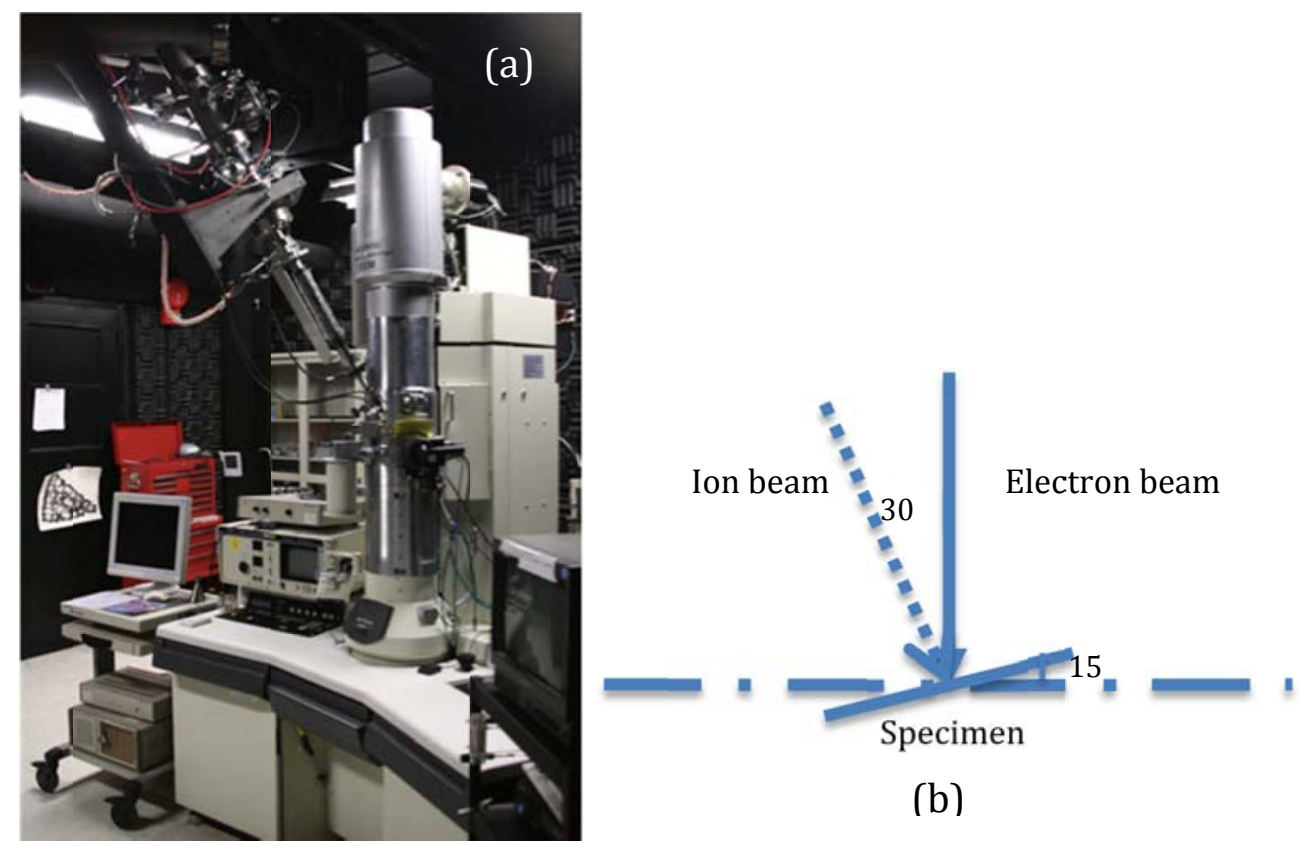

(b)

Figure 1.3 (a) IVEM-TANDEM facility at Argonne National Laboratory, USA [4]; (b) Schematic diagram of ion beam and electron beam in IVEM-Tandem. The specimen is normally tilted $15^{\circ}$ in the $y$ direction during irradiation.

The IVEM is a $300 \mathrm{kV}$ Hitachi H-9000NAR with the ion beam entering at $30^{\circ}$ to the electron beam. The objective pole gap was widened to allow the incorporation of the ion beam but with a much smaller detrimental effect on resolution. The ion flux is monitored constantly during irradiation. In addition to a variety of specimen holders allows in situ cooling and heating experiments (with or without ion irradiation) from 15-1200K. Data recording is provided by choices of film, a Gatan 622 video rate camera, and a Gatan Orius SC 1000 CCD camera with Digital Micrograph software.

The available ion sources include noble gases, $\mathrm{H}, \mathrm{S}, \mathrm{Fe}, \mathrm{Ni}, \mathrm{Cu}$ and $\mathrm{Au}$, and the typical flux at the specimen is $1 \times 10^{12} 1 / \mathrm{cm}^{2} \mathrm{~s}$. The ion-beam is raster scanned across an aperture in the ion beam line just before it enters the TEM column which is giving rise to a uniform irradiated area on the sample of a $2 \mathrm{~mm}$ diameter circle in the center of a standard $3 \mathrm{~mm}$ TEM specimen. The $30^{\circ}$ incline angle permits continuous observation and data recording during irradiation. As shown in figure 1.3 (b), the specimen is tilted $\sim 15^{\circ}$ facing the ion beam to assure enough exposure area on the specimen to the ion beam.

The goal of this study is to understand the formation processes of defect structures. Therefore the irradiation experiments were designed to achieve the desired damage level and gas atom concentration. There are several variable parameters in the selection of irradiation conditions: ion source and energy, irradiation temperature, and dose. Irradiation damage levels 
were estimated with SRIM 2008 [5]. In these calculations, $\mathrm{E}_{\mathrm{d}}=51 \mathrm{eV}$ was used for $\mathrm{Ce}$ displacement energy instead of $E_{d}=25 \mathrm{eV}$ which is the default value in SRIM. This value is measured by Yasunaga et al. [6]. In their experiments of electron irradiation, Ce displacement energies of 44 to $58 \mathrm{eV}$ were measured. Ion distributions calculated with SRIM are displayed in figure 1.4 .
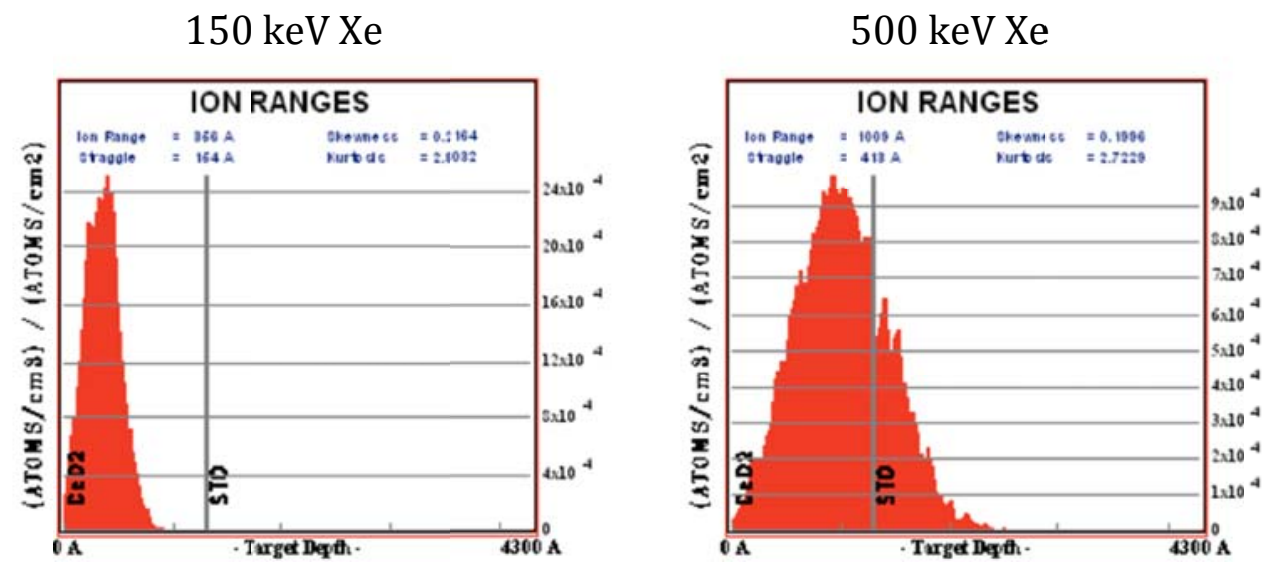

$150 \mathrm{keV} \mathrm{Kr}$

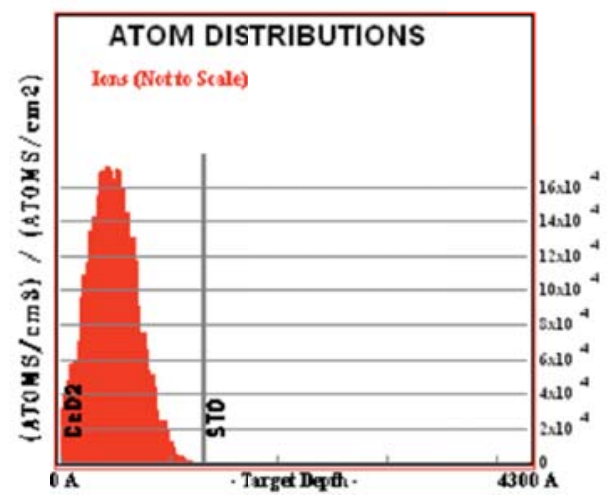

$1 \mathrm{MeV} \mathrm{Kr}$

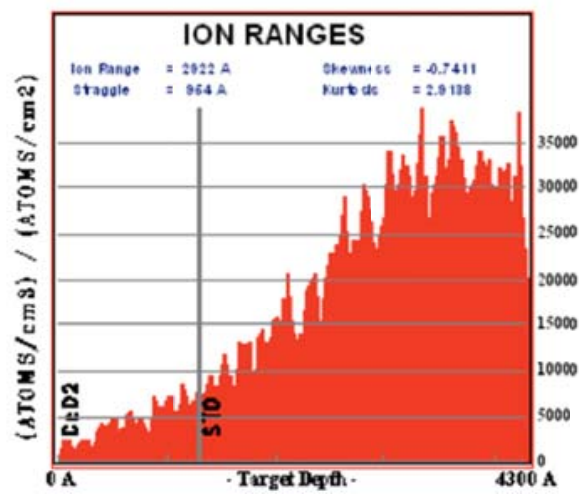

Figure 1.4 Ion distributions in $\mathrm{CeO}_{2}$ thin films for different ion species and energies.

All of the TEM specimens in this study were prepared in MRL at the University of Illinois. TEM sample preparation is a rather complex procedure. TEM specimens are required to be at most hundreds of nanometers thick to allow the electron beam to transmit. The thickness must be comparable to the mean free path of the electrons that travel through the samples, which may be only a few ten of nanometers. Extra care is required during the entire process of making and handling TEM specimens due to safety concerns and their fragility. Preparation of TEM specimens is specific to the material to be analyzed and the desired information to be obtained from the specimen. The specimen preparation processes in this study includes three main steps:

i. Cut the bulk sample into proper size, normally in stripes with a dimension of $2 \mathrm{~mm} \times 4 \mathrm{~mm}$.

ii. Mechanical thinning to make a slice of material between 30-50 $\mu \mathrm{m}$ thick with boron carbide powder and diamond lapping film.

iii. Final thinning and polishing to achieve desired thickness $(<100 \mathrm{~nm})$ in thin area by ion milling. 
The bulk material initially provided is in a square shape with a size of $14 \mathrm{~mm} \times 14 \mathrm{~mm}$. The specimen material is ceramic, so it is reasonably hard and mechanical damage is not crucial. A diamond saw (figure 1.5) is used to cut the stripes from the as-grown material. In order to section the sample precisely, the sample is adhered to a stage with crystalbond, and the size of sectioned piece can be read from an advanced digital micrometer. After slicing, the sectioned stripes need to be cleaned with acetone in an ultrasonic cleaner to remove residue crystalbond and lubricant oil.

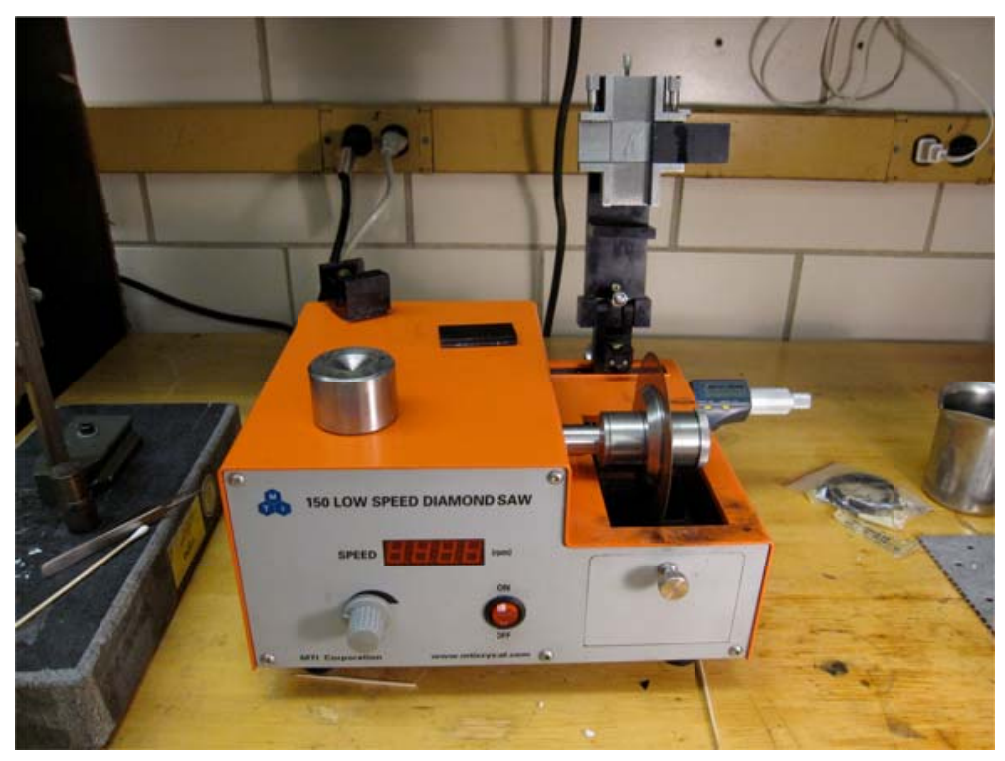

Figure 1.5 Low speed diamond saw located in Materials Research Laboratory at the University of Illinois

Two types of TEM specimens are prepared: cross-section specimens and plan view specimens. Cross-section specimens are prepared from the ex situ irradiated materials to better review ion distribution depth profile, and plan view specimens are prepared for in situ irradiation for providing better stability.

Cross-section specimen is a special type of specimen. Rather than trying to thin one interface only, the sample need to be cut (figure 1.6(a)), and glued together with M-bond with two films facing each other to form sandwich like structure (figure 1.6(B)). After curing with a hot plate at $120^{\circ} \mathrm{C}$ until M-bon solidifies, it can be mounted on a tripod for mechanical thinning (figure 1.6(c)). In this process, the gluing of the sections to form the sandwich is a critical step. It can protect the thin film areas in the following thinning processes. 


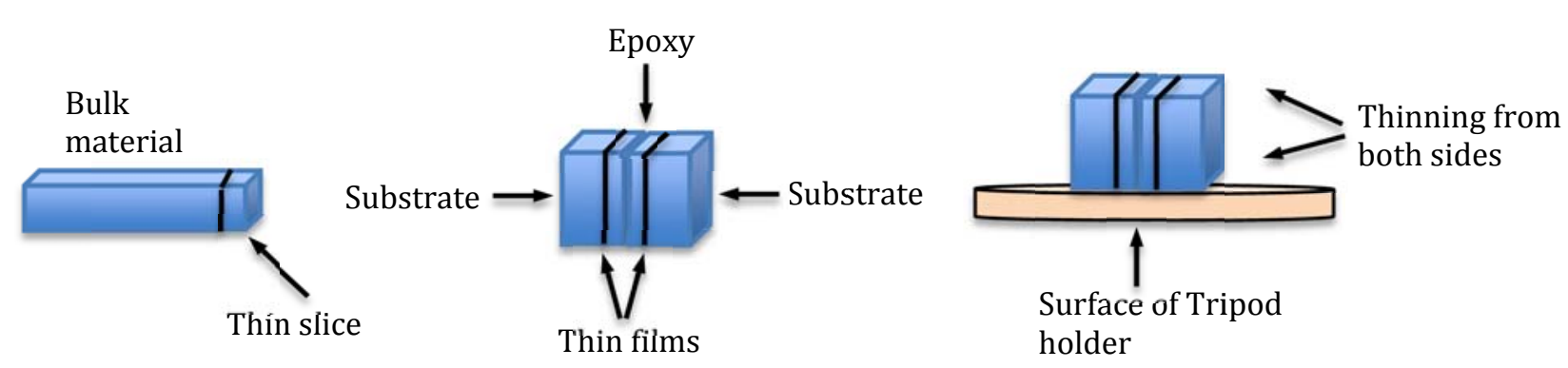

(a)

(b)

(c)

Figure 1.6 Sequence of steps for preparing cross sectional TEM specimens.

Mechanical thinning needs to be done to a high quality to ensure constant sample thickness across the region of interest. This is achieved by tripod thinning on boron carbide power to a thickness of 50-60 $\mu \mathrm{m}$. Subsequently, it has to be followed with further thinning and final polishing on diamond lapping films. The final thickness is $\sim 30-40 \mu \mathrm{m}$, and the fine polishing can remove scratches which are produced during grinding and may cause contrast fluctuations in TEM observation. Tripod polishing can be used to prepare sections in all orientations (planar longitudinal and cross section orientations). This technique is best suited to hard, brittle and multiphased materials. It consists in two main tools: a specimen holder (figure 1.7) and a polishing machine (figure 1.8). The specimen holder is adjusted by means of three micrometer screws ("tripod"). The diamond lapping films are placed on the low speed rotary polisher, and polishing is carried out using water as a lubricant. In this step, the stacked sandwich-like slices for cross sectional samples are thinned and polished from both sides, and the pieces for plan view samples are thinned only from the substrate side.
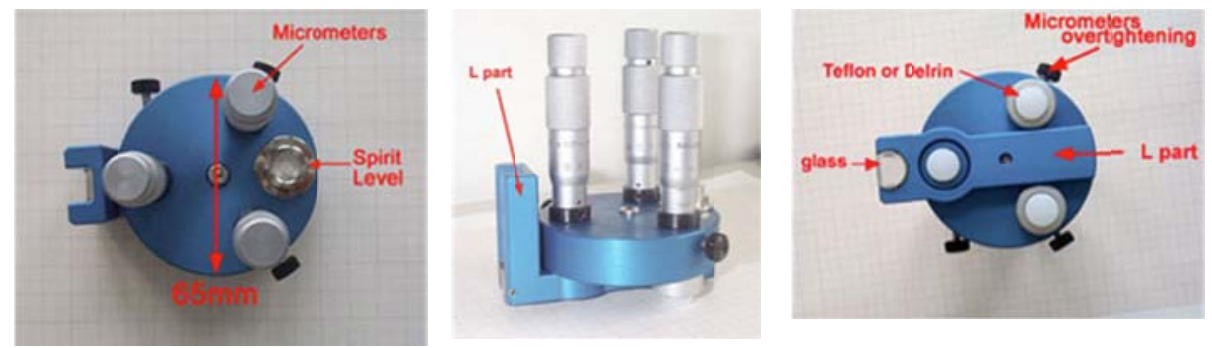

Figure 1.7 The top, side and bottom view of a tripod specimen holder 


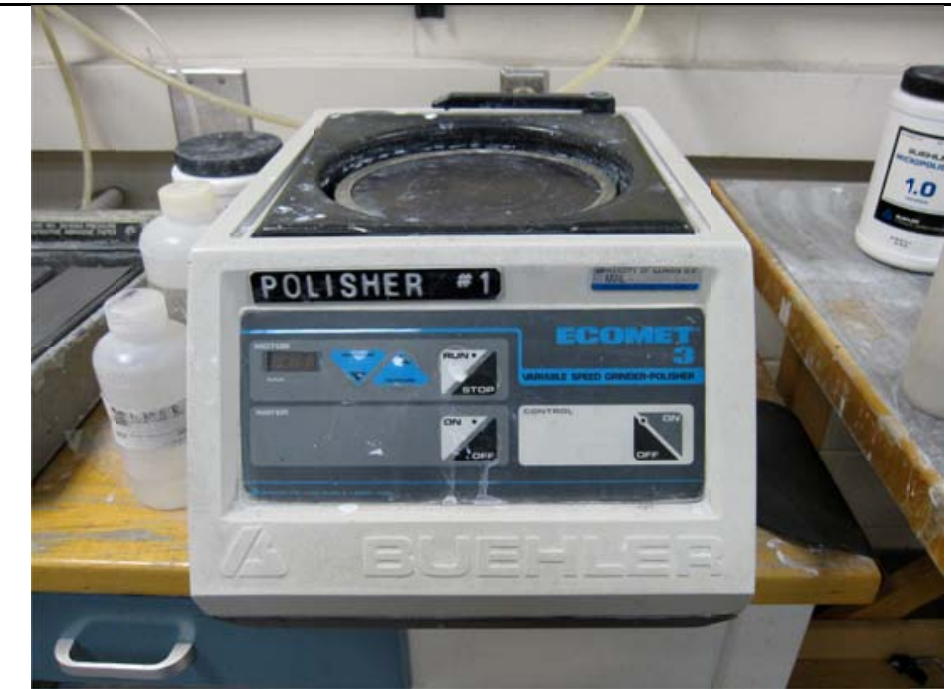

Figure 1.8 The polisher located in the Materials Research Laboratory at the University of Illinois

For the ion milling process, a GATAN 691 Precision Ion Polishing System (PIPS) (figure 1.9) is used. In this machine, two focused Ar ion beams mill the pre-thinned sample in such a way that a hole results at the desired position. In general, the parameters for the ion milling process are specific for each material and have to be optimized. The ion milling rate increases with higher etching angle and higher etching voltage; however, the sample is also more severely damaged. Therefore, the angle, as well as the voltage, should be kept rather low to minimize the damage induce by ion beams. Generally, a higher voltage combined with lower angle is less harmful to the sample than lower voltage combined with higher angle. In this thinning process, an accelerating voltage of $5 \mathrm{keV}$ and an incline angle of $6-7^{\circ}$ were chosen to achieve perforation. The accelerating voltage was then reduced to $3 \mathrm{keV}$ for final polishing to remove deposited material on the sample surface. The sample is rotated during etching to promote even polishing of the sample surface.

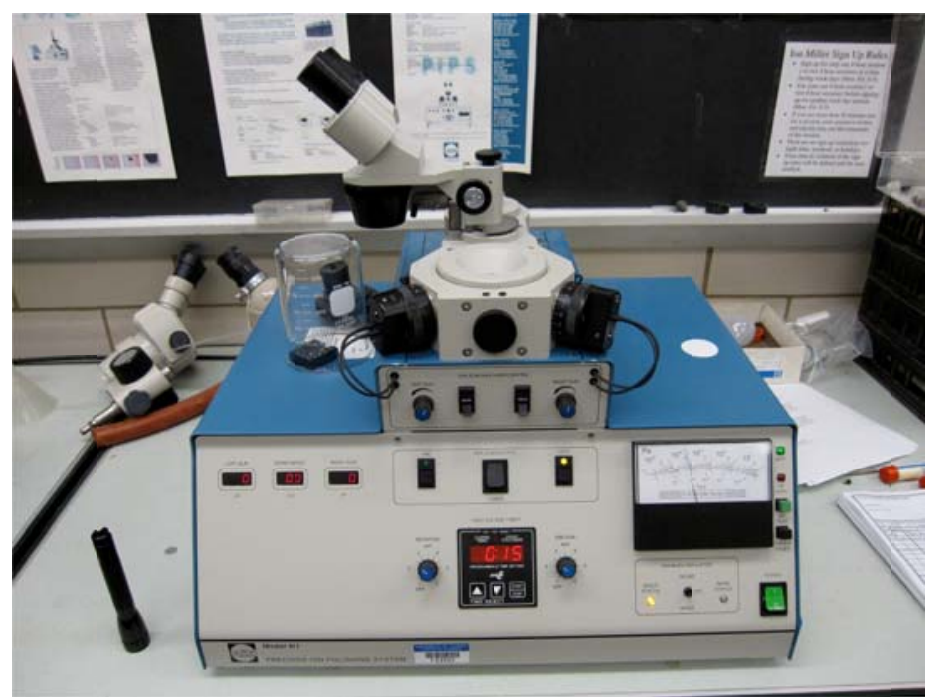

Figure 1.9 GATAN 681 PIPS ion miller located in the Materials Research Laboratory at the University of Illinois 
For a cross sectional TEM sample, its position on PIPS specimen holder is shown in figure 1.10. During ion milling, one of the ion beams etches from the top (incline angle $=+7^{\circ}$ ), while the other etches from the bottom (incline angle $=-7^{\circ}$ ). This is done to eliminate the re-deposition layers on both sides of the specimen. Two ion beams are aligned to focus on the center of the specimen. In this case, the modulator is switched to "double" mode, which ensures each ion beam works only half of the cycle in each stage rotation. For a plan view TEM sample, the specimen is mounted on a sample stage (figure 1.10(b)) with Crystalbond with the substrate side facing up. Two ion beams etch from the top at the same time and are focused on the center. The modulator is turned off, so the sample will be etched continuously. It is necessary to coat the film (down) side with a thin layer of protector (here is Crystalbond) for a plan view specimen, and then dissolve this coating after thinning to remove sputtered material. The ion milling time varies with the thickness of the pre-thinned sample. Typically, it takes $\sim 3$ hours to perforation and another 15 minutes for fine polishing.

(a)

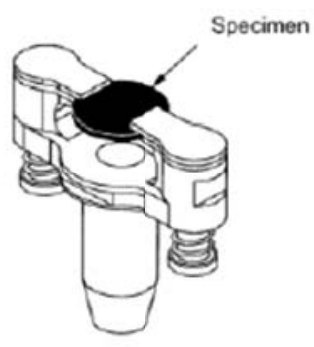

(b)

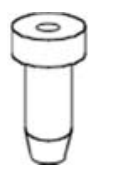

Figure 1.10 (a) TEM specimens on a clamp-type sample holder for ion milling of cross-section TEM specimens, (b) a post-type sample holder for ion milling of plan view TEM specimens

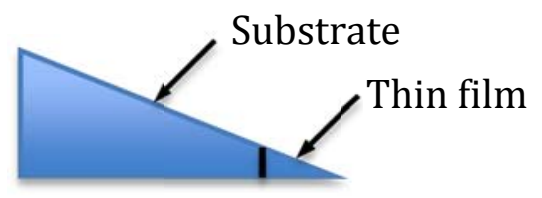

(a)

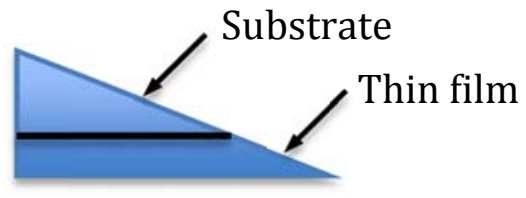

(b)

Figure 1.11 Schematic drawings of thin areas in (a) cross-section and (b) plan-view TEM specimens after ion milling.

\section{B. Modeling Program}

For the modeling part of this study, three major techniques, which are molecular statics (MS), molecular dynamics (MD) and kinetic Monte Carlo (kMC) are utilized.

As classical atomistic simulations, the accuracy and reliability of both molecular statics and dynamics simulations mainly depend on the selection of the potential, which describes the atomistic interactions. In this study, the Buckingham potential form with Coulomb term is applied for our ceria system with lanthanum dopant. The Buckingham potential is a pair potential, which means the interaction energy between each pair of the atoms in the system only relies on the separation distance. The potential has the following form, 


$$
\mathrm{U}(\mathrm{r})=\mathrm{A} e^{-r / \rho}-\frac{C}{r^{6}}+\frac{q_{1} q_{2}}{4 \pi \varepsilon_{0} r}
$$

where, $\mathrm{A}, \rho$ and $\mathrm{C}$ are the three potential parameters, $r$ is the separation distance and the last term accounts for the Coulomb potential.

Once the potential is determined, the total potential energy of a certain system is the summation of the potential energy of every atom pair inside it. Hence, the total potential energy is a function of the coordinates of atoms. Thus, the minimum point and first-order saddle point of this function respectively correspond to the optimal and migration transitional state of the system. By means of Newton-Ralphson optimizer and rational functional optimizer (RFO), these stationary points can be obtained numerically so that the optimal defect energy and the defect migration energy can be calculated. This is the fundamental principle of the molecular statics calculation. In this study, GULP code is used to achieve all the statics calculation work.

For the MD simulations, the kinetic energy of the system is included into the total system energy, namely, the velocities of atoms are considered, which change with time according to the Newton's second law. Therefore, the atomistic positions evolve with time as follows by using the Taylor's series.

$$
\vec{x}^{p}(t+d t)=\vec{x}(t)+\vec{v}(t) d t+\frac{1}{2 !} \vec{a}(t) d t^{2}+\frac{1}{3 !} \frac{d^{3} \vec{x}(t)}{d t^{3}} d t^{3}+\frac{1}{4 !} \frac{d^{4} \vec{x}(t)}{d t^{4}} d t^{4}+\frac{1}{5 !} \frac{d^{5} \vec{x}(t)}{d t^{5}} d t^{5}
$$

This is called the integrator of MD simulation. Complex correction techniques are used to make the integrator more convergent so that larger time step can be chosen to save the computation time. Also, with the kinetic energy, the temperature of the system can also be defined,

$$
\left[E_{k}\right]=\frac{3}{2} k_{B} T
$$

More importantly, the system temperature can be controlled by modifying the atomistic velocities. The pressure of the system can also be controlled using similar algorithms. The mainstream thermostat and barostat are based on the rescaling or Nose-Hoover method.

Different from atomistic simulation, the fundamental element of kMC simulation is the incident rather than the atom. In our case, the incident is the defect migration. For a certain incident $i$, the rate is defined by Arrhenius equation,

$$
\Gamma_{i}=v_{o} \exp \left(\frac{E_{m i g}^{i}}{k_{b} T}\right)
$$

where, $\Gamma_{i}$ is the rate, $v_{o}$ is the Debye frequency, $E_{m i g}^{i}$ is the energy barrier of incident $\mathrm{i}, k_{b}$ is Boltzmann constant and $T$ is the temperature. Then the cumulative rate is defined as follows,

$$
R_{i}=\sum_{j=1}^{i} \Gamma_{j}
$$

The total incident number is $\mathrm{N}$. Then a random number $\rho_{1} \in(0,1]$ is generated to determine which incident takes place. For example, incident i happens when $R_{i-1}<\rho_{1} R_{N} \leq R_{i}$. Thus, another random number $\rho_{2} \in(0,1]$ is generated to decide the advanced time clock,

$$
\Delta \mathrm{t}=\frac{-\ln \left(\rho_{2}\right)}{R_{N}}
$$


Then, the system can evolve with time.

For all the three simulation methods, periodical boundary condition is essential for modeling the bulk properties instead of the surface effects. Also, the detailed setups of the systems are described in the corresponding result part of each specific project.

\section{RESULTS}

The results are composed of four parts: (a) the temperature effects: studying the behavior of defect structure (dislocations, voids/bubbles) evolution in $\mathrm{CeO} 2$ and $\mathrm{UO} 2$ under $\mathrm{Xe}$ and $\mathrm{Kr}$ irradiations at various temperatures. (b) The stoichiometry effects: studying the behavior of defect structure evolution in $\mathrm{CeO} 2$ doped with various amount of La, under similar irradiation and temperature conditions as in part (a). (c) Characterization of dislocation loops: determining several properties of dislocation loops formed by irradiations in $\mathrm{CeO} 2$ such as loop plane, burgers' vector and the nature of the loops. (d) Measurement of radiation enhanced diffusivity: a cooperation with Prof. Heuser group in university of Illinois studying the diffusivity of La in $\mathrm{CeO} 2$ under irradiation.

\section{A. Temperature Effects}

\section{Evolution of dislocation structure}

Figure 2.1 shows the sequential change of defect cluster damage in $\mathrm{CeO}_{2}$ with $500 \mathrm{keV} \mathrm{Xe}$ ions at $800^{\circ} \mathrm{C}$. This dose sequence was taken during irradiation in the ANL IVEM/Tandem facility where the dose levels could be tracked in the same sample area. The choice of $800^{\circ} \mathrm{C}$ irradiation temperature is sufficiently high that 0 defects should be mobile as should vacancies on the 0 sublattice.

The evolution of a fine defect structure was evident in the microstructure. In figure 2.1, image (a) and (b) show the microstructure of the specimen before irradiation as prepared and annealed respectively. There were defect clusters to begin with (black contrasts), which were induced by the ion milling process. The defect clusters changed from irregular shapes to almost circular ones after annealing. This shows defects become mobile when temperature rises. The exact characteristic of these defect clusters is not yet clear.

To understand the nature of the defect clusters, $g \bullet b$ analysis and trace analysis will be applied in the future. From previous studies [7], it is believed that interstitials generated during irradiation displacement are inclined to form dislocation loops. Furthermore, the relatively excessive vacancies left in the matrix group into voids, which provide perfect traps for gas atoms.

The size of defect structures, which were characterized as dislocation loops, grew with increasing dose $\left(0-3 \times 10^{14}\right.$ ions $\left./ \mathrm{cm}^{2}\right)$, as displayed in figure 2.1 (c-e). Meanwhile, the density of defect clusters remained nearly unchanged. This suggests that nucleation of defect clusters is completed in a very early stage of irradiation (during ion milling in this case). A similar result was observed in $\mathrm{CeO}_{2}$ irradiated with electrons [8]. 
Fundamental Studies of Irradiation-Induced Defect Formation and Fission Product Dynamics in Oxide Fuels
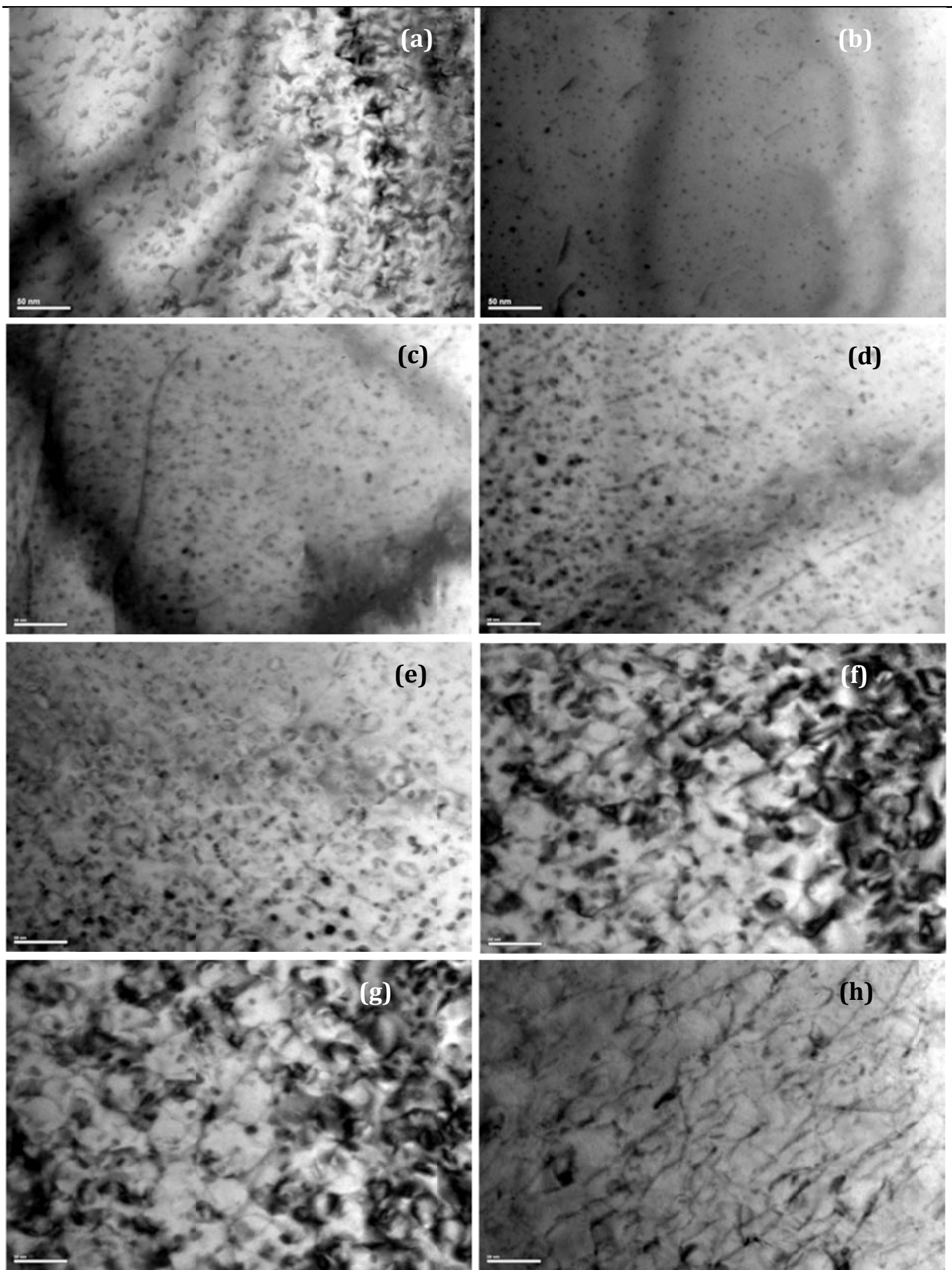

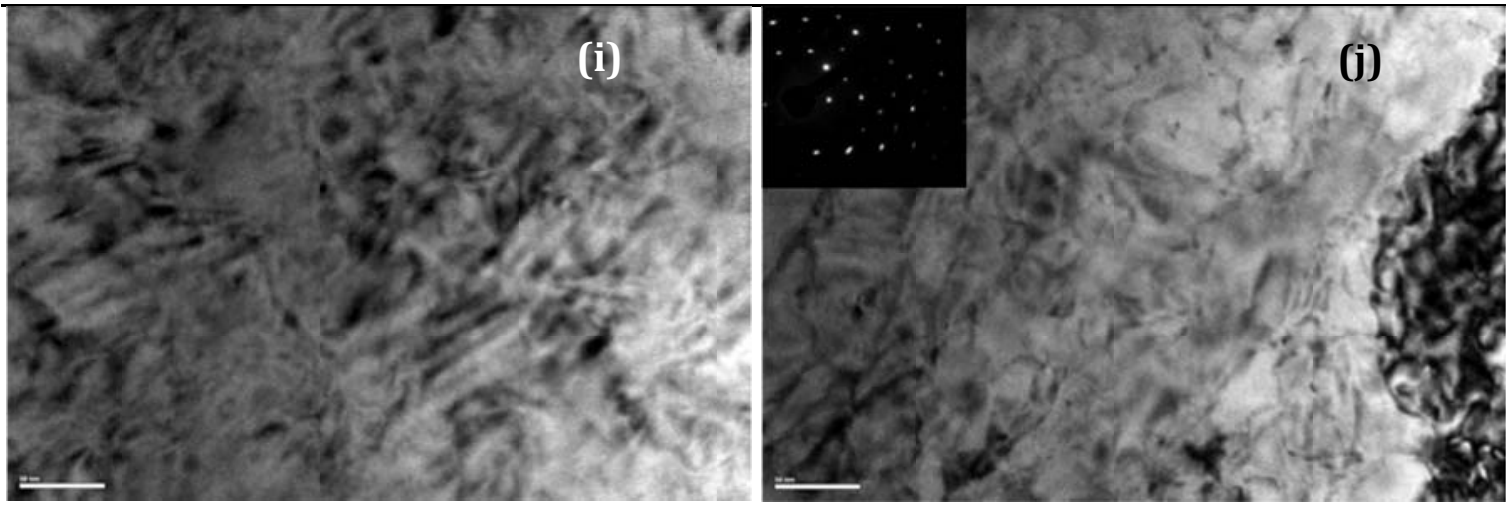

Figure 2.1 Bright-field TEM micrographs recorded during the in situ irradiation of single crystal $\mathrm{CeO}_{2}$ with $500 \mathrm{keV} \mathrm{Xe}$ ions at $800^{\circ} \mathrm{C}$. The applied flux is $1.25 \times 10^{9}$ ions $/ \mathrm{cm}^{2} \mathrm{~s}$ before $3 \times 10^{14}$ ions $/ \mathrm{cm}^{2}$ and $6.25 \times 10^{9}$ ions $/ \mathrm{cm}^{2} \mathrm{~s}$ after that. The corresponding dose are (a) 0 (as prepared) (b) 0 (annealed) (c) $5 \times 10^{13}$ (d) $1 \times 10^{14}$ (e) $3 \times 10^{14}$ (f) $8 \times 10^{14}$ (g) $1 \times 10^{15}$ (h) $1.8 \times 10^{15}$ (i) $3 \times 10^{15}$ (j) $5 \times 10^{15}$ in unit of ions $/ \mathrm{cm}^{2}$. The scale length is $50 \mathrm{~nm}$.

When the dose reached $8 \times 10^{14}$ ions $/ \mathrm{cm}^{2}$ (figure 2,1 (f)), dislocation loops became much less distinguishable. Some of them merged with each other and developed into micro-cells walled with extended dislocation loops. This process continued through a dose of $1 \times 10^{15}$ ions $/ \mathrm{cm}^{2}$ (figure $2.1(\mathrm{~g})$ ). Finally tangled dislocation networks clearly appeared when the dose reached $1.8 \times 10^{15}$ ions $/ \mathrm{cm}^{2}$ (figure 2.1). The same processes occurred during in situ irradiation of $390 \mathrm{keV}$ $\mathrm{Xe}$ and $300 \mathrm{keV} \mathrm{Cs}$ in $\mathrm{UO}_{2}$ [9].

When incremental implantation went beyond $1.8 \times 10^{15}$ ions $/ \mathrm{cm}^{2}$, it became difficult to maintain the same diffraction condition during TEM observation due to sample bending. The detailed defect structure, shown in figure 2.1 (i, j), was difficult to clearly resolve. Nevertheless by comparing figure 2.1 (i) and (j), it can be seen that the defect structure approached a stable configuration by this stage of irradiation. The inset in figure 2.1 (j) is a diffraction pattern taken after the irradiated specimen was cooled to room temperature. The round diffraction spots demonstrated the specimen remains crystalline.

The evolution and growth behavior of irradiation-induced defect structures was studied as a function of dose during incremental irradiation of $\mathrm{CeO}_{2}$ with $500 \mathrm{keV} \mathrm{Xe}$ at $800^{\circ} \mathrm{C}$ using in situ TEM techniques.

The evolution process of the dislocation structure developed through several stages:

1. Nucleation of dislocation loops was completed at the very early stage of irradiation. For this case, it was finished during ion milling process.

2. With the increasing dose, dislocation loops expanded radically and began to interact with each other. Some of them coalesced, which accelerated loop growth.

3. By the time the dose reached $1.8 \times 10^{15}$ ions $/ \mathrm{cm}^{2}$, a tangled dislocation network had evolved in the specimen.

4. Following the evolution of the dislocation network structure, the microstructure remained stable to higher doses. 
Two series of bright-field TEM micrographs taken on single crystal $\mathrm{CeO}_{2}$ during in-situ irradiations of $1 \mathrm{MeV} \mathrm{Kr}$ at $600^{\circ} \mathrm{C}$ and $800^{\circ} \mathrm{C}$ are presented in figure 2.2 (a1) - (a7) and (b1) (b7) respectively. Figure 2.2 shows the sequential change of microstructure of the same areas as a function of dose: from the condition before irradiation to an accumulated dose of $5 \times 10^{15}$ ions $/ \mathrm{cm}^{2}$. The microstructures acquired at different irradiation temperatures with the same ion irradiation dose are displayed side by side.
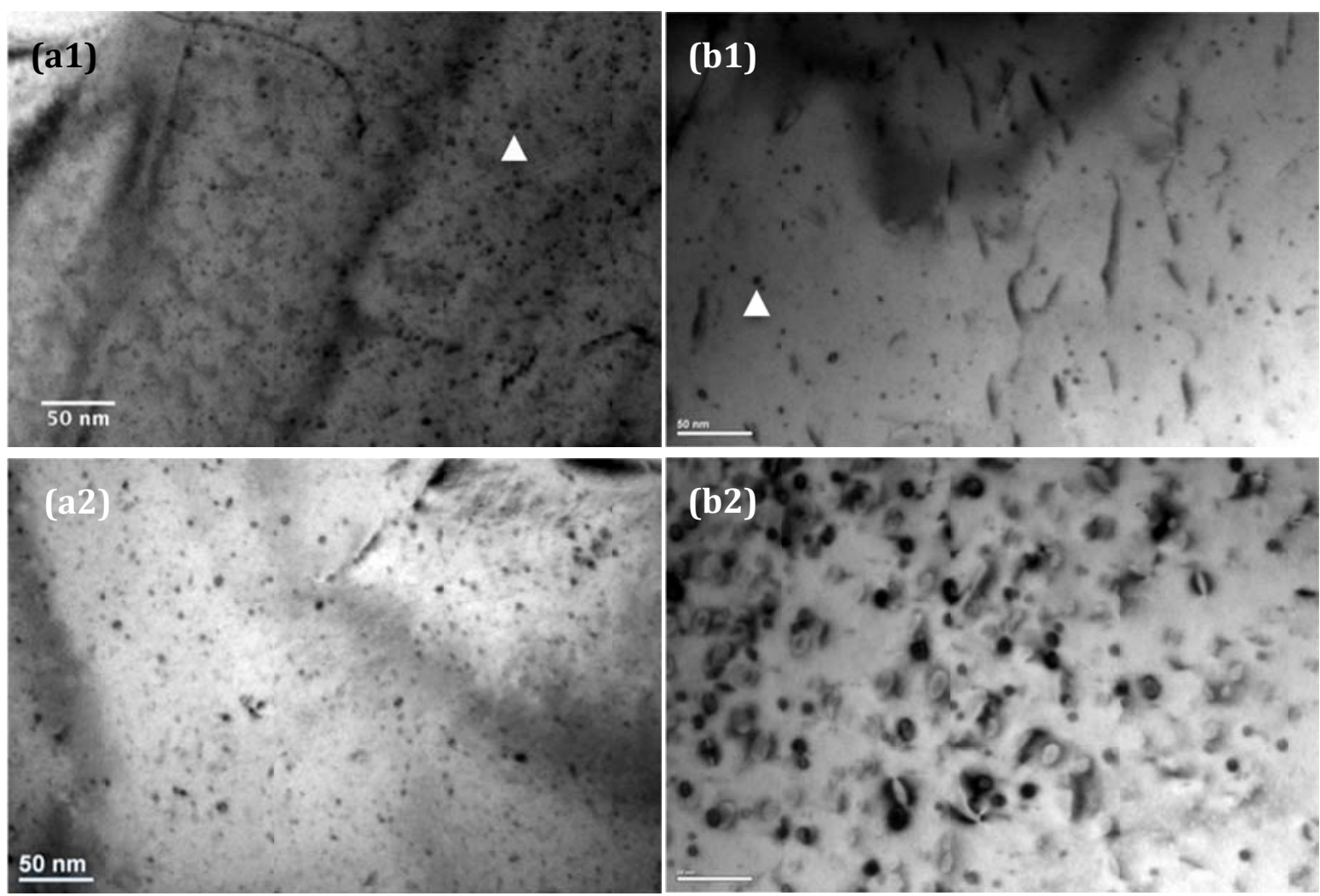

Figure 2.2. Sequential change in the nucleation and growth of defect clusters in singe crystal $\mathrm{CeO}_{2}$ irradiated with $1 \mathrm{MeV} \mathrm{Kr}$ at $600^{\circ} \mathrm{C}$ (left column) and $800^{\circ} \mathrm{C}$ (right column) at various dose levels: (a1) and (b1) before irradiation, (a2) and (b2) $3 \times 10^{14}$ ions $/ \mathrm{cm}^{2}$. The observations were carried out from the [001] direction with $g=220$ reflection. 
Fundamental Studies of Irradiation-Induced Defect Formation and Fission Product Dynamics in Oxide Fuels
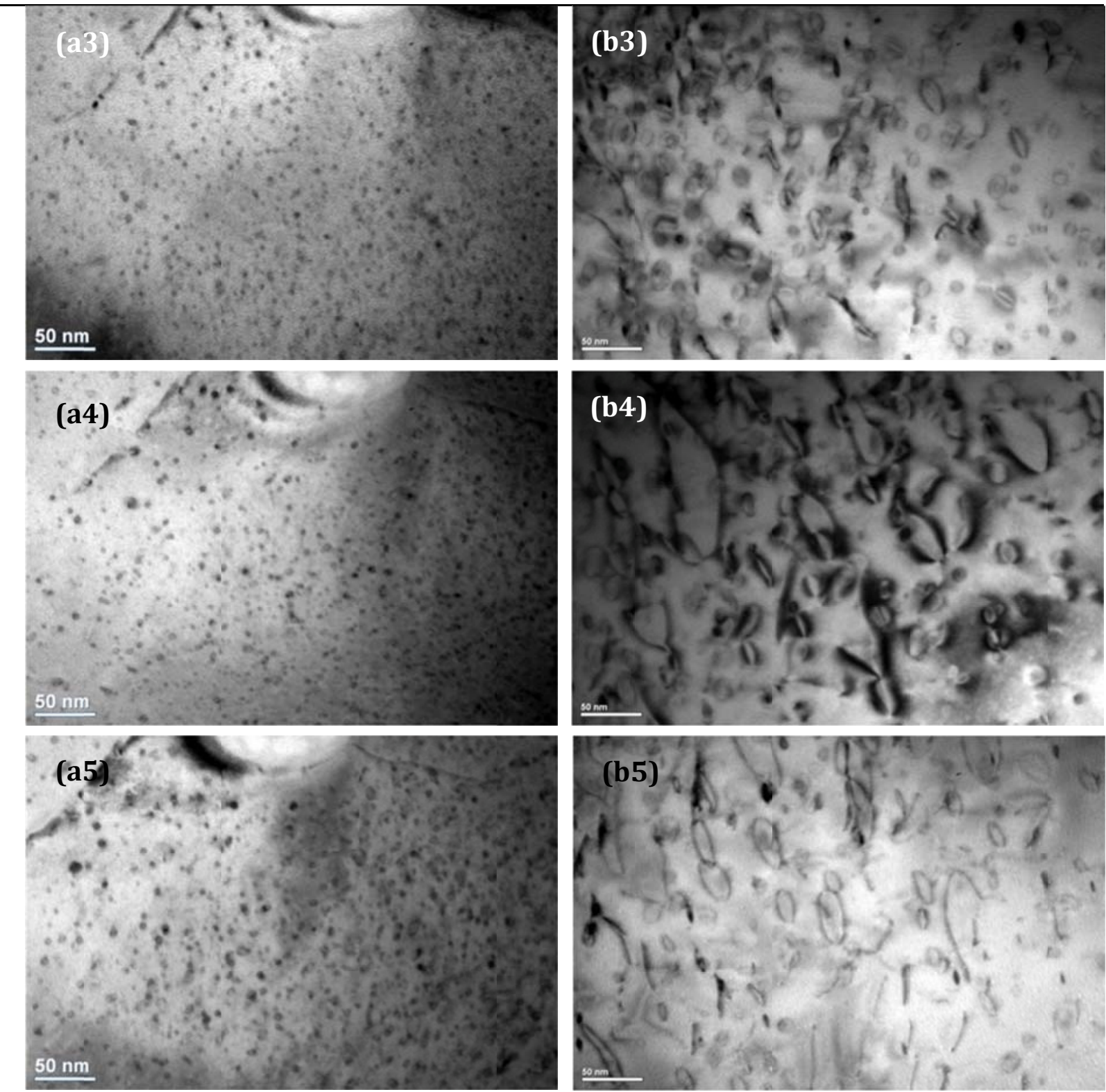

Figure 2.2 (cont.) Sequential change in the nucleation and growth of defect clusters in singe crystal $\mathrm{CeO}_{2}$ irradiated with $1 \mathrm{MeV} \mathrm{Kr}$ at $600^{\circ} \mathrm{C}$ (left column) and $800^{\circ} \mathrm{C}$ (right column) at various

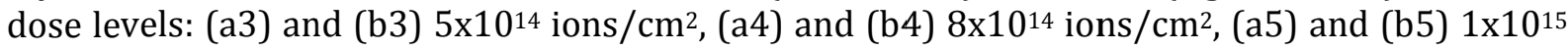
ions $/ \mathrm{cm}^{2}$. The observations were carried out from the [001] direction with $g=220$ reflection. 

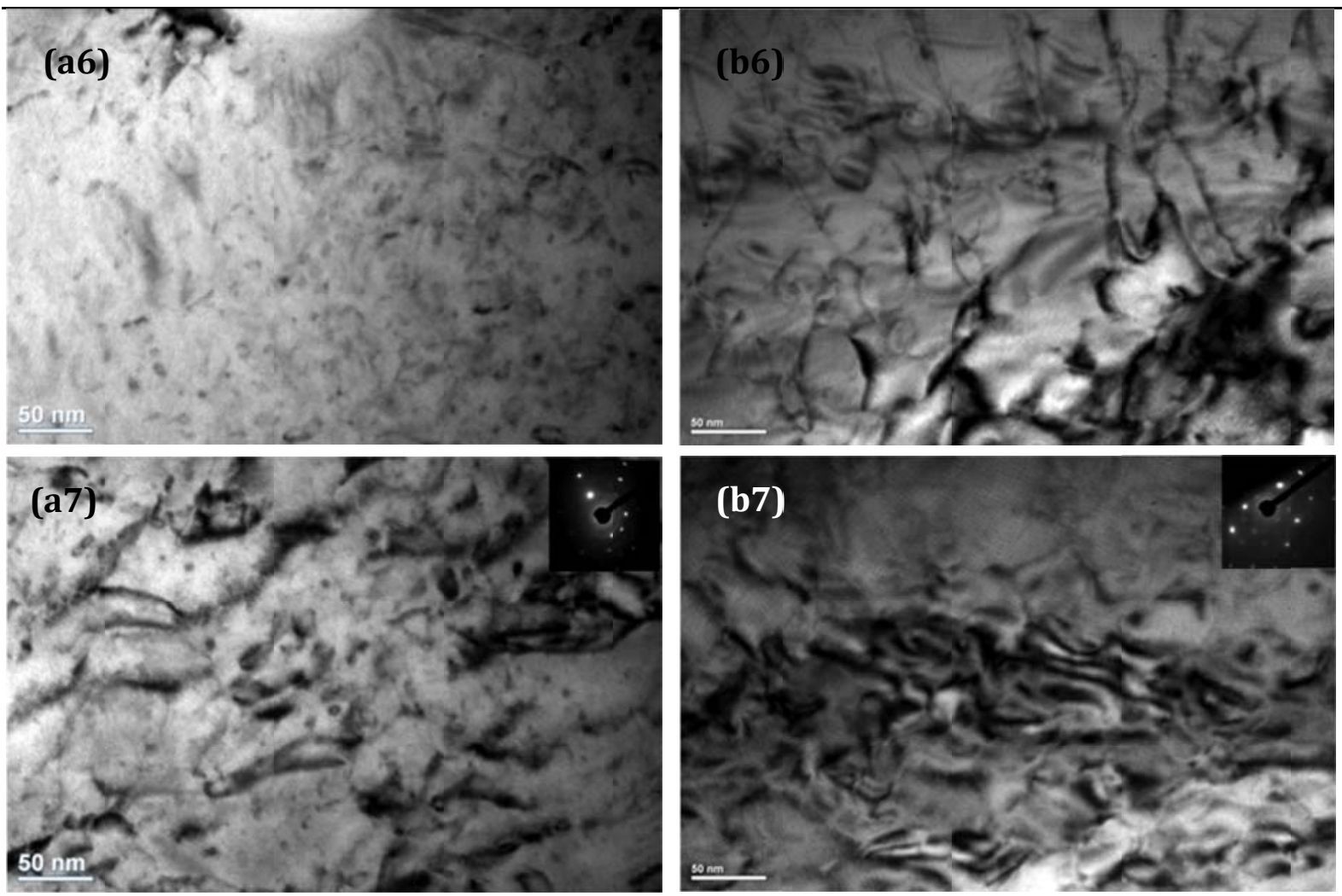

Figure 2.2 (cont.) Sequential change in the nucleation and growth of defect clusters in singe crystal $\mathrm{CeO}_{2}$ irradiated with $1 \mathrm{MeV} \mathrm{Kr}$ at $600^{\circ} \mathrm{C}$ (left column) and $800^{\circ} \mathrm{C}$ (right column) at various dose levels: (a6) and (b6) $3 \times 10^{15}$ ions $/ \mathrm{cm}^{2}$, and (a7) and (b7) $5 \times 10^{15}$ ions $/ \mathrm{cm}^{2}$. The observations were carried out from the [001] direction with $g=220$ reflection.

Figure 2.2 (a1) and (b1) show the as-annealed specimens at $600^{\circ} \mathrm{C}$ and $800^{\circ} \mathrm{C}$ respectively. A high density of black dots, indicated by arrows, is exhibited on both micrographs. These features were identified as dislocation loops induced during the Ar ion-milling process, and had the same appearance as the loops produced by $\mathrm{Kr}$ ion irradiation. Such polishing damage might be removed by annealing at a high temperature $\left(\sim 1400^{\circ} \mathrm{C}\right)[9-10]$. This step will be included in the future specimen preparation procedure. The density of black dots in figure 2.2 (a1) appears higher than in (b1), which is due to the different thicknesses of the observed areas.

As displayed in figure 2.2 (a2) - (a5) and (b2) - (b5), when the ion dose increases from $3 \times 10^{14}$ ions $/ \mathrm{cm}^{2}$ to $1 \times 10^{15}$ ions $/ \mathrm{cm}^{2}$, the average size of dislocation loops grows continuously: from 4.4 $\mathrm{nm}$ to $6.4 \mathrm{~nm}$ in diameter at $600^{\circ} \mathrm{C}$, and from $10 \mathrm{~nm}$ to $25.6 \mathrm{~nm}$ in diameter at $800^{\circ} \mathrm{C}$.

Figure 2.3 (a) depicts the comparison of average loop diameter during this irradiation process at $600^{\circ} \mathrm{C}$ and $800^{\circ} \mathrm{C}$. It is clearly seen that the loop size grows slightly at $600^{\circ} \mathrm{C}$, which is in contrast to the fast growth behavior at $800^{\circ} \mathrm{C}$. In addition, the average loop diameter at $600^{\circ} \mathrm{C}$ at the same dose level is much smaller than the one at $800^{\circ} \mathrm{C}$. For example, the loop size difference is $\sim 5$ times at $1 \times 10^{15}$ ions $/ \mathrm{cm}^{2}$.

Unlike the constantly increasing behavior of the loop size, the density of dislocation loops was found to increase at the beginning up to $8 \times 10^{14}$ ions $/ \mathrm{cm}^{2}$ at $600^{\circ} \mathrm{C}$, as shown in figure 2.3 (b), and then decrease at higher ion doses. The peak dose can be considered to correspond to the 
irradiation damage limit to sustain the maximum amount of individual loops. Before this dose, most of the radiation damage events contribute to the nucleation process of dislocation and dislocation loops. The formation and growth of dislocation loops at this stage is attributed to the accumulation of point defects. Beyond the peak dose, dislocation loops grow rapidly through coalescence/coarsening mechanisms. As displayed in figure 2.3 (b), the process of nucleation completed in an earlier stage at $800^{\circ} \mathrm{C}$, since the peak of density profile could have appeared before $3 \times 10^{14}$ ions $/ \mathrm{cm}^{2}$.

In order to understand the nature of the dislocation loops, Burgers vector determination was applied. However, the obtained information was not enough to specify the Burgers vectors: $<001\rangle,\langle 110\rangle$, or $\langle 111\rangle$. This is due to the limited tilting range $\left( \pm 40^{\circ}\right.$ in both of axes) around the single crystal orientation of [001]. In spite of the ambiguity, the analysis results helped to exclude the possibility of $b=\langle 110\rangle$. Helpful information was obtained from the past studies. Analogous dislocation loops were observed with $\mathrm{TEM}$ on ion-irradiated $\mathrm{UO}_{2}$, and their nature was characterized as interstitial type. Moreover, during $1000 \mathrm{keV}$ electron irradiation in $\mathrm{CeO}_{2}$ [7], defect clusters appeared as circular contrasts on bright-field TEM images. The $g \bullet b$ and trace analyses suggested the defect clusters were interstitial-type dislocation loops lying on $\{111\}$ planes with Burgers vector of $<111>$ direction.

The expanding of loop size leads to interaction of dislocation loops at higher doses, which started from the dose of $3 \times 10^{15}$ ions $/ \mathrm{cm}^{2}$ (figure 2.2 (a6)) at $600^{\circ} \mathrm{C}$ and $8 \times 10^{14}$ ions $/ \mathrm{cm}^{2}$ (figure 2.2 (b4)) at $800^{\circ} \mathrm{C}$. The dislocation loops then developed into line structure as shown in figure 2.2 (b6), and slowly aggregated to form dislocation networks by climb motion (figure 2.2 (b7)).

At $600^{\circ} \mathrm{C}$, the transition from individual loops to dislocation networks occurred in a much later stage. At the final dose of $5 \times 10^{15}$ ions $/ \mathrm{cm}^{2}$ (figure 2.2 (a7)), in addition to the similar dislocation line structure, small individual dislocation loops could still be distinguished.

There were no remarkable changes of defect microstructure observed in the investigated dose range once the tangled dislocation networks formed. Moreover, such inhomogeneous accumulations of dislocations are believed to serve as nuclei for recrystallization in high burnup nuclear fuel.

The diffraction patterns taken after irradiation at both $600^{\circ} \mathrm{C}$ and $800^{\circ} \mathrm{C}$ indicate the specimens remain perfectly crystalline (shown in the inset of figure 2.2 (a7) and (b7)). Hence, at the highest dose investigated, no amorphization or recrystallization of $\mathrm{CeO}_{2}$ occurred. 


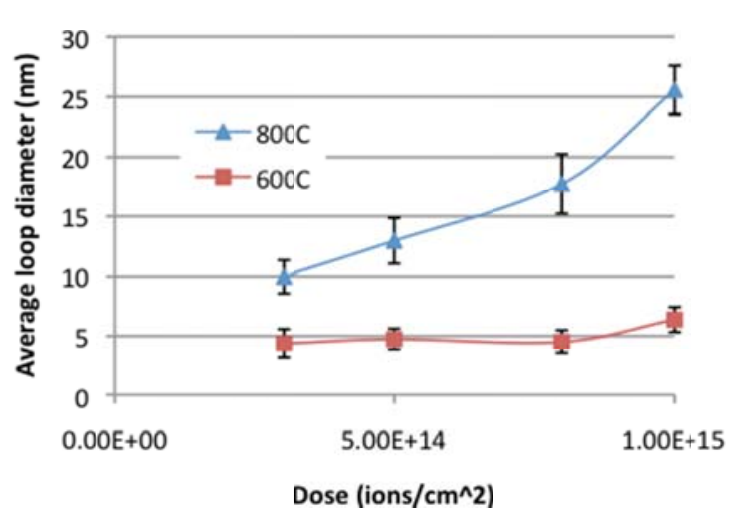

(a)

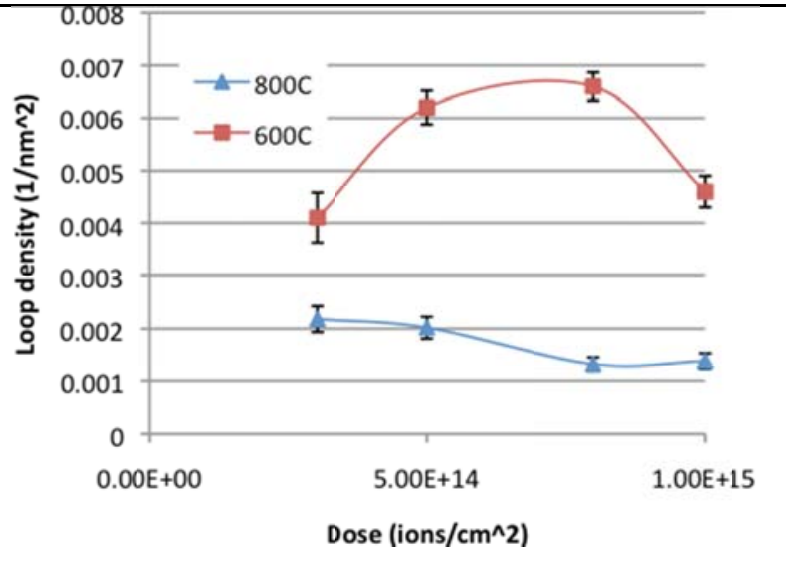

(b)

Figure 2.3. Variation of (a) the average diameter and (b) the density of dislocation loops as a function of ion dose during the irradiation of $1 \mathrm{MeV} \mathrm{Kr}$ ions at $600^{\circ} \mathrm{C}$ and $800^{\circ} \mathrm{C}$.

The substantial differences in damage ingrowth at irradiation temperatures of $600^{\circ} \mathrm{C}$ and $800^{\circ} \mathrm{C}$ were observed during irradiation of $1 \mathrm{MeV} \mathrm{Kr}$ ions, as presented in figure 2.2 and 2.3: much faster growth rate of defect structure at the higher temperature, and slight different defect morphology at the high doses. These facts may result from a combined effect of different temperature dependence of the accumulation rate of radiation damage and the mobility of defects (vacancies, interstitials and fission gas atoms) [11].

The temperature dependence of the kinetics of defect transformation in $\mathrm{UO}_{2}$ has been investigated through thermal recovery of defects in irradiated $\mathrm{UO}_{2}[9,12]$. U-vacancy mobility was accounted for the recovery stage centered around $870 \mathrm{~K}$, and the migration of U-selfinterstitials occurred in the temperature range $77-293 \mathrm{~K}$ in the $\mathrm{UO}_{2}$ irradiated with $1 \times 10^{15} \mathrm{Xe}$ ions $/ \mathrm{cm}^{2}$ at $5 \mathrm{~K}$ at $100 \mathrm{keV}$ energy.

Given the similarity in crystal properties, it is reasonable to deduce that the accelerated evolution process of defect structures at $800^{\circ} \mathrm{C}$ might be closely related to thermally activated Ce-vacancy mobility. At lower temperatures, Ce-interstitial mobility enables short-range migration of atoms [9] and is the governing factor in dislocation growth.

A chemical rate theory developed by Maehara [13] also correlated the growth behavior of interstitial-type dislocation loops with the mobility of vacancies [8]. At low temperatures where the vacancies are immobile, most of the interstitials recombine with the vacancies. At elevated temperatures, dislocation loops can grow quickly since migration efficiencies of interstitials and vacancies balance with each other at this condition.

Comprehensive TEM observations were performed to characterize the evolution of defect structures in single crystal $\mathrm{CeO}_{2}$ induced by $\mathrm{Kr}$ ion irradiation. The results are summarized as follows:

Evolution of microstructure in $\mathrm{CeO}_{2}$ was observed during in-situ irradiation of $1 \mathrm{MeV} \mathrm{Kr}$ ions at $600^{\circ} \mathrm{C}$ and $800^{\circ} \mathrm{C}$. Sequential TEM micrographs (figure 2.2) clearly illustrate the evolution process: the defects start to form clusters and loops at low dose, develop into extended dislocation lines and finally segregate together to form extremely tangled dislocation networks. 
In addition, the comparison of growth behavior of defect structures at $600^{\circ} \mathrm{C}$ and $800^{\circ} \mathrm{C}$ reveals the thermal activated Ce-vacancy mobility plays an important role in dislocation loop development.

\section{Gas Bubble Formation}

In order to investigate the precipitation of gas atoms in $\mathrm{CeO}_{2}$ crystal, $150 \mathrm{keV} \mathrm{Kr}$ ions were implanted into single crystal $\mathrm{CeO}_{2}$ TEM thin foils. At this low energy, most of gas atoms were stopped inside the specimens according to the SRIM [5] calculation, shown in table V.1.

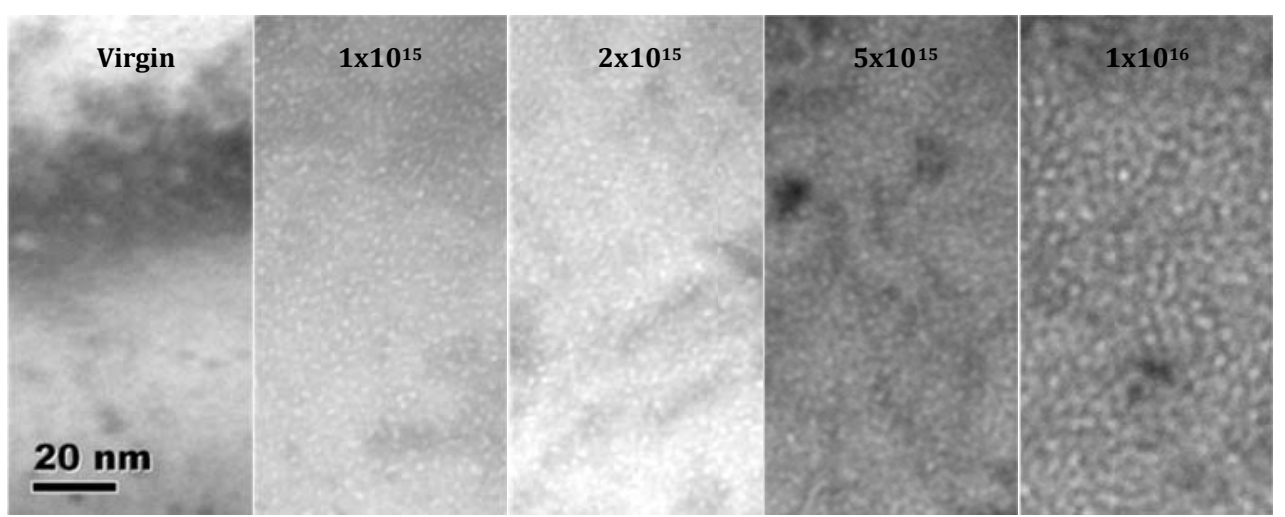

Figure 2.4. Bright-field images show sequential change of $\mathrm{Kr}$ bubbles with the increase of dose (ions $/ \mathrm{cm}^{2}$ ) at $600^{\circ} \mathrm{C}$. These micrographs are taken by underfocusing the objective lens.

Figure 2.4 shows the sequential change of $\mathrm{Kr}$ bubbles during implantation of $150 \mathrm{keV} \mathrm{Kr}$ ions at $600^{\circ} \mathrm{C}$. In these micrographs, $\mathrm{Kr}$ bubbles show as white dots with black edges (taken by underfocusing the objective lens). The size and density of $\mathrm{Kr}$ bubbles grow with the increase of dose. For the specimen irradiated to a dose of $1 \times 10^{16}$ ions $/ \mathrm{cm}^{2}$, the bubble density reaches 0.15 $\mathrm{nm}^{-2}$, and the average bubble diameter is $1.2 \mathrm{~nm}$. Similar features are observed on $\mathrm{UO}_{2}$ irradiated with $200 \mathrm{keV} \mathrm{Kr}$ at $600^{\circ} \mathrm{C}[14]$.

(a)

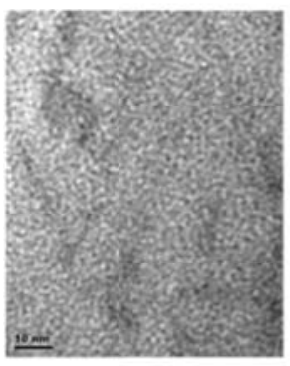

Underfocus

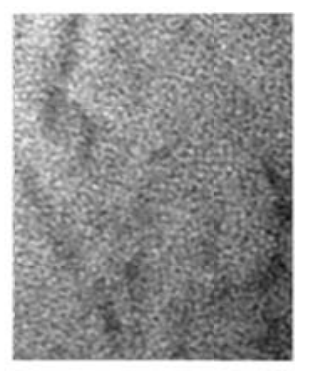

Overfocus

(b)

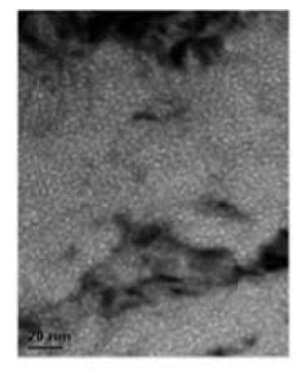

Underfocus

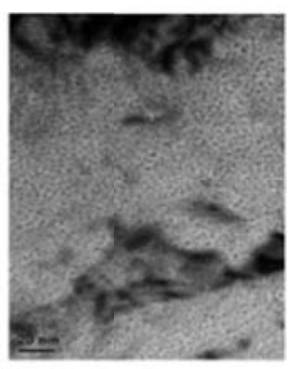

Overfocus

Figure 2.5 Bright-field TEM micrographs of single crystal $\mathrm{CeO}_{2}$ implanted with $150 \mathrm{keV} \mathrm{Kr}$ ions at a dose of $1 \times 10^{16}$ ions $/ \mathrm{cm}^{2}$ at (a) room temperature and (b) $600^{\circ} \mathrm{C}$. 
Figure 2.5 shows the bright-field TEM micrographs of in-situ irradiated $\mathrm{CeO}_{2}$ with $\mathrm{Kr}$ at 150 $\mathrm{keV}$ ion energy to a dose of $1 \times 10^{16}$ ions $/ \mathrm{cm}^{2}$. The peak $\mathrm{Kr}$ concentration is estimated to be $4.9 \%$ at this dose. Irradiation results at room temperature (figure $2.5(\mathrm{a})$ ) and $600^{\circ} \mathrm{C}$ (figure 2.5 (b)) are compared here. Typically, gas bubble features display opposite contrast at underfocus and overfocus conditions, as seen in figure 2.5 (b). However, such features were not found in figure 2.5 (a). In other words, gas bubbles did not form at room temperature. This fact might lead to the conclusion that a threshold temperature exist between room temperature and $600^{\circ} \mathrm{C}$ for $\mathrm{Kr}$ precipitation in $\mathrm{CeO}_{2}$ which is correlated with metal-vacancy mobility [14]. Another explanation of this phenomenon can be tied to the gas solubility limit dependent on temperature. The gas in solution needs to reach a critical value before a multi-atom nucleation process can occur. At higher temperatures, this dynamic solubility limit is reduced so that the nucleation occurs at a lower dose [15].

This result is confirmed by previous studies of gas atom precipitation conditions. Evans [14] reported that no visible bubbles were found below $400^{\circ} \mathrm{C}$ in irradiation of $200 \mathrm{keV} \mathrm{Kr}$ in $\mathrm{UO}_{2}$ for the dose of $5 \times 10^{15}$ ions/ $\mathrm{cm}^{2}$. More recently, Sabathier [9] found Xe bubbles in $\mathrm{UO}_{2}$ at $1 \times 10^{16}$ ions $/ \mathrm{cm}^{2}$ after annealing to $670 \mathrm{~K}$.

For the quantitative characterization, gas bubbles in figure 2.5 (b) were measured as $\sim 1.2 \mathrm{~nm}$ in average diameter and $1.5 \times 10^{17} \mathrm{~m}^{-2}$ for area density. These values are in good agreement with previously reported values $[9,14,16]$. Kashibe [16] observed the bubble size and density in low burnup spent fuel (23 MWd/kg) were $2 \mathrm{~nm}$ and $10^{24} \mathrm{~m}^{-3}$, which are comparable with present ion irradiation results. In addition, Xe bubbles which were $1.8 \pm 0.3 \mathrm{~nm}$ in size with a density of (4 \pm 2$) \times 10^{23} \mathrm{~m}^{-3}$ were found by Sabathier [9].

\section{Xenon Precipitation in $\mathrm{CeO}_{2}$}

$700 \mathrm{keV}$ Xe ion beams were obtained at the University of Illinois with a Van de Graaff accelerator. Ion beams hit the $\mathrm{CeO}_{2}$ crystals along [17] orientation. Irradiations were performed at room temperature and $600^{\circ} \mathrm{C}$. Three dose levels were achieved: $2 \times 10^{16}, 5 \times 10^{16}$ and $1 \times 10^{17}$ ions $/ \mathrm{cm}^{2}$, which result in peak dpa values of 106.3, 265.7 and 531.4 respectively, calculated with SRIM 2008 [5]. Following irradiation, TEM specimens were prepared by mechanically polishing followed by $3-5 \mathrm{kV}$ Ar ion milling. TEM observation of irradiation damage was preformed with JEOL 2010 $\mathrm{LaB}_{6}$ and JEOL 2200FS electron microscopes. The in-situ annealing experiment was conducted in the IVEM-Tandem facility at Argonne National Laboratory.

In the first series of experiments, the microstructures of irradiated $\mathrm{CeO}_{2}$ single crystals as a function of various dose levels are compared. As shown in figure 2.6, pure $\mathrm{CeO}_{2}$ was irradiated with $700 \mathrm{keV} \mathrm{Xe}$ at $600^{\circ} \mathrm{C}$ to varied dose levels. The thicknesses of thin films reduced during irradiation due to the sputtering effect. At the highest dose level, $1 \times 10^{17}$ ions $/ \mathrm{cm}^{2}$, the film thickness decreased $\sim 15 \mathrm{~nm}$. The inset diffraction patterns demonstrate that the specimens remain crystalline at $600^{\circ} \mathrm{C}$ even at a dose as high as $1 \times 10^{17}$ ions $/ \mathrm{cm}^{2}$, which confirms the high irradiation tolerance in fluorite-structure materials. Under similar irradiation conditions, Matzke [11 Bei thesis] reported a fully developed polygonization process in $\mathrm{UO}_{2}$ bombarded with 300 $\mathrm{keV} \mathrm{Xe}$ at $500^{\circ} \mathrm{C}$ in the range of $5-7 \times 10^{16}$ ions $/ \mathrm{cm}^{2}$ with the Rutherford backstattering (RBS) technique. In contrast to the results presented by Matzke [18], possible polygonization processes were not found in current TEM observations. 
Solid precipitates, which appear as black/black lobes, are visible in all three micrographs. Their average diameter grows from $\sim 1.9 \mathrm{~nm}$ in figure 2.6 (a) to $\sim 4.2 \mathrm{~nm}$ in figure 2.6 (c). These features were initially thought to be gas bubbles with a very high pressure or containing solid Xe precipitates. In a previous study, Nogita and Une [19] found solid Xe aggregated in spent fuel pellet with 45-83 Gwd/t. They observed Moiré fringes within the bubble area and extra spots in diffraction patterns, which indicates the existence of other solid particles in addition to the matrix $\mathrm{UO}_{2}$.

(a) $2 \times 10^{16}$ ions $/ \mathrm{cm}^{2}$

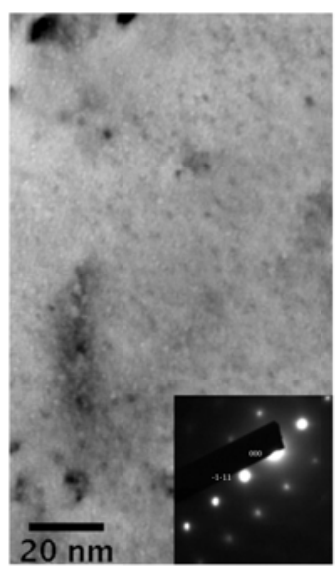

(b) $5 \times 10^{16}$ ions $/ \mathrm{cm}^{2}$

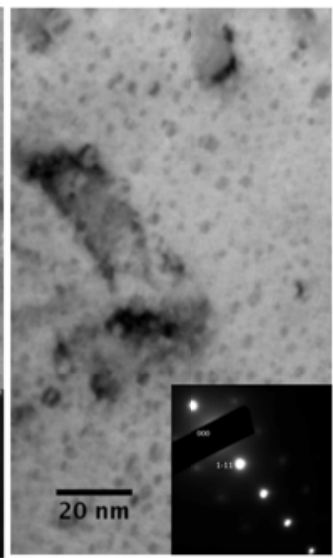

(c) $1 \times 10^{17}$ ions $/ \mathrm{cm}^{2}$

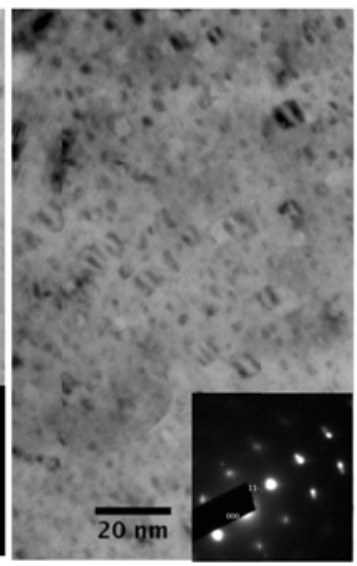

Figure 2.6 Cross-section bright-field TEM micrographs of pure $\mathrm{CeO}_{2}$ single crystals irradiated with $700 \mathrm{keV} \mathrm{Xe}$ at $600^{\circ} \mathrm{C}$. (a), (b) and (c) show the specimen irradiated to a dose of $2 \times 10^{16}$, $5 \times 10^{16}$ and $1 \times 10^{17}$ ions $/ \mathrm{cm}^{2}$ respectively. All images are taken with $g=\langle 1-11\rangle$. The electron beam is along the $<011>$ orientation.

However, there was no solid evidence obtained in this study that would lead to the same conclusion. Careful scanning TEM (STEM) analysis with JEOL 2200FS was performed. A darkfield Z-contrast STEM image of a precipitate area and its vicinity is displayed in figure 2.7 (a), along with its corresponding nano-beam diffraction pattern in figure 2.7 (b). In the faceted damaged area shown in figure 2.7 (a), the atomic lattices are slightly distorted, and the darker contrast suggests the absence of atomic layers or aggregation of a lower $\mathrm{Z}$ element. If the precipitates were highly condensed gas atoms, this area should be enriched with Xe. Nevertheless, there are no extra spots shown in figure 2.7 (b) and no Xe peaks detected with nano-area energy dispersive X-ray spectroscopy (EDX). These phenomena suggest another explanation for the nature of the precipitates as metallic Ce. The formation of Ce precipitates might be due to the active redox process in $\mathrm{CeO}_{2}$ at elevated temperatures and low oxygen pressures [20]. This is also supported by a subsequent annealing experiment. In this experiment, the specimen irradiated to $1 \times 10^{17}$ ions $/ \mathrm{cm}^{2}$ was placed in a TEM and heated with a heating stage so that the changes in microstructure could be monitored during annealing. The fringes in precipitated area disappeared at $600^{\circ} \mathrm{C}$, close to the melting point $\left(795^{\circ} \mathrm{C}\right)$ of metallic Ce, and reappeared after cooling back to room temperature. Although the irradiation was done at $600^{\circ} \mathrm{C}$, the microstructure of the specimen might change after taking it out from the accelerator and cooling down to the room temperature. The in situ annealing results shown in figure 2.8 demonstrate this possible change: the precipitates were in solid state at room temperature while became partial solid at $600^{\circ} \mathrm{C}$. Further investigation into the nature of the precipitates will be carried out with X-ray techniques. 
(a)

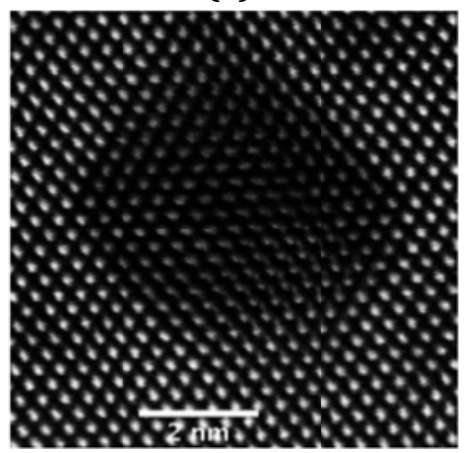

(b)

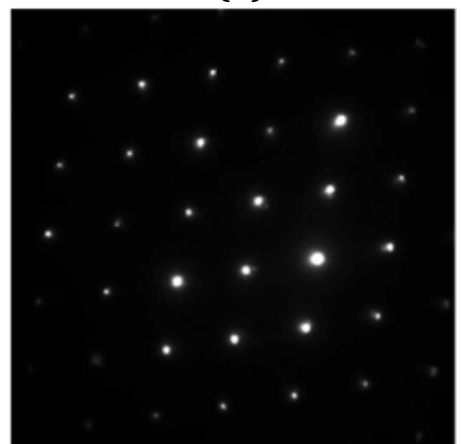

Figure 2.7 (a) Dark-field STEM micrograph of pure $\mathrm{CeO}_{2}$ irradiated at $600^{\circ} \mathrm{C}$ to a dose of $1 \times 10^{17}$ ions $/ \mathrm{cm}^{2}$. (b) Nano-beam diffraction pattern from the corresponding area.

The presence of the lanthanum dopant induces oxygen vacancy defects due to its alternate bonding nature. Given the chemistry of ceria doped with tri-valent dopants, the vacancies induced by La doping can help study the non-stoichiometric effects in ceria to the hypostoichiometric end, which resembles $(\mathrm{U}, \mathrm{Pu}) \mathrm{O}_{2}$ fuel. In addition, as one of the possible fission fragments, La can also help reveal the effect of fission fragments inside nuclear fuel material systems [21].

(a) Before annealing

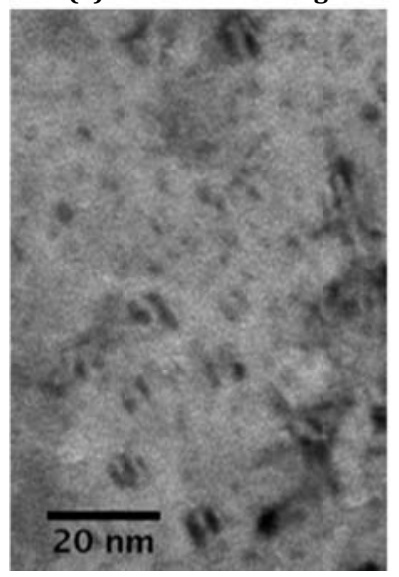

(b) $600^{\circ} \mathrm{C}$

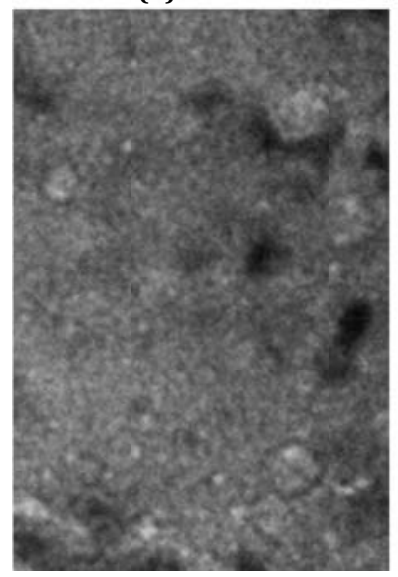

(c) After cooling

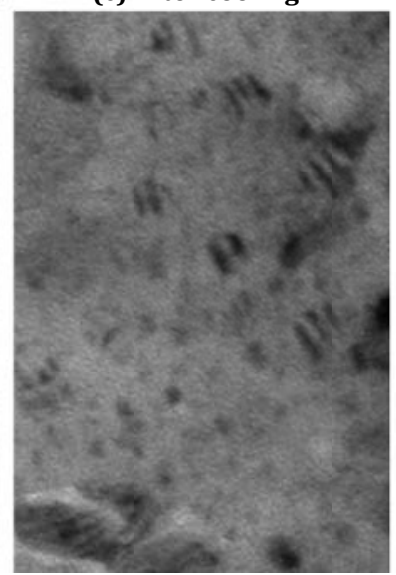

Figure 2.8 Sequential bright-field TEM micrographs during in-situ annealing of pure $\mathrm{CeO}_{2}$ irradiated at $600^{\circ} \mathrm{C}$ to a dose of $1 \times 10^{17}$ ions $/ \mathrm{cm}^{2}$. (a) microstructure of the specimen before annealing; (b) microstructure of the specimen heated at $600^{\circ} \mathrm{C}$; (c) microstructure of the specimen after cooling back to room temperature.

Figure 2.9 displays the through focus bright-field TEM micrographs taken on $\mathrm{CeO}_{2}$ doped with $5 \%$ La at $600^{\circ} \mathrm{C}$ to a dose of $1 \times 10^{17}$ ions $/ \mathrm{cm}^{2}$. Fresnel fringes on the edges of gas bubbles change from white to black with the objective lens adjusted from underfocus to overfocus conditions. On the infocus image, solid precipitates appear as round shaped features with fringes, which have a similar size and density as the ones shown in Figure 2.6 (c). However, such features were absent up to doses of $4 \times 10^{16}$ ions $/ \mathrm{cm}^{2}$, and were completely developed in the range of $4-10 \times 10^{16}$ ions $/ \mathrm{cm}^{2}$ for $600^{\circ} \mathrm{C}$ irradiation experiments. This delayed formation of precipitates compared to pure $\mathrm{CeO}_{2}$ might be attributed to lower xenon mobility and higher oxygen vacancy mobility in 
the doped materials [22]. Parallel experiments will be performed on $\mathrm{CeO}_{2}$ doped with $25 \% \mathrm{La}$, which should provide more information to help understand the La dopant effect.
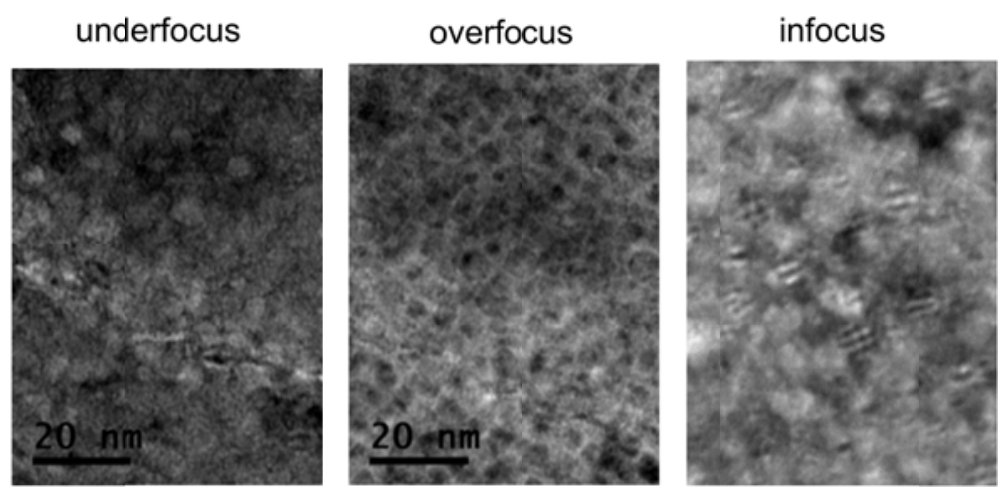

Figure 2.9 Plan view bright-field TEM micrographs on $\mathrm{CeO}_{2}$ doped with $5 \% \mathrm{La}$ at $600^{\circ} \mathrm{C}$ to a dose of $1 \times 10^{17}$ ions $/ \mathrm{cm}^{2}$. Electron beam is along $<001>$ orientation. All of the images were taken with the same magnification.

In irradiation experiments to very high doses, e.g. $1 \times 10^{17}$ ions $/ \mathrm{cm}^{2}$ at room temperature, there are no precipitates found in any of the materials studied. Thus, the formation of such precipitates must be a thermally activated process. There should be a threshold temperature for precipitate formation, existing between room temperature and $600^{\circ} \mathrm{C}$, which is due to the thermal activation of the oxygen vacancy mobility.

Xenon ion implantation in $\mathrm{CeO}_{2}$ and $\mathrm{Ce} / \mathrm{LaO}_{2}$ using $700 \mathrm{keV} \mathrm{Xe}$ ions were employed in this study to simulate irradiation processes in oxide fuel. A parametric study investigating the effects of ion fluence, irradiation temperature and La dopant concentration provide useful information for understanding the formation and growth processes of defect structures. Post-irradiation analyses were performed using ex-situ and in-situ TEM. Several important observations were made:

1) The materials remain crystalline after achieving very high doses $\left(1 \mathrm{x} 10^{17}\right.$ ions $\left./ \mathrm{cm}^{2}\right)$, which is due to the high irradiation tolerance of the fluorite crystal structure.

2) There was no evidence of grain subdivision or polygonization found. A possible explanation is that the thin film structure $(\sim 160 \mathrm{~nm}$ along ion beam direction), surface sputtering, and radiation-enhanced gas diffusion help relieve internal stresses accumulated during irradiation.

3) Solid precipitates appear in pure $\mathrm{CeO}_{2}$ at and above $2 \times 10^{16}$ ions $/ \mathrm{cm}^{2}$, but are absent in $\mathrm{CeO}_{2}$ doped with $5 \%$ La until the fluence exceeds $4 \times 10^{16} \mathrm{ions} / \mathrm{cm}^{2}$.

4) The nature of those precipitates is still being investigated using X-ray techniques. So far, the most probable interpretation is that Xe forms in the solid state in gas bubbles under very high internal pressure.

5) The formation of solid precipitates is a temperature dependent process. There is a threshold temperature existing between room temperature and $600^{\circ} \mathrm{C}$.

\section{B. Stoichiometry Effects}




\section{Evolution of Dislocation Structures: Low Does Regime}

In this section, the in situ experimental results are summarized in order to derive useful insights into the defect kinetics in the La doped $\mathrm{CeO} 2$ materials. It needs to be clarified that the in situ irradiation results are presented here in cropped images. The full-scale TEM images (mainly dark field images) are presented in the appendix A in order to provide more details on the experimental results so that readers can better appreciate the circumstances.

The following images in figure 2.10 show the results of in situ irradiation experiments in the form of dark field images with $220 \mathrm{~g}$ vector. The irradiation was conducted with $1 \mathrm{MeV} \mathrm{Kr}$ ion beam on the $5 \%$ La doped $\mathrm{CeO} 2$ planar view specimen at $600 \mathrm{C}$. Sequential dark field images of similar areas are selectively shown here to reflect a general trend. Areas are not exactly the same due to specimen shifting, inaccuracies in cropping and so on, but the images should be very representative. More detailed discussions are provided in the followings.
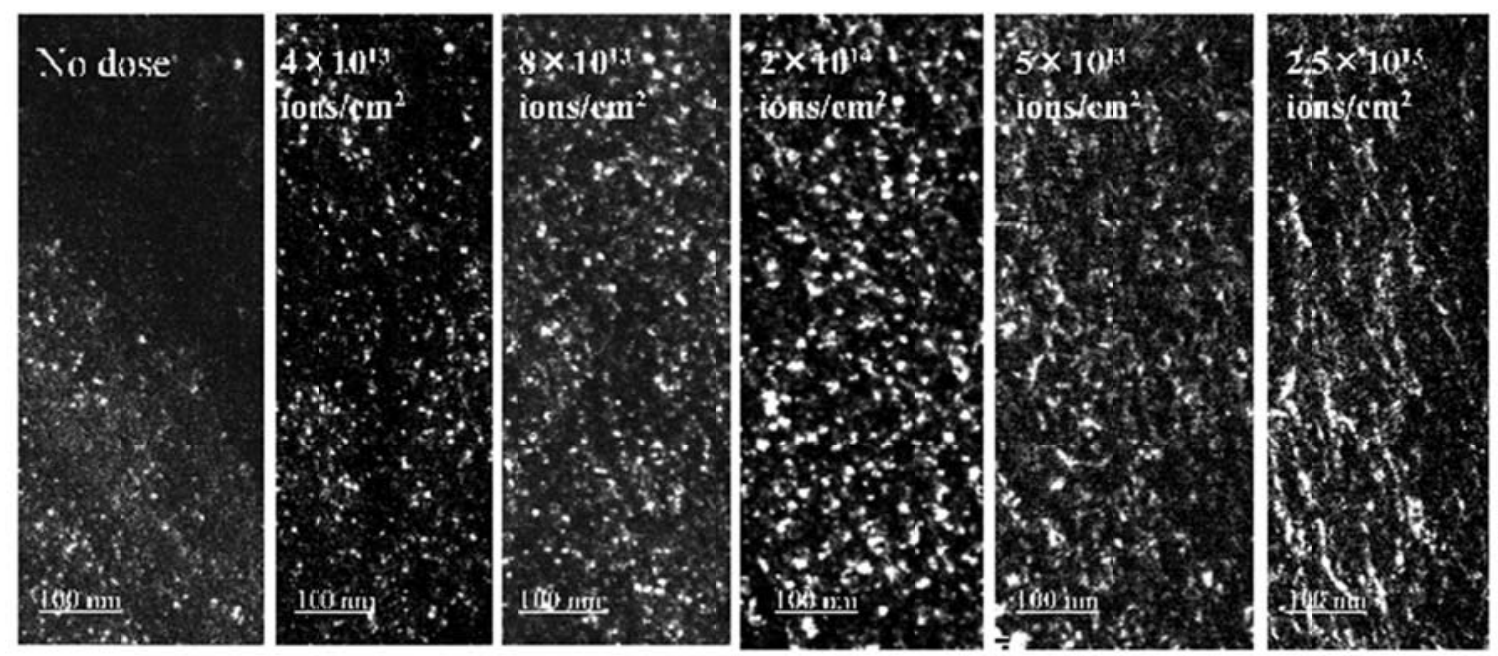

2.10 In situ $1 \mathrm{MeV}$ kr irradiation at 600C carried out on 5\% La doped Ce02 plan view specimen sequential dark field images with $\mathrm{g}=220$ : (from left to right) no dose, $4 \times 10^{13}$ ions $/ \mathrm{cm}^{2}, 8 \times 10^{13}$ ions $/ \mathrm{cm}^{2}, 2 \times 10^{14}$ ions $/ \mathrm{cm}^{2}, 5 \times 10^{14}$ ions $/ \mathrm{cm}^{2}$ and $2.5 \times 10^{15}$ ions $/ \mathrm{cm}^{2}$

Both qualitative and quantitative information are extracted from this set of images. The qualitative information involves mainly the evolution of defect structures, while the quantitative information concerns the density of such defects.

Due to the initial drift of the specimen at the beginning of the irradiation, it was very difficult to keep the view area the same during the entire experiment. However, a very distinctive marker (a big piece of contamination) was found to help track the area. This marker does not change its shape or contrast with changing diffraction conditions. With the documentations of bright field images, it was determined that all the images (except the ones taken in the very end) were taken in nearly the exact same area. This is extremely helpful to make sure that the quantitative data measured from different images have a strong level of comparability.

It can be clearly seen that the density of the white dot defect structures (which are believed to be dislocation loops) increases significantly with increasing dose. During the in situ experiment at the lower dose range (dose less than or equal to $2 \times 10^{14}$ ions $/ \mathrm{cm}^{2}$ ), two types of events can be clearly observed: the emergence of white dot defect structures, and the disappearance of such 
structures. These two events are interpreted as the formation of dislocation loops by irradiationinduced displacements and possible annihilation of dislocation loops or dislocation loops changing its burgers vector so that it satisfies the invisible criteria. The former mechanism of annihilation is believed to be the more plausible explanation.

At the dose level of $2 \times 10^{14}$ ions $/ \mathrm{cm}^{2}$ or higher, dislocation loop type of defect structures are seen to interact with each other to start to form more extended defect structures - possibly segment dislocations. Qualitatively, the density of the dislocation loop structures decreases while the density of the more extended defect structures pick up. At the intermediate dose of this experiment, $1 \times 10^{15}$ ions $/ \mathrm{cm}^{2}$, the density of the dislocations become so high that individual dislocation loop structures seen earlier can hardly be identified any more.
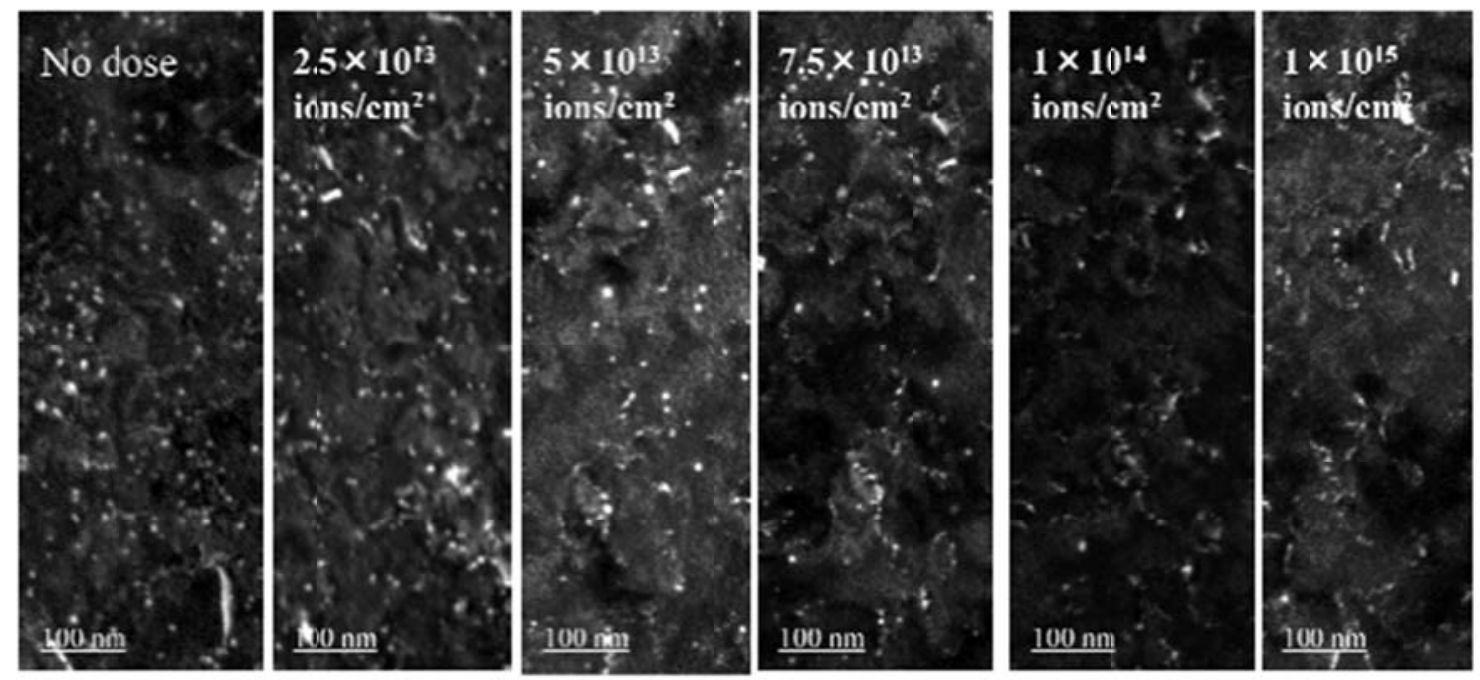

$2.11 \mathrm{In}$ situ $1 \mathrm{MeV} \mathrm{Kr}$ irradiation at 600C carried out on 25\% La doped Ce02 plan view specimen - sequential dark field images with $\mathrm{g}=220$ : (from left to right) $2.5 \times 10^{13}$ ions $/ \mathrm{cm}^{2}, 5 \times 10^{13}$ ions $/ \mathrm{cm}^{2}, 7.5 \times 10^{13}$ ions $/ \mathrm{cm}^{2}, 1 \times 10^{14}$ ions $/ \mathrm{cm}^{2}, 2 \times 10^{14}$ ions $/ \mathrm{cm}^{2}, 1 \times 10^{15}$ ions $/ \mathrm{cm}^{2}$

The images from figure 2.11 demonstrate sequential dark field TEM images from the in situ experiment $1 \mathrm{MeV}$ doubly charged $\mathrm{Kr}$ ion beam irradiation. The irradiation was carried out at $600 \mathrm{C}$ on $25 \%$ La doped $\mathrm{CeO}_{2}$ specimen. The images were taken with a consistent g vector of 220 . Weak beam conditions were applied in order to show the structure evolution of the defect/defect clusters.

Again, due to the initial drift of the specimen at the beginning of the irradiation, it was very difficult to keep the view area the same during the entire experiment. However, a very distinctive marker was found to help track the area. This marker does not change its shape or contrast with changing diffraction conditions. The diffraction conditions for the sequential TEM images were kept very similar throughout the course of the experiment.

It is very interesting to see that the density of the white dot defect structures (which are believed to be dislocation loops) decreases significantly with increasing dose. At the same time the individual defect structures disappear, small extended defect structures pre-existed in the specimen can be seen to grow in size. This growth, however, was not significant. There are also defect structures forming as dislocation loops close to each other joined together. The formation of defect structures following this mechanism, however, is rather infrequent compared to the 
irradiations carried out on $5 \%$ La doped $\mathrm{CeO} 2$ at the same temperature of $600 \mathrm{C}$. This demonstrates a very interesting effect of Lanthanum as impurity. A plausible (but not definitive) explanation is that the higher initial density of oxygen vacancy $(12.5 \%$ compared to $2.5 \%$ between two materials) affected the defect chemistry that the dislocation loops react with the readily available oxygen vacancies and annihilate. Also, the presence of Lanthanum in the lattice effectively decreases the mobility of the dislocation loops. A combination of the two effects led to the trends observed experimentally. There are two hypotheses associated with the above provided explanation: the dislocation loops are primarily of oxygen interstitial type (that's why they annihilate with oxygen vacancies); the mobility reduced by the presence of Lanthanum couldn't be caused by the higher concentration of oxygen vacancies alone. The first hypothesis has been tentatively confirmed by the burgers vector analyses combined with the inside/outside contrast analyses earlier. However, due to the unclear natures of the outside contrast of the dislocation loops and the fact that the inside/outside contrast experiment is very complicated (one small mistake will result in totally opposite conclusions), the inside/outside experimental results are not shown in this dissertation and are not held as supporting evidence to the speculation on the nature of the dislocation loops. Other supporting evidences will be provided in the later chapter where the void/bubble mobility is analyzed. The second hypothesis was tested by the kinetic Monte Carlo simulation together with the lattice simulation. The results will be shown in the chapter for modeling later as well.

The following figures 2.12 to 2.13 summarize results on quantitative measurements for the dislocation loop density and sizes. For the density measurements, five boxes with same dimension of $220 \mathrm{~nm}$ by $220 \mathrm{~nm}$ were drawn and the dislocation loops that fall within these boxes were counted. As a result, the density values are statistical averages. For the size measurements of the loops, 100 loops have been surveyed. It becomes a little trickier for the measurements on the size of the dislocation loops as the measurements are done not really on the dislocation loops but on their diffraction contrasts. The loops could exhibit inside or outside contrast and the shape of the loops change according to specific tilting conditions. Therefore, careful planning was done before the experiments such that the measurements were taken out on similar locations on the Kikuchi map (indicating very similar tilting). The g vectors were set up right so the features exhibiting diffraction contrasts measured in the images all represent the inside contrasts of the dislocation loops. This implies that the resulting size values for all experiments were in fact the length of the inside contrast of dislocation loops. The realistic size (on the long side) would be bigger than the measured values. Under these very similar tilting conditions, all the loops measured exhibited elliptical shapes with a long side to short side ratio of about 1.8 2. It is therefore believed that the measurements provide consistent data between each data point on the various dose levels. The long sides of the dislocation loops were measured as it was easier and more accurate to measure the long sides when the loops were of such small sizes. 


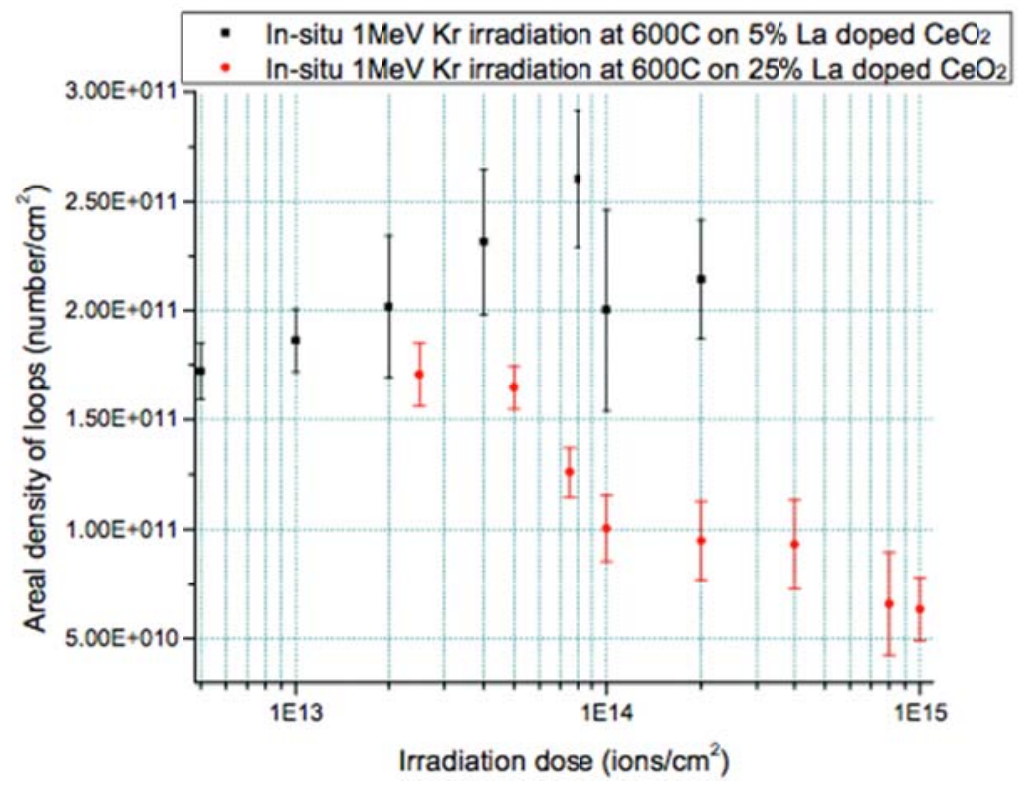

Figure 2.12 Evolution of number density of dislocation loops for in situ $1 \mathrm{MeV} \mathrm{Kr}$ irradiations at 600C

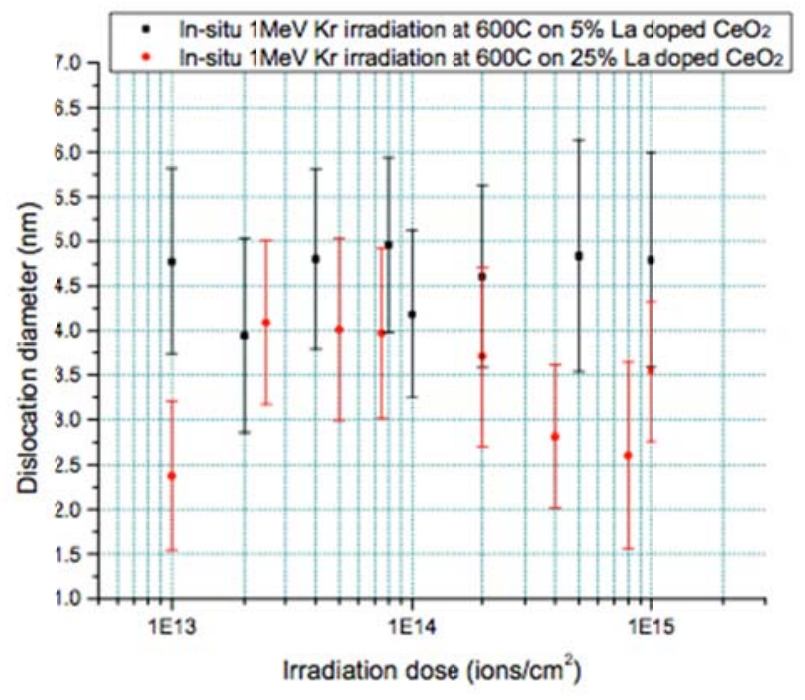

2.13 Evolution of size of dislocation loops for in situ $1 \mathrm{MeV}$ Kr irradiations at 600C

\section{Gas Bubble Formation: High Dose Regime}

When the dose level of the ion beam irradiation goes up to the high dose regime (high dose regime in this study refers to a dose level of no less than to $5 \times 10^{15}$ ions $/ \mathrm{cm}^{2}$ ), the dislocation network becomes highly concentrated (with a high number density of dislocations tangled together). The diffraction contrast becomes worse and worse for quantitative and even for the qualitative measurements of such features in the material lattice due to the buildup of defects and complications of the local geometry of the specimen (more bending and deteriorated surface 
conditions). With the observation made in the earlier chapters that defect of contrary natures (interstitial and vacancy) tend to cluster, the buildup of the dislocation network can be interpreted as a result of the clustering of higher and higher concentration of interstitial type defects produced by the ion beam irradiation. At the same time when interstitial type defects become more concentrated and cluster, vacancy type defects are believed to cluster in their own ways - they start to form voids. As voids form in the material lattice, they become ideal trapping sites for inert gas. When a high concentration of inert gas atoms is deposited in the lattice, bubbles are believed to form. This is only a quick glance at the interpretation from a theoretical perspective.

The experimental evidence of void/bubble growth is presented and discussed. The underlying mechanisms of such growth are derived from the experimental data. Here, the general guidelines towards KMC level modeling are provided based on the observation and analyses of qualitative trends and quantitative results obtained by the experiments.

For all the experiments conducted, the size distributions and the density of void/bubble structures have been measured and analyzed. The procedures for the measurements are clarified as follows.

For the density measurements, five boxes of dimension $32.5 \mathrm{~nm} \times 32.5 \mathrm{~nm}$ are drawn with the number of features registered with the reversed Fresnel contrast in under focusing and over focusing conditions counted. The resulting number density is therefore a statistical average of the five individual measurements. The tricky part of this process is the selection of the areas to be measured. It is ideal to measure the areas with consistent thickness. However, diffraction contrasts often limit the areas available for good measurements. It then becomes a quest for balance to find areas with good contrast (minimum diffraction contrast) and similar thicknesses.

For the size measurements, 120 features that exhibit reversed Fresnel contrast in under and over focusing conditions have been measured for each single data point in order to give reasonable statistical survey data rather than to have small measured quantity induced bigger statistical errors. The area of the void/bubble features was measured using the software package Image $J$ and the diameter is then derived from the measured area. The resulting diameter in units of $\mathrm{nm}$ is then a statistical average of the survey of 120 features. The large error bars are then believed to be mostly due to the wide size distributions of features recorded in the data rather than a result of under measurement. The size distributions are also given as key results obtained from these measurements.

Figure 2.14 and figure 2.15 show respectively void areal number density dependence, void diameter dependence and void volume dependence on the irradiation dose for $1 \mathrm{MeV} \mathrm{Kr}$ ion irradiations at $600 \mathrm{C}$ in $5 \%$ La doped $\mathrm{CeO}_{2}$. The black data set shows the results of ex situ irradiation experiments and the red data set shows the results of the in situ irradiation experiments. Unfortunately, the quality of the data from in situ experiment on void size and number density is not good enough for quantitative analyses (due to the quality of under-focus and over-focus images being low with objective astigmation problems mainly). As a result, only the data point for the final dose $\left(1 \times 10^{16}\right.$ ions $\left./ \mathrm{cm}^{2}\right)$, for which the measurement was done with the 2011 LaB6 facility in MRL at UIUC, is presented. The number density was measured with a $32.5 \mathrm{~nm} \times 32.5 \mathrm{~nm}$ square window at different locations on an image (with likewise thickness). The obtained data were then statistically averaged. The data points and associated error bars reflect the means and standard deviations of the measurements. The measurements were all 
done in regions with comparable thicknesses so that data from different specimens could be compared in a meaningful way.

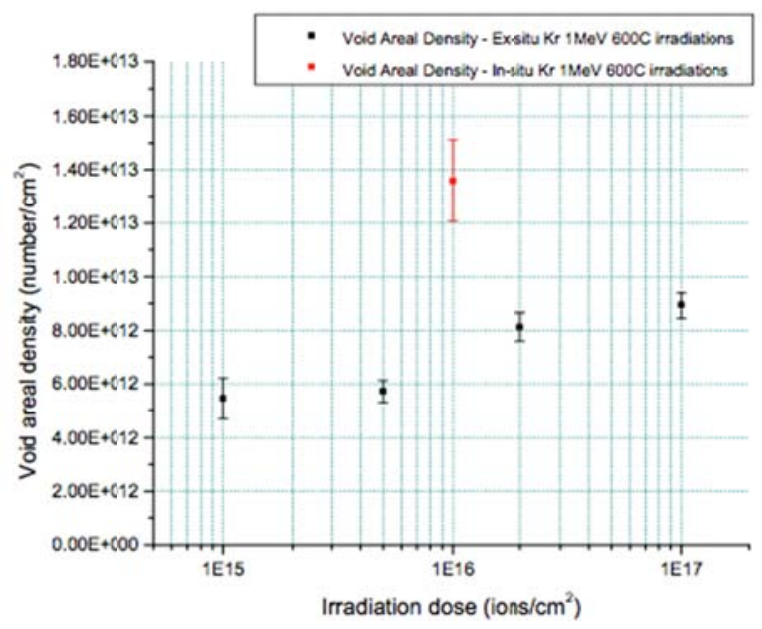

Figure 2.14 Void areal density dependence on irradiation dose for $1 \mathrm{MeV} 600 \mathrm{C} \mathrm{kr}$ irradiations on $5 \%$ La doped $\mathrm{CeO}_{2}$

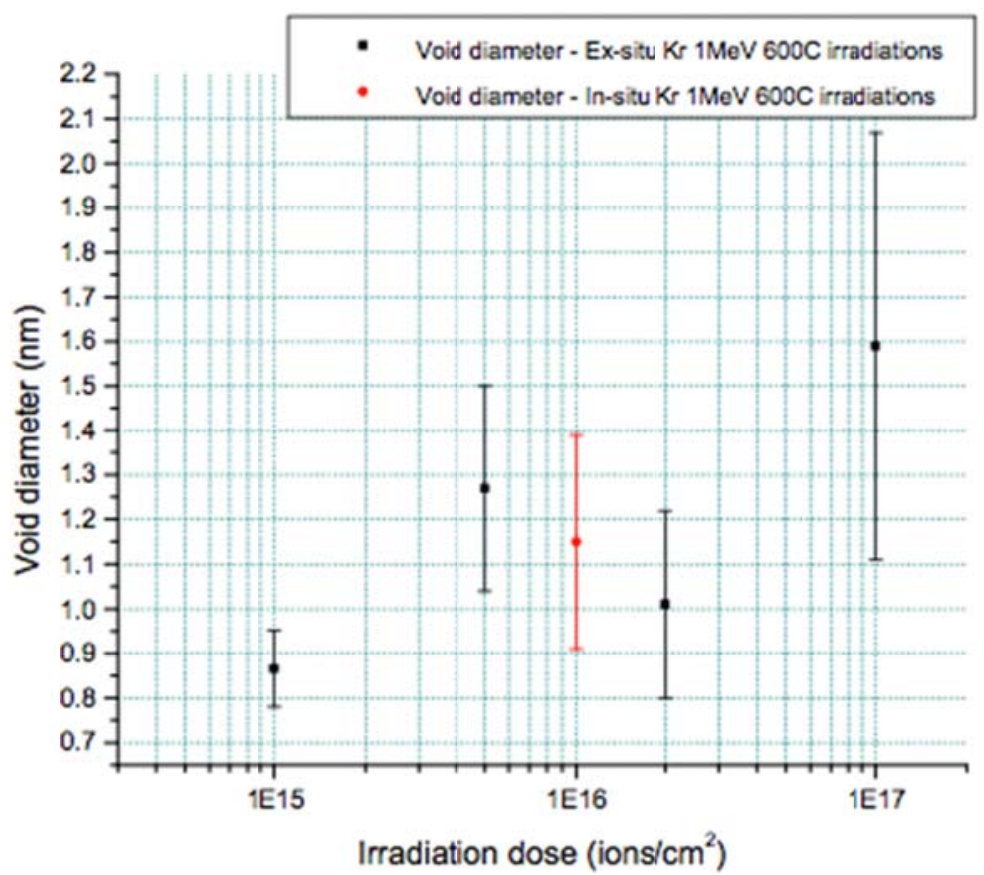

Figure 2.15 Void diameter dependence on irradiation dose for $1 \mathrm{MeV} 600 \mathrm{C} \mathrm{kr}$ irradiations on 5\% La doped $\mathrm{CeO}_{2}$

Overall, the in situ experiment seems to yield higher number density of voids with similar void size. Seems is used here because the error bars of data points overlap. However, because the main sources of the error bars are believed to come from the variations in the sizes of the voids, the higher number density is believed to exist in the in situ experiment specimen. As a result, the void volume is higher for the in situ experiment. The explanation of this difference is twofold. 
Firstly, the free surfaces readily available (due to relatively thin specimen) give rise to higher mobility of defect species as there is essentially no bulk material in the region of interest and therefore the concentration gradient serves as a driving force to move defects. Secondly, due to the same fact that the specimen is relatively thin, sputtering yield is much higher in the in situ experiments than ex situ experiments.

A general trend of increasing number density and increasing void size can also be seen from the ex situ irradiation results. As a result, the void volume also shows a rising trend. The error bar is much bigger for the case of the dose level of $1 \times 10^{17}$ ions $/ \mathrm{cm}^{2}$ as the size of the voids varies a lot (from $1.2 \mathrm{~nm}$ in diameter to $3 \mathrm{~nm}$ in diameter). The proof of this assertion can be seen later where the size distribution profiles of void structures are shown.

It is interesting to notice that the number density of voids increases at a much slower rate compared to the dose increase. An increasing trend on the measured average void diameter can be clearly observed. This suggests that coalescence occurs in the material as the irradiation dose increases. If there is absolutely no coalescence and voids created are purely a reflection of ion beam displacing atoms, the number density of voids should be almost linear. A near linear trend in the data shown by linear-log scale indicates a much slower growth. The event based model is therefore not solid. In the rate theory related literatures [60 Di thesis], a crude square root law was suggested for coalescence based growth. This is much more likely in the results shown.

Figure 2.15 and figure 2.16 show respectively the comparison of void areal number density, void diameter and void volume with $1 \mathrm{MeV} \mathrm{Kr}$ irradiations at $600 \mathrm{C}$ between $5 \%$ La doped $\mathrm{CeO} 2$ and $25 \%$ La doped $\mathrm{CeO} 2$. The black data set shows the results of ex situ irradiation experiments on $5 \%$ La doped $\mathrm{CeO} 2$. The red data set shows the results of ex situ irradiation experiments on $25 \%$ La doped $\mathrm{CeO} 2$. The blue data point shows in situ experimental results for $5 \%$ La doped $\mathrm{CeO} 2$ simply for comparison. The measurements were all done in regions with comparable thicknesses so that data from different specimens can be compared in a meaningful way.

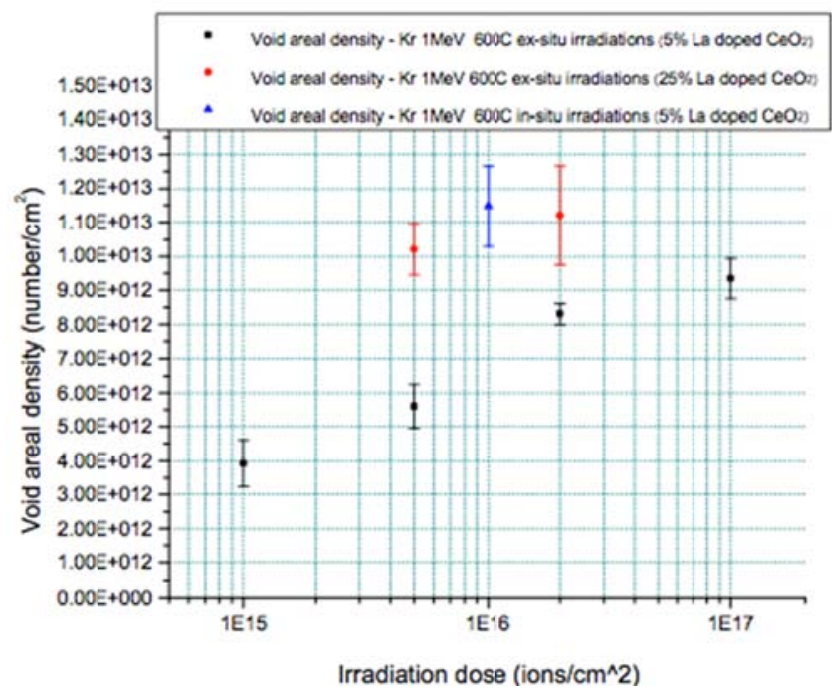

Figure 2.15. Comparison of void areal number density between $1 \mathrm{MeV} \mathrm{Kr}$ irradiation at $600 \mathrm{C}$ on $5 \%$ La doped $\mathrm{CeO}_{2}$ and $25 \%$ doped $\mathrm{CeO}_{2}$ 


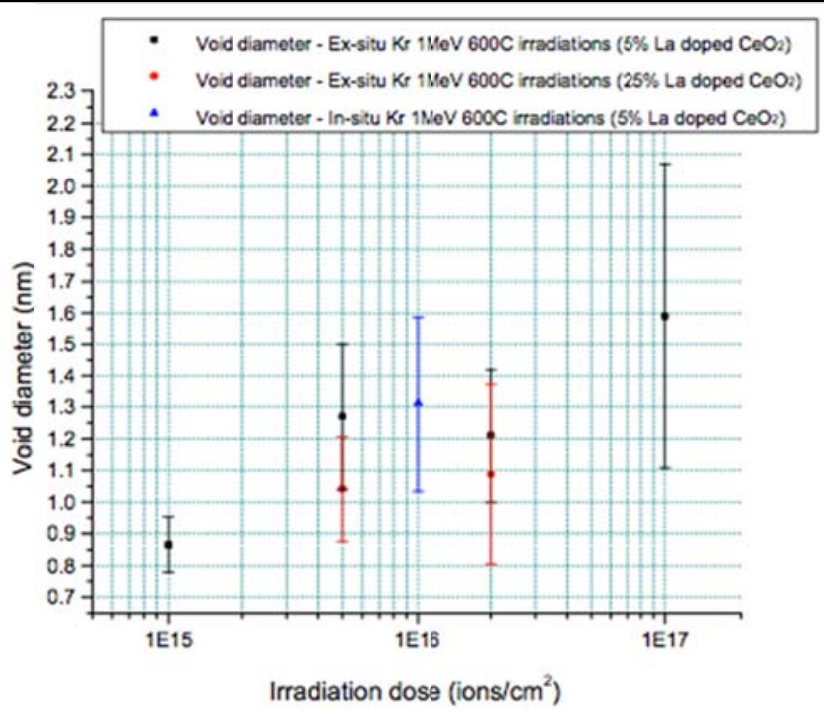

Figure 2.16. Comparison of void diameter between $1 \mathrm{MeV}$ Kr irradiation at 600C on 5\% La doped $\mathrm{CeO} 2$ and $25 \%$ La doped $\mathrm{CeO}_{2}$

It is then interesting to compare the void size distributions between results from irradiations on $5 \% \mathrm{La}$ doped $\mathrm{CeO} 2$ and results from irradiations on $25 \% \mathrm{La}$ doped $\mathrm{CeO} 2$ with a corresponding dose level. The following figures 2.17 presents such comparisons. A rather clear insight can be drawn from these comparisons that the voids are consistently smaller, but that they are present with much higher number densities in the irradiations carried out on $25 \% \mathrm{La}$ doped CeO2. Decreased coalescence is therefore determined to have caused the dramatic differences. The underlying mechanism to cause less coalescence deserves more in-depth discussions here.

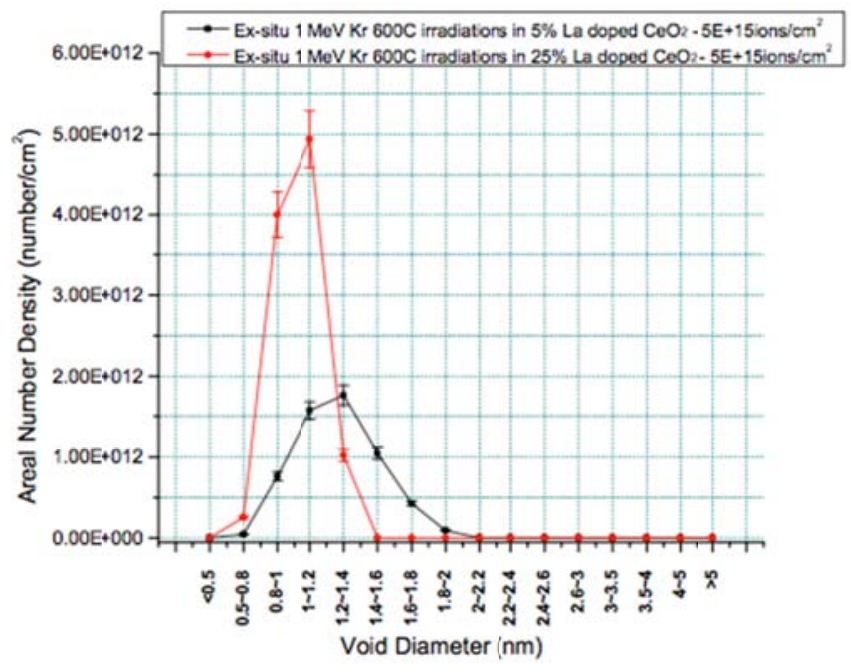

Figure 2.17. Comparison between void size distribution of $5 \%$ La doped $\mathrm{CeO}_{2}$ and $25 \%$ La doped $\mathrm{CeO}_{2}\left(1 \mathrm{MeV} \mathrm{Kr}\right.$ irradiations at $600 \mathrm{C}$ to a same dose of $5 \times 10^{15}$ ions $/ \mathrm{cm}^{2}$ )(lines are drawn to guide the eyes)

It is useful to reflect on the past work by J. Faber et al. [23]. The dc conductivity reflects the 
oxygen vacancy diffusivity as the mechanism of charge transfer inside this material is believed to be via an oxygen vacancy mechanism [24]. As a result, the curve of dc conductivity versus dopant concentration in [23] reflects oxygen vacancy self-diffusivity change with La dopant concentration within the $\mathrm{CeO} 2$ lattice. As discussed earlier, $5 \%$ La concentration was chosen to ensure the close to maximum oxygen vacancy diffusivity and $25 \%$ La concentration was chosen to investigate the change on defect mobility given this result that at $25 \%$ La concentration the dc conductivity becomes significantly lower (almost 2 orders of magnitude). The activation energy for oxygen vacancy is also shown in reference [23], where the smallest value in the activation energy in La doped $\mathrm{CeO} 2$ occurs at the concentration of about 1 atom\%, which is inconsistent with the diffusivity data as the pre-exponential factor is also dependent on the La composition. But in general, the 5 atom\% La concentration provides an activation energy value close to the minimum value and the 25 atom\% La concentration provides a much higher activation energy (about $1.1 \mathrm{eV}$ as opposed to about $0.75 \mathrm{eV}$ ). This is also confirmed by the KMC simulation done in this work which will be shown later.

Another very important finding is illustrated in the following figure 2.18. Fresnel fringes that would appear when different material lattices overlap can be clearly identified in this bright field TEM image at about in focus. These structures can be seen from earlier TEM images with under/over focusing conditions. However, they are most clearly seen when the focusing condition is near in focus and when the specimen is tilted to be close to the zone axis. The structures are believed to be solid state Xe precipitates as they are represented by some extra spots in the SAD diffraction patterns. The SAD diffraction pattern is shown in figure 2.19. The extra diffraction spots correspond to an f.c.c. type atomic structure with a lattice constant of about $6.0 \mathrm{~nm}$. This is the form that most Xe solid state precipitates have been observed [25-26]. Figure 2.20 (a) and (b) illustrate the High Resolution TEM (HRTEM) image of such faceted solid state Xe precipitate structure and the corresponding Fast Fourier Transform (FFT) in order to show the different facets of the precipitate structure. It can be seen that the structure has both (200) and (220) type of facet planes. Further experiments near the [011] zone will be needed to illustrate the three dimensional structure of such precipitate.

The discovery of the faceted solid state Xe precipitates shows an important factor that simulations work carried out later will need to account for. The factor is the solution of Xe inside the $\mathrm{CeO}_{2}$ and $\mathrm{La}$ doped $\mathrm{CeO}_{2}$ material lattice or pure $\mathrm{CeO}_{2}$ lattice. It suggests a possible competing mechanism at high dose range of the irradiations. This is to say that when the Xe concentration in the material lattice becomes very high, Xe tends to stay either in a bubble form or precipitate from the lattice. The Xe atoms in solution with the material lattice can migrate to a bubble structure and get absorbed into such structures, or they can migrate to become associated with other Xe atoms in solution and precipitate as solid state Xe precipitates when there are enough Xe atoms available. A very crude estimate indicates that there are about 1,400 Xe atoms in one of such precipitate structure. The formation of such a structure then requires a huge concentration of Xe coming close enough to each other. It can therefore be speculated that room temperature experiments might not produce these structures simply due to the low mobility of Xe. In other words, such precipitates might only be expected in the lattice at room temperature when the random injection of Xe by implantation can gather some 1,400 Xe atoms in a solid state lattice structure. The dose can then be expected to be extremely high. As $25 \% \mathrm{La}$ doped $\mathrm{CeO}_{2}$ has much lower Xe mobility as discussed earlier, a same dose experiment on that material could be very informative.

Interestingly, similar precipitates have not been found in room temperature experiments on 
either 5\% La doped $\mathrm{CeO}_{2}$ and 25\% La doped $\mathrm{CeO}_{2}$ at rather similar dose level. At 600C, a lower dose level such as $4 \times 10^{16}$ ions $/ \mathrm{cm}^{2}$ clearly does not produce such solid state Xe precipitates. The precipitates were, however, found in pure $\mathrm{CeO}_{2}$ (from Bei Ye's experimental results on pure $\mathrm{CeO}_{2}$ ) irradiated with $700 \mathrm{keV} \mathrm{Xe}$ at a much wider dose range (from $1 \times 10^{17}$ ions/ $\mathrm{cm}^{2}$ down to 2 $\mathrm{x} 1016$ ions $/ \mathrm{cm}^{2}$ ), but only at $600 \mathrm{C}$, not the room temperature experiments. This could suggest that the formation of such Xe precipitate is a thermally activated process. Further investigations need to be done to explain the dose dependence of the formation of solid state Xe precipitates on different materials (pure $\mathrm{CeO}_{2}, 5 \%$ La doped $\mathrm{CeO}_{2}$ and 25\% La doped $\mathrm{CeO}_{2}$ ).

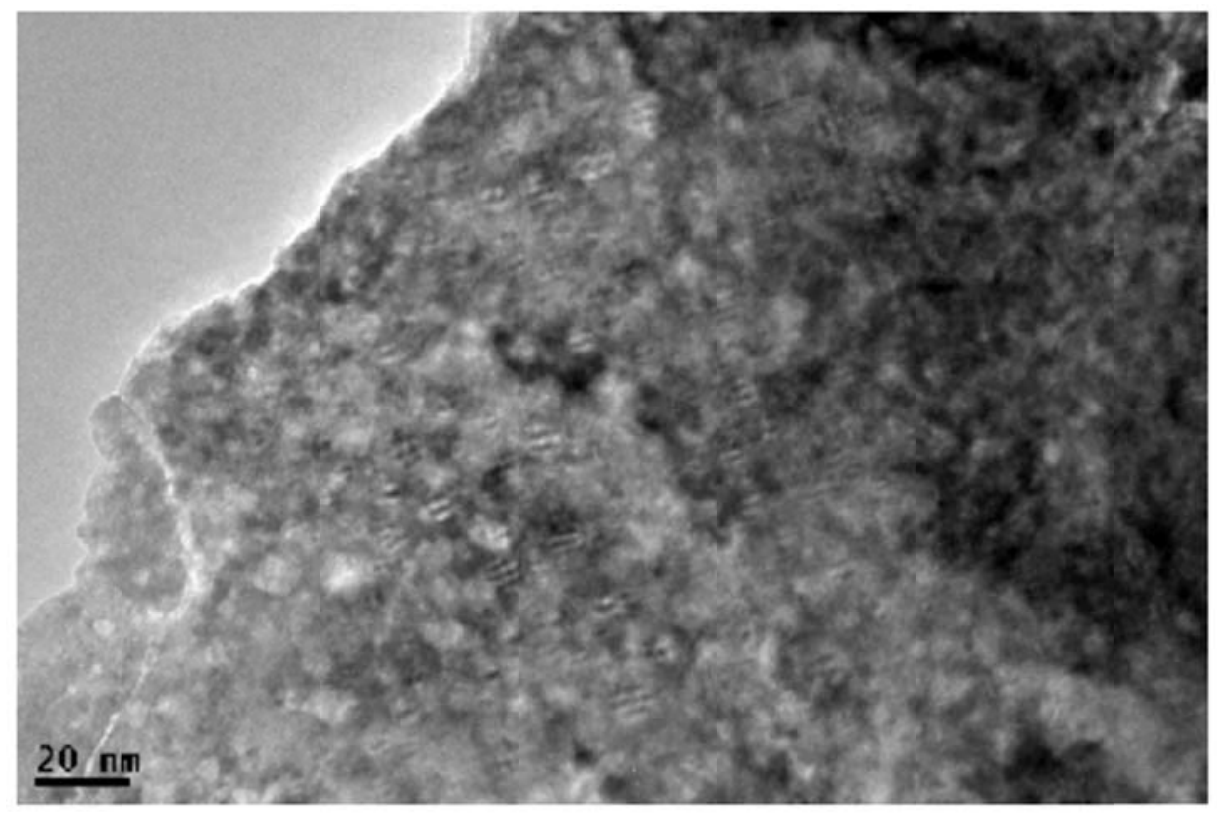

Figure 2.18. Illustration of possible solid state Xe precipitates in 5\% La doped CeO2 with $700 \mathrm{keV}$ Xe ex situ irradiations at $600 \mathrm{C}$ to a dose of $1 \times 10^{17}$ ions $/ \mathrm{cm}^{2}$

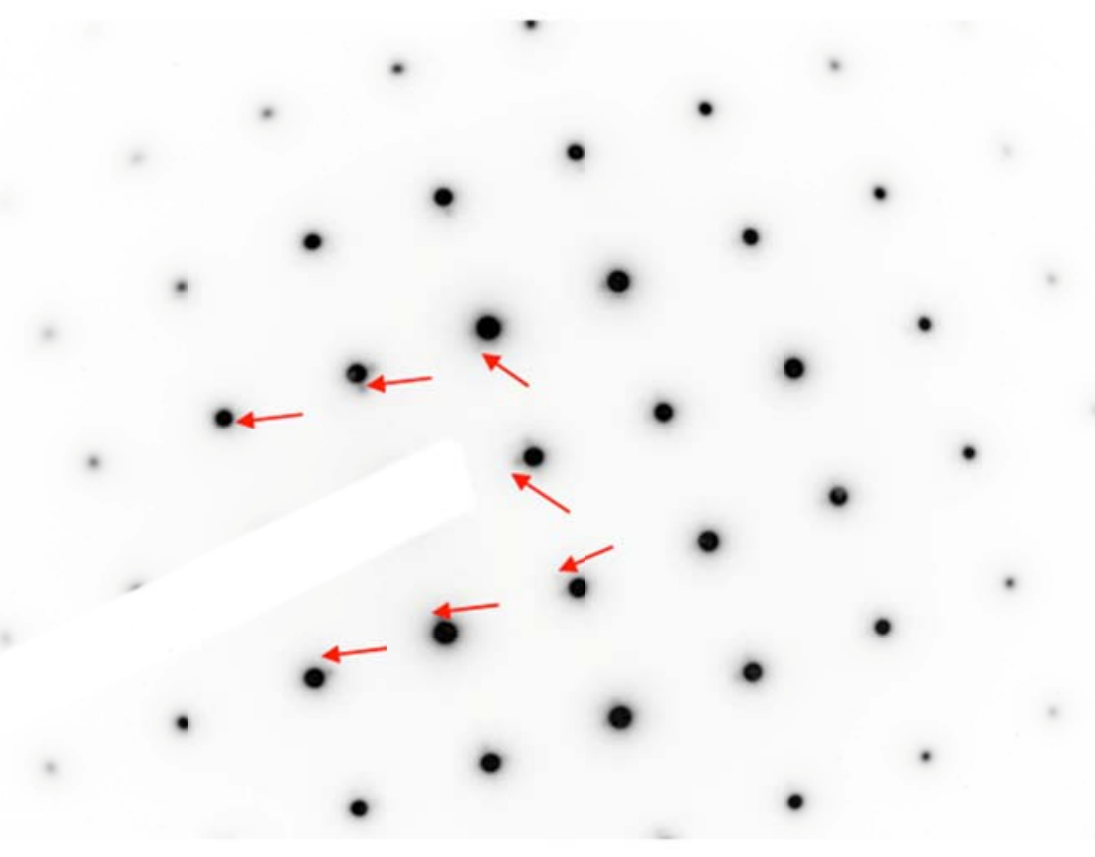


Figure 2.19. SAD diffraction pattern of the possible solid state Xe precipitates as satellite diffraction spots can be seen from this pattern ( the contrast is totally inversed for this image to better show the existence of extra diffraction spots, which are marked by red arrows)

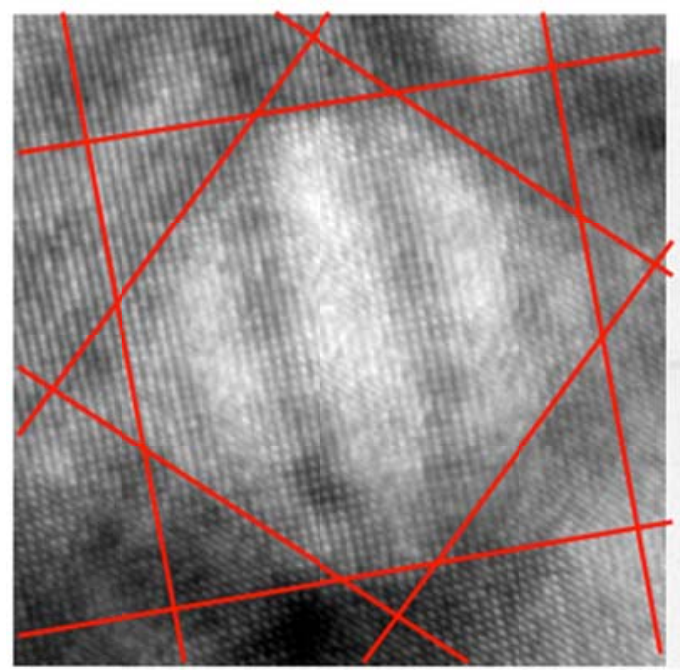

Figure 2.20. (a) HRTEM image of the possible solid state Xe precipitate and (b) the associated FFT showing the facets of the structure

\section{MD Simulation on Stoichiometry Effects on the Evolution of Dislocation Loops during Irradiation}

Atomistic simulation techniques are possible to be used to characterize the structural configurations of these dislocation loops formed in irradiated ceria, which are specifically described in section (c). In this part of the report, MD simulation method is applied to examine the stoichiometry effect due to the lanthanum doping on the evolution of the dislocation loops.

A reliable classical potential is essential to conduct the MD simulation. Gotte et al.'s Buckingham form potential [27] with Coulomb term has been proved accurate in predicting the defect evolution behaviors, especially the diffusion procedure, in cerium oxide system. Still, the potential needs to be expanded for the ceria system with lanthanum dopants. To establish compatible potential parameters for the lanthanum-related interaction, the electron gas method is utilized to calculate the Born-Meyer part of the potential energy profile. Meanwhile, the van der Waals dispersion part of the potential is estimated by means of the Kirkwood method. All the involved potential parameters are listed in Table 2.1. All the cation-cation interaction parameters are ignored due the domination of the Coulomb repulsion. In addition, the ZBL potential is used to modify the Buckingham potential within the very short range.

Table 2.1 Potential Parameters

\begin{tabular}{cccc}
\hline \multirow{2}{*}{ Pair } & $\mathrm{A}$ & $\rho$ & $\mathrm{C}$ \\
\cline { 2 - 4 } & $\mathrm{eV}$ & $\AA$ & $\mathrm{eV}^{6}$ \\
\hline Ce-0 & 755.1311 & 0.429 & 0.0 \\
$0-0$ & 9533.421 & 0.234 & 224.88
\end{tabular}


First of all, the Frenkel pair evolution simulation is used to examine the lanthanum doping effect. In the material with fluorite structure, due to the excellent radiation tolerance, the consequence of a displacement cascade is no more than some randomly distributed Frenkel pairs rather than the interstitial shell-vacancy core structure formed in metallic materials. Therefore, the randomly distributed Frenkel pair is a good initial condition for MD simulation to predict the evolution of radiation induced defects. In addition, the Frenkel pair evolution method make it possible to study on the anion or cation defect separately so that more valuable information about the defect evolution can be revealed.

The ceria system including $16 \times 16 \times 16$ conventional cells are doped with $0 \%, 5 \%, 10 \%, 15 \%$, $20 \%$ and $25 \%$ of lanthanum. After the systems are relaxed for $2 \mathrm{~ns}$, pure oxygen (350), pure cation (175) and mixed Frenkel $(175+350)$ pairs are randomly introduced into these systems respectively. Then those systems are evolved for another hundreds of picoseconds and the final defect structures are carefully examined. The NpT ensemble is used here for zero pressure and 1000K temperature.

For the pure oxygen Frenkel pair cases, the survived Frenkel pair population verses time data is shown in Figure 2.21. The oxygen Frenkel pairs are not stable in ceria. Actually, few oxygen point defects can endure more than 200ps. More importantly, the lifetime of the oxygen Frenkel pair decreases as the doping concentration rises. For the doping ration larger than $10 \%$, no oxygen point defects exist after 2 ps. This can be easily understood since the rise of the doping results in the increase of the intrinsic oxygen vacancy.

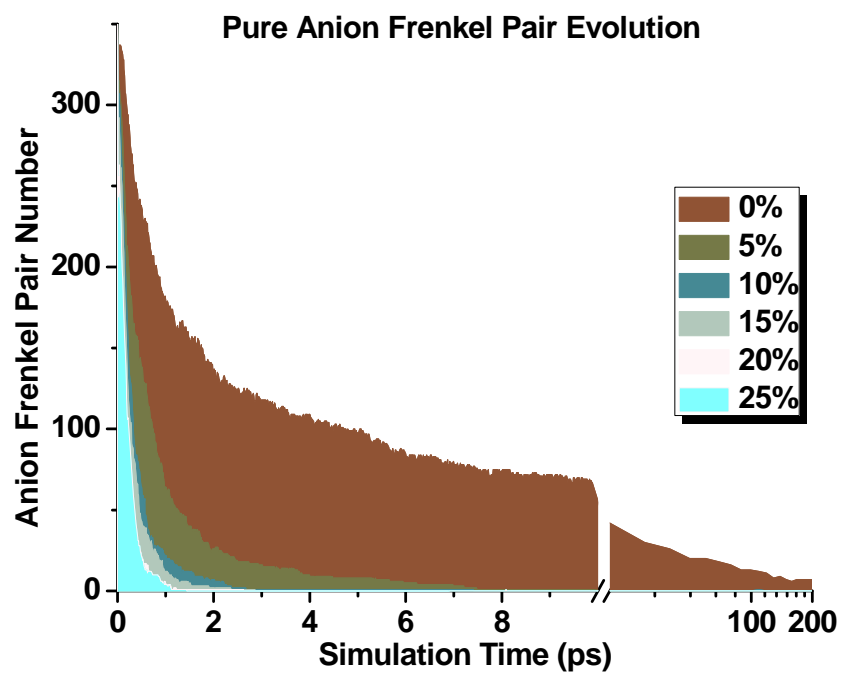

Figure 2.21 Oxygen Frenkel Pair Evolution

For the pure cation Frenkel pair cases, the survival cation Frenkal pair population versus time data is listed in Figure 2.22. The lanthanum ratio of the introduced Frenkel pairs and the local cation type environment do not affect the evolution significantly. In the end, around $80 \%$ of the Frenkel pairs survive the 400ps simulation. In fact, in the $2 \mathrm{~ns}$ simulation, the survival population has only marginal reduction. Thus, the cation point defect is quite stable within the MD timescale 
due to the low mobility. On the other hand, the planar interstitial clusters are formed in the simulation. More importantly, oxygen Frenkel pairs are produced by the system spontaneously to help neutralize the charge bias of the cation interstitial clusters, which indicates that the cation Frenkel pairs dominate the formation of the dislocation loops. However, the stoichiometry effect is not prominent in this stage of the simulation.

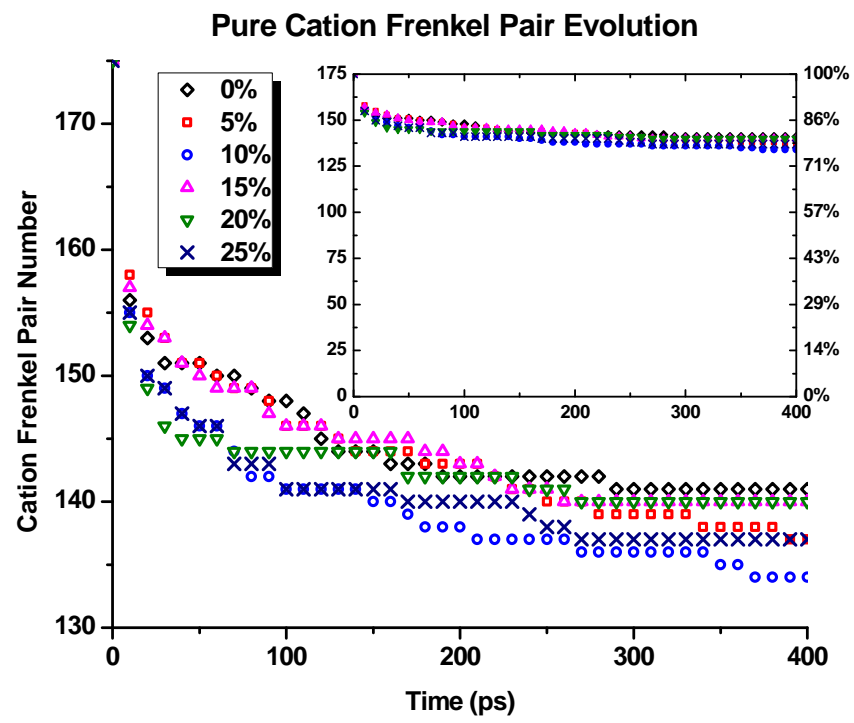

Figure 2.22 Cation Frenkel Pair Evolution

For the mixed Frenkel pair cases, the final defect structures are shown in Figure 2.23 compared with the corresponding pure cation Frenkel pair cases (with identical initial cation Frenkel pair positions). As the lanthanum doping concentration is high, the mixed Frenkel pair cases are similar to the mixed cases. However, when the doping concentration is low, more and larger dislocation loops are observed in the mixed Frenkel pair cases. Also, the final survival numbers of the cation Frenkel pairs are listed in Table 2.2. Both the loop formation and cation Frenkel pair survival situations indicate that the mobility of cation interstitial rises with the existence of the initial oxygen Frenkel pairs as the doping ratio is low, which can also be related to the longer lifetime of oxygen Frenkel pairs and fewer lanthanum cations in the low doping cases.

Table 2.2 Comparison of the Survival Cation Frenk Pairs

\begin{tabular}{|c|c|c|c|c|c|c|}
\hline Doping Concentration & $0 \%$ & $5 \%$ & $10 \%$ & $15 \%$ & $20 \%$ & $25 \%$ \\
\hline Pure Cation & 142 & 137 & 141 & 140 & 144 & 145 \\
\hline Mixed & 125 & 136 & 142 & 137 & 144 & 148 \\
\hline
\end{tabular}

Although the Frenkel pair evolution simulation can not only predict the defect structures formed in irradiated ceria, but also focus on cation and anion point defects separately, it lacks the capability of modeling the effect of the subsequent cascade on the previously produced defects. Thereby, the displacement cascade overlaps simulation is still necessary. In the cascade simulations, the ceria systems including $25 \times 25 \times 25$ conventional cells with $0 \%, 5 \%$ and $25 \%$ doping are relaxed for $2 \mathrm{~ns}$ before the introduction of twenty $10 \mathrm{keV}$ PKA's. The $10 \mathrm{keV}$ kinetic energy is close to the limit of producing sub-cascades. Each PKA is introduced only when the 
energy introduced by the previous one is eliminated by heat transfer. The temperature of the edge layers are controlled at $1000 \mathrm{~K}$ whereas the energy conservation is kept for the central atoms. The defect structures formed are well monitored after each cascade.

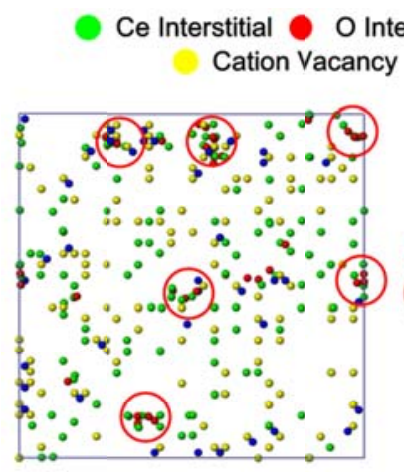

Pure, Cation FP

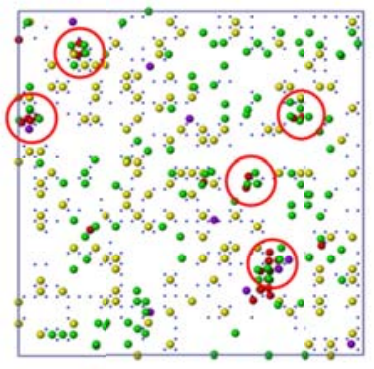

$5 \%$, Cation FP

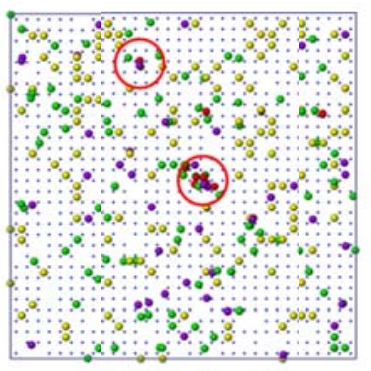

$25 \%$, Cation FP

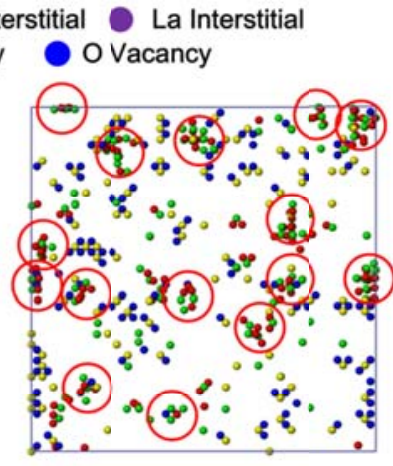

Pure, Mixed FP

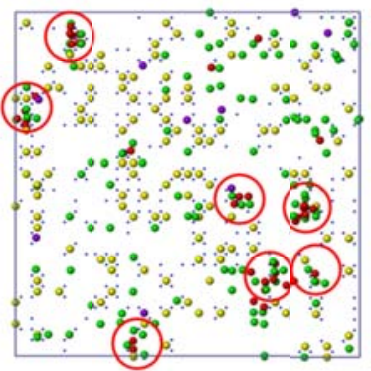

$5 \%$, Mixed FP

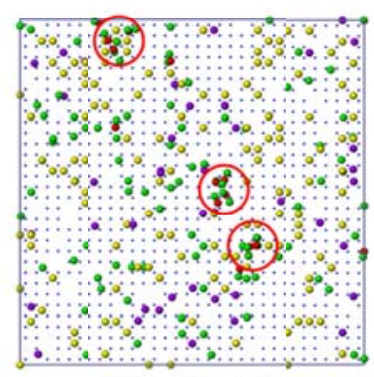

$25 \%$, Mixed FP

Figure 2.23 Final Defect Structures for Cation and Mixed Frenkel Pair Cases

The final defect structural configurations are very similar to the mixed Frenkel pair evolution cases. The loop size distribution situation is illustrated in Figure 2.24. The interstitial type dislocation loops are formed in all the doping cases. However, only as the doping concentration is low, the numerous larger loops can be formed. Moreover, the survival cation Frenkel pair numbers are also listed in Figure 2.25. The survival cation Frenkel pair population rises with the doping concentration, which is also consistent with the results of the Frenkel pair evolution simulations. Another interesting discover is that the lanthanum ratio of the cation interstitials is always much higher than the bulk doping concentration (see Figure 2.26). This phenomenon also happens in the Frenkel pair evolution simulations despite of the poor prominence. The cause of this lanthanum ratio bias is due to the fact that the lanthanum interstitial has an energy preference of $0.5 \mathrm{eV}$ compared with the cerium one. 
With the simulation results from both the Frenkel pair evolution simulation and the cascade overlaps, we can conclude the stoichiometry effect on the evolution of the loops. The nucleation of the loop is dominated by the cation interstitials. Although the isolated cation interstitial is immobile within the MD timescale, the energy barrier of migration is largely lowered when the cation interstitials are closely distributed so that the loop can be formed. Then the oxygen interstitials help neutralize the charge of the loop structure. In the meantime, the existence of the oxygen Frenkel pairs can further raise the mobility of the cation interstitial. When the doping exists, the lifetime of the oxygen Frenkel pairs is shortened, resulting in the lower mobility of the cation interstitial, which can cause bother the suppression of the loop formation and the cation Frenkel pair annihilation. Also, the preference of the lanthanum interstitial also reduces the mobility of cation interstitial as the doping ratio is high. Competition mechanisms due to the lanthanum dopant are then found. However, the simulation methods with longer timescale, such as rate theory and $\mathrm{kMC}$, are needed to examine the long-term behaviors of accumulation of the point defects in high doping concentration cases (25\%).

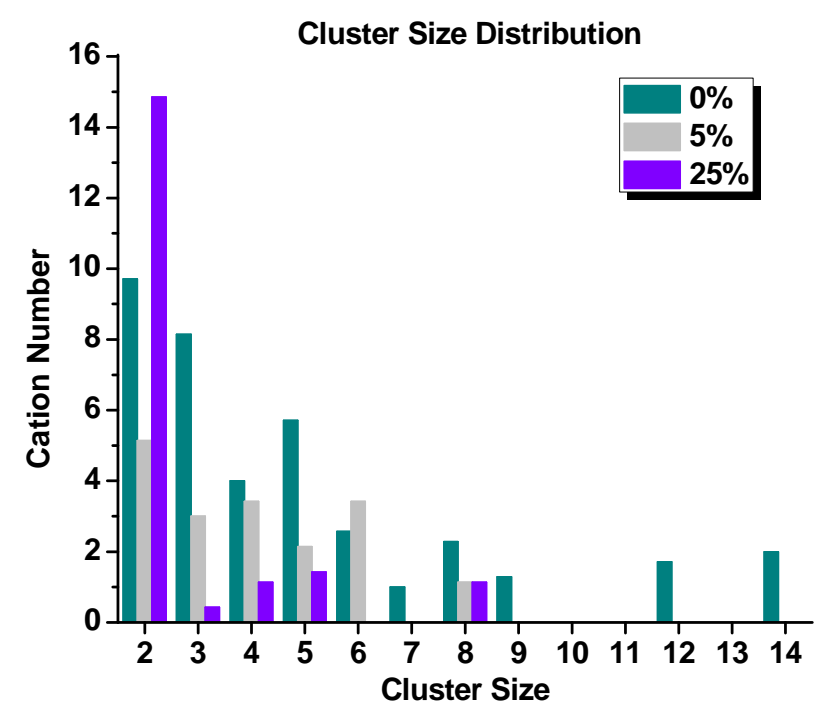

Figure 2.24 Loop Size Distributions in Cascade Overlaps Simulations 
Cation Frenkel Pair Statistics, 20 Cascades

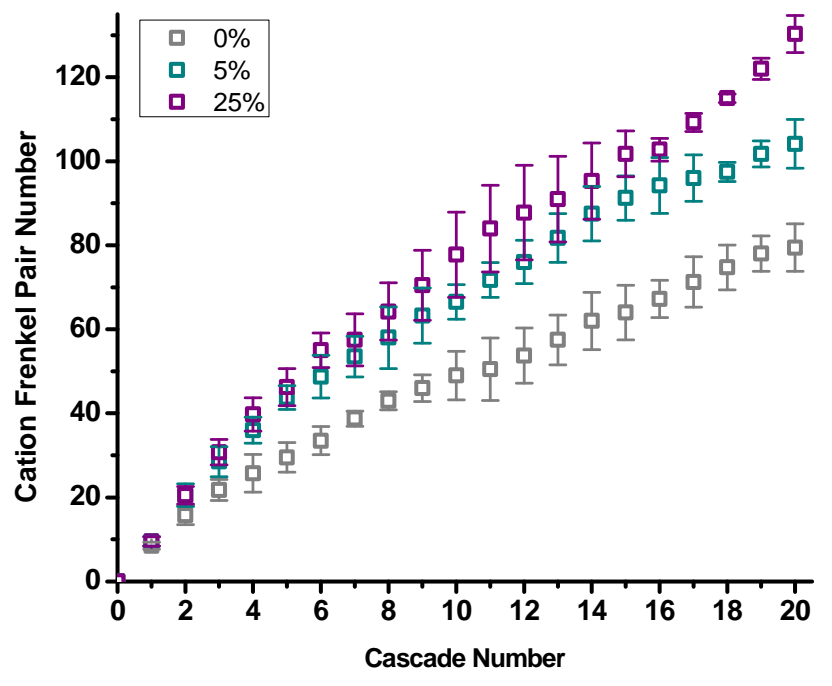

Figure 2.25 Cation Frenkel Pair Populations in Cascade Overlaps Simulations

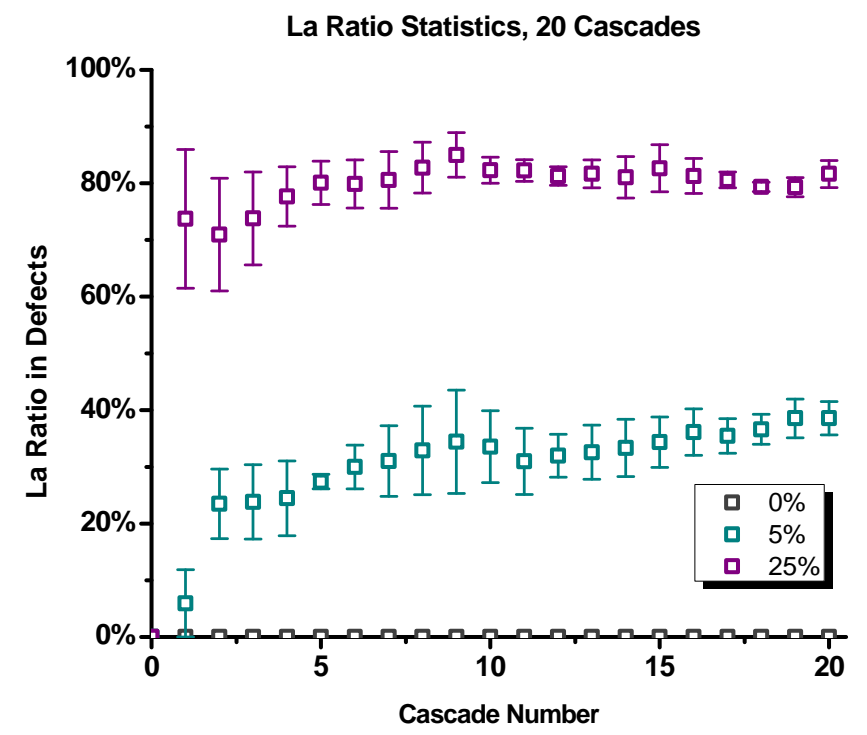

Figure 2.26 Lanthanum Ration of the Cation Interstitial

\section{MS, kMC Modeling of Stoichiometry Effects on Oxygen Diffusivity in $\mathrm{CeO}_{2}$}

The stoichiometry introduced by the lanthanum doping also affects the oxygen diffusivity. Since the lanthanum dopant is a trivalent cation, every two doped lanthanum introduce an oxygen vacancy into the system. Thus, these intrinsic oxygen vacancies make the thermal activation of oxygen Frenkel pairs unnecessary for the diffusion process, raising the oxygen diffusivity a lot compared with that of the pure ceria. However, as the doping concentration is high, the previous experimental results indicate the reduction of the oxygen diffusivity (See Figure 2.27) [28]. To better understand this phenomenon, kMC simulation is conducted based on the parameters calculated by molecular statics. 


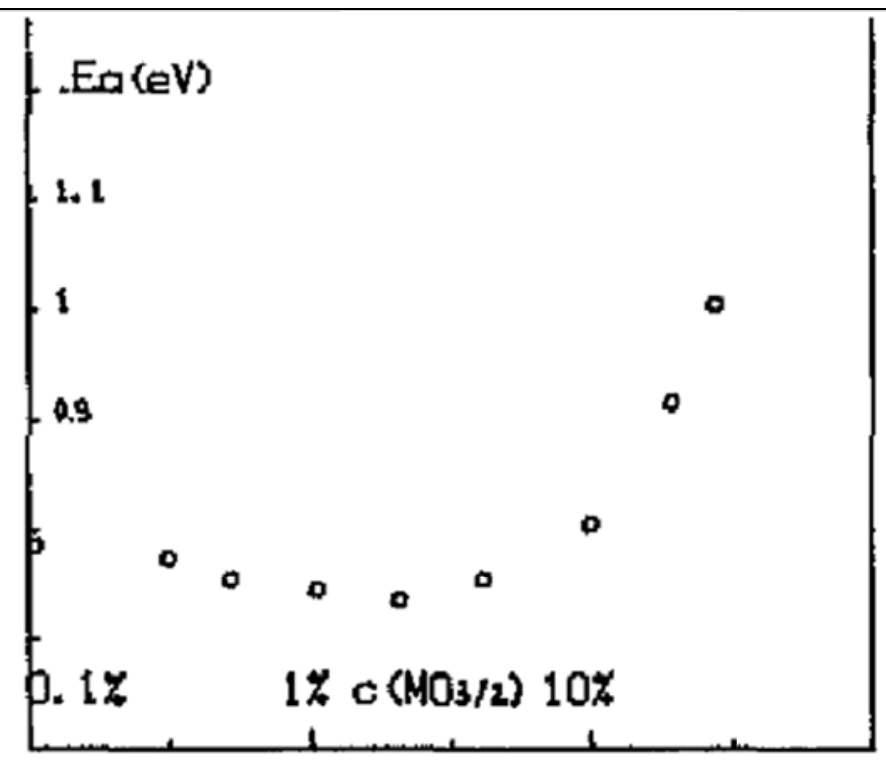

Figure 2.27 Effective Activation Energy of Oxygen Vacancy Migration [28]

The statics calculation code, GULP, is utilized in this study to obtain the migration energies of oxygen vacancies in different position configurations. Multiple classical potentials are used. Gotte et al.'s (27) potential can perfectly duplicate the experimental results. Hence, only the results from this potential are discussed in the following paragraphs.

As shown in Figure 2.28, two anion lattice positions, the start and destination point, are involved in the oxygen vacancy migration process. Since the atom types (cerium or lanthanum) of the cations near the migration positions significantly influence the migration energy, the 6 nearest cation lattice positions (as marked in Figure 2.28) are considered in this study whereas other cations are ignored. Thus, there are 64 different configurations for these six cations with only 30 unique ones due to the symmetry.

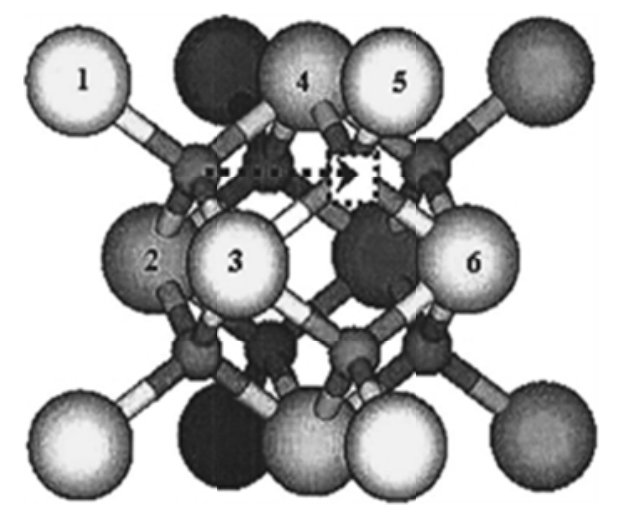

Figure 2.28 Oxygen Vacancy Migration

The migration energies of all those 30 unique configurations are calculated by GULP code with $4 \times 4 \times 4$ conventional cell size and $12-25 \AA ̊$ Mott-Littleton radii. The rational functional optimizer (RFO) technique is used to obtain the first-order saddle point of the potential energy. Some typical values of the migration energies are listed in Table 2.3. The unabridged data can be found in Oaks's thesis.

Table 2.3 Typical Values of the Oxygen Vavancy Migration Energies 
Fundamental Studies of Irradiation-Induced Defect Formation and Fission Product Dynamics in Oxide Fuels

\begin{tabular}{cccc} 
La Position & Em $(\mathrm{eV})$ & La Position & Em(eV) \\
\hline 1 & 0.34531 & 5 & 0.49828 \\
1,2 & 0.06541 & 5,6 & 0.67827 \\
1,3 & 0.98412 & 3,5 & 1.16797 \\
$1,2,3$ & 0.78516 & $3,5,6$ & 0.88295 \\
\hline
\end{tabular}

Then, these migration energy data are used as parameters for the kMC simulation. Dr. C. Deo's kMC code generalized by Oaks is used in this study. A system including $15 \times 15 \times 15$ conventional cell with periodical boundary condition is doped with various amount of lanthanum. The system temperature is set as 800C and 500,000 kMC steps are run for each case. The oxygen vacancy diffusivity can be easily calculated by counting the mean square displacement of oxygen vacancies within the system.

$$
D_{v}=\frac{<x_{v}^{2}>}{6 t}
$$

And the oxygen diffusivity, which is dominated by the vacancy mechanism, can be obtained by:

$$
D_{o}=\left[V_{o}\right] D_{v}
$$

where, $\left[V_{o} \cdot\right]$ is the oxygen vacancy concentration. The final results are illustrated in Figure 4.3. 500,000 steps are sufficient to reach convergence. Also, the peak of the diffusivity happens around 3\% doping concentration, which is consistent with the experimental results.

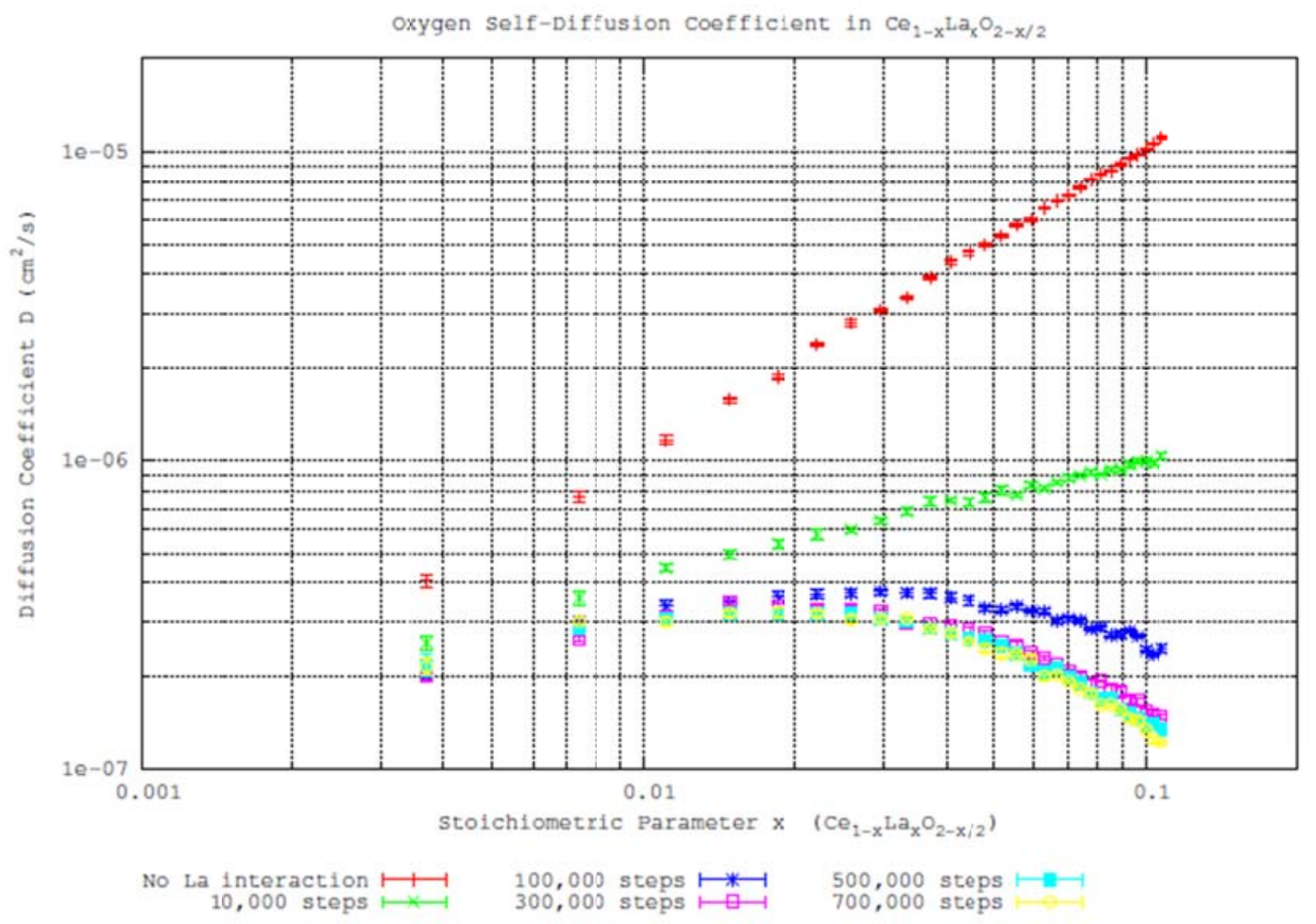

Figure 2.29 Oxygen Diffusivity versus Stoichiometry 


\section{E. Characterization of Dislocation Loops in $\mathrm{CeO}_{2}$}

\section{TEM Characterization}

The habit plane of a dislocation loop is the crystallographic plane containing the dislocation line. To determine the habit planes of dislocation loops, the specimen was tilted with a doubletilt holder in a TEM. At certain orientations, the projections of some dislocation loops will become edge-on. Figure 2.30 shows a bright field image of an irradiated $\mathrm{CeO}_{2}$ specimen, and its diffraction pattern. The specimen was carefully tilted so that the direction of electron beam is close to the [011] zone axis, and only $\{200\}$ reflections are strongly excited. The line segments in the image are perpendicular to the (111) and (1111) diffraction vectors, so the edge-on

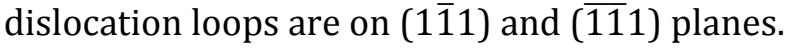

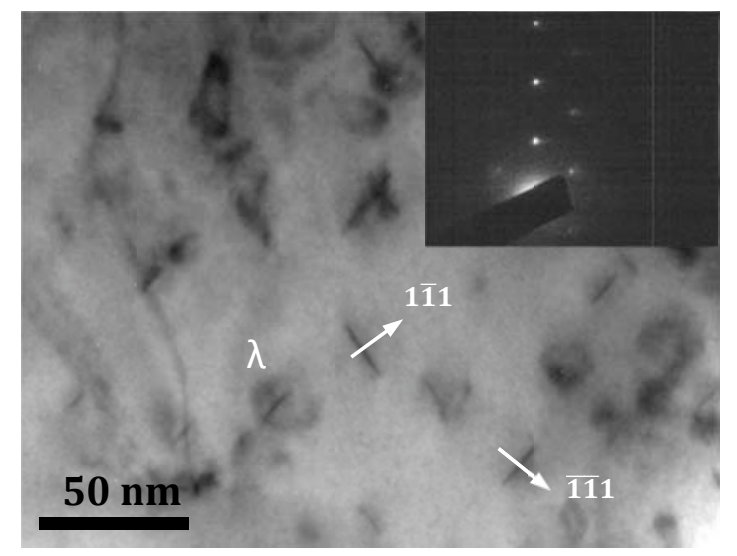

Figure 2.30. Bright field TEM image of irradiated $\mathrm{CeO}_{2}$ when electron beam is close to [011]. The inset is the diffraction pattern showing that (200) reflection is excited for imaging.

Below the $\lambda$ symbol in figure 1 are two dislocation loops. One is an edge-on loop appearing as a line segment, and the other is an inclined loop (indicated as loop $\lambda$ afterward) appearing as grey ellipse.

The fluorite crystal structure is derived from the fcc structure, and thus has an ABCABC stacking sequence on $\{111\}$ planes. In this context, a stacking fault is defined as a discontinuity or fault in the stacking sequence. A dislocation loop surrounded by partial dislocations would result in such a stacking fault, and would appear in TEM images as interference fringes inside the boundary of the loop. Figure 2.31 shows a pair of dark field and bright field TEM images observed with diffraction vector $\mathrm{g}=(111)$ near the $[\mathbf{1 1} \overline{2}]$ zone axis. The round features with fringes perpendicular to the [111] direction are stacking fault dislocation loops on the (11) plane. Therefore, those $\{111\}$ dislocation loops have Burgers vectors that lead to their stacking fault nature. In the fcc lattice, $1 / 6<112>$ and $1 / 3<111>$ are the most commonly observed Burgers vectors of a partial dislocation that can result in a stacking fault. The Burgers vector of dislocation loops in this study will be discussed in the next section. 


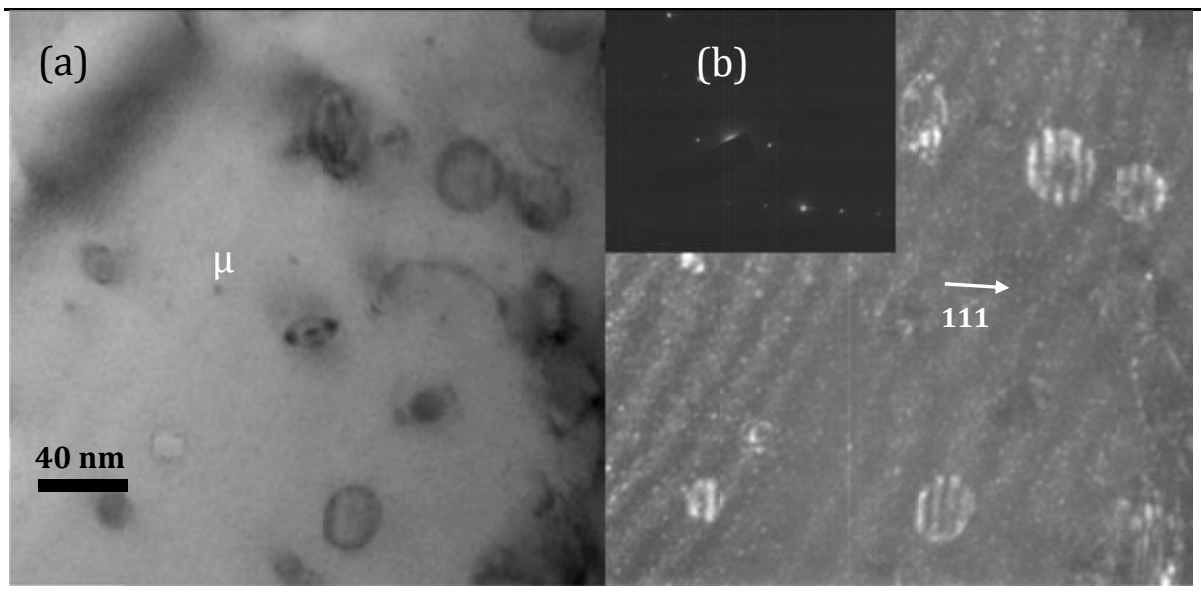

Figure 2.31. The (a) bright field and (b) dark field TEM images of $\mathrm{CeO}_{2}$ irradiated with $1 \mathrm{MeV} \mathrm{Kr}$ at $800{ }^{\circ} \mathrm{C}$ to a dose of $5 \times 10^{14}$ ions $/ \mathrm{cm}^{2}$. The inset on the top-left corner of (b) is the diffraction pattern showing strong (111) reflections at [112] zone.

The Burgers vectors of the stacking fault dislocation loops on $\{111\}$ planes are determined by $b \cdot g$ analysis. Figures 2.32 and 2.33 show a series of bright and dark field TEM images on the same area with various diffracting conditions. Dislocation loops herein were grouped by character into four designations (A through D). In figure 2.32(a) $(g=(020))$, all dislocation loops are visible. In figures 2.32 (b) and 2.32 (c) (g=(220)), types B and D are out of contrast $(b \cdot g=$ $0)$, and thus become invisible. Similarly, in figures $5(\mathrm{~d})$ and 2.32 (e) ( $g=(\overline{2} 20))$, types A and C are out of contrast, and thus become invisible. Without a loss of generality, we consider only $<111>$, $<220>$, $<200>$ and $<112>$ directions for candidate Burgers vectors. Applying $b \cdot g$ analysis narrows down the possible Burgers vectors to [111], [111] or [220] directions for types $A$ and $C$, and to [1 $\overline{1} 1],[\overline{1} 11]$ or $[2 \overline{2} 0]$ directions for types B and D.

Reflections $(4 \overline{22})$ and $(42 \overline{2})$ were used to finalize the $b \cdot g$ analysis. In figures 2.33(a) and 2.33 (b) ( $g=(4 \overline{22})$ ), type A loops are out of contrast and become invisible, while all other types are still visible. Due to the very weak diffracting condition used, type C loops in figure 2.33 (a) looks almost as out of contrast as type A loops. A stronger diffracting condition image was taken in figure 2.33 (b) to show that type $C$ loops are in fact in contrast with $(4 \overline{22})$ reflection whereas loops type A remain invisible. Thus, type A loops satisfy the invisibility criterion with both ( $2 \overline{2} 0)$ and (4 $\overline{22}$ ) reflections, so type A loops must have Burgers vectors in the [111] direction. Similarly, in figure 6(c) $(\mathrm{g}=(42 \overline{2})$ ), type B loops are out of contrast and become invisible. Thus, type B loops satisfy the invisibility criterion with both (220) and $(42 \overline{2})$ reflections, so type B loops must have Burger's vectors in the [1 111$]$ direction. Although specific examinations for types $C$ and $D$ were not performed, it is reasonable to suggest that their Burgers vectors are also in $<111>$ directions perpendicular to their habit planes. 
Fundamental Studies of Irradiation-Induced Defect Formation and Fission Product Dynamics in Oxide Fuels
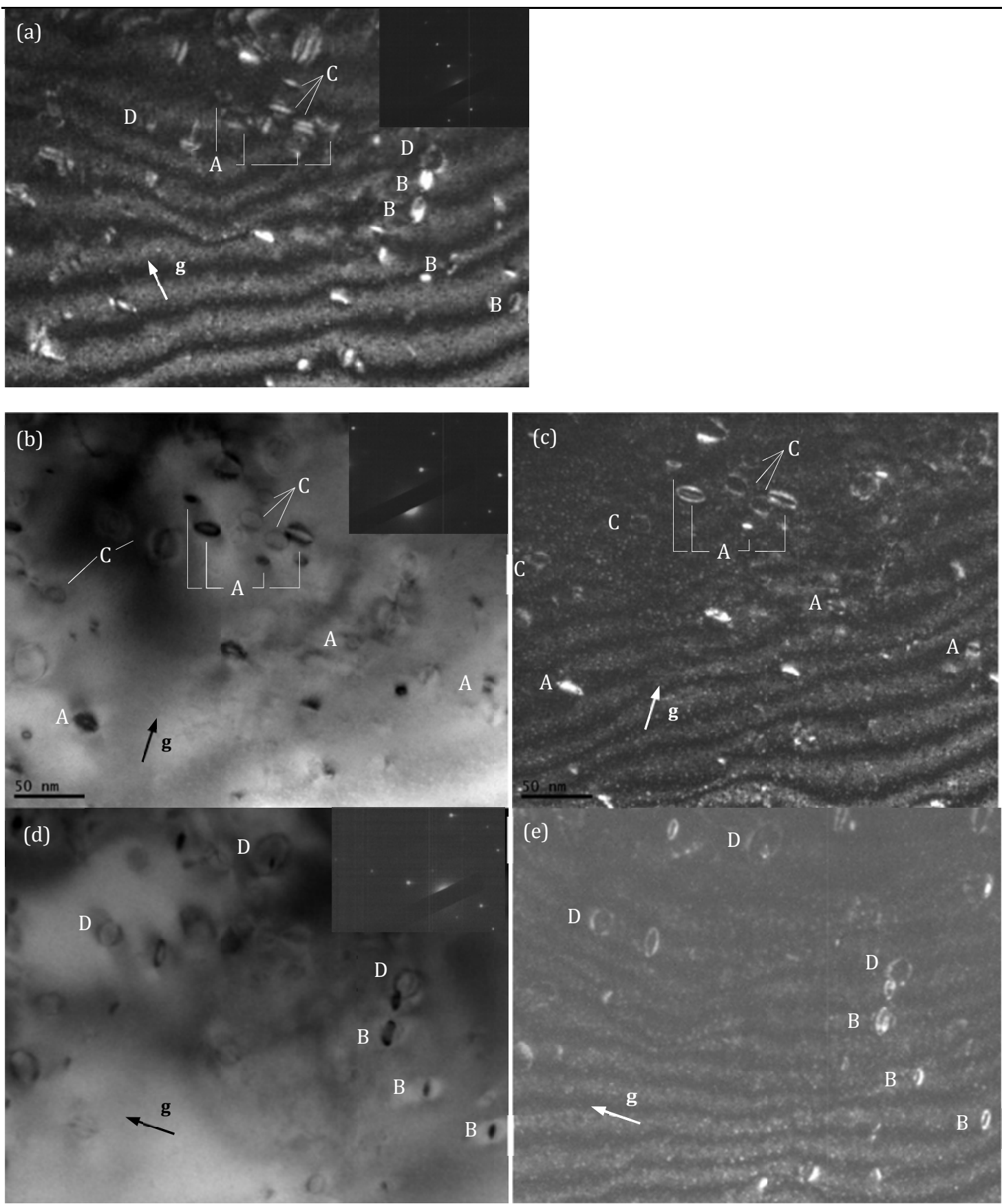

Figure 2.32. TEM images of irradiated $\mathrm{CeO}_{2}$ when electron beam is close to [001] zone: (a) Dark field image with $g=(020)$. (b) Bright field and (c) dark field TEM images with $g=(220)$. (d) Bright field and (e) dark field TEM images with $g=(\overline{2} 20)$. Characters A through D are used to indicate dislocation loops on different groups of $\{111\}$ planes. The insets are the corresponding diffraction patterns. 


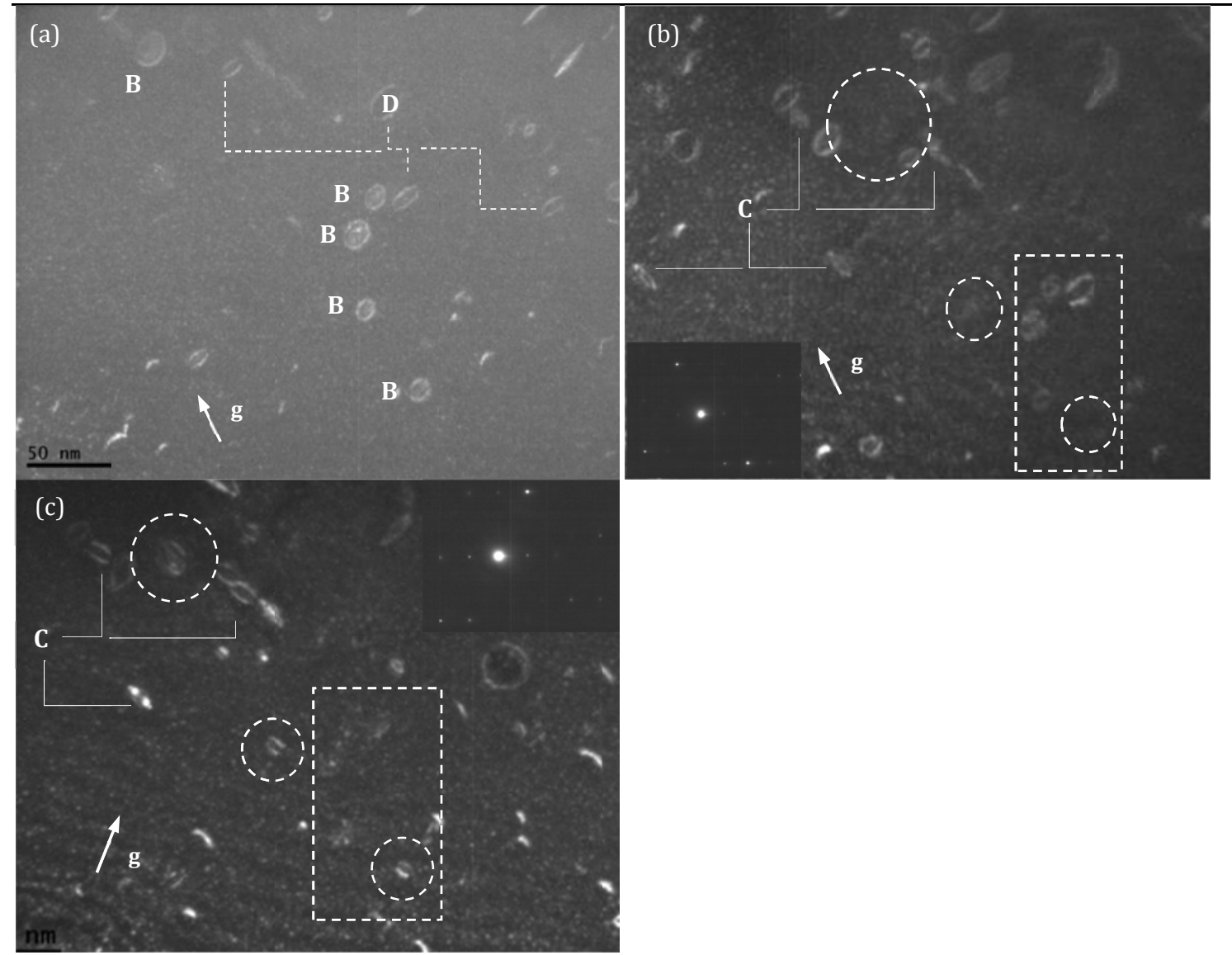

Figure 2.33. Dark field TEM images of irradiated $\mathrm{CeO}_{2}$ when electron beam is close to [102] zone: (a) $g=(4 \overline{2} 2)$ with weaker diffraction condition. (b) $g=(4 \overline{2} 2)$ with stronger diffraction condition. (c) $g=(42 \overline{2})$. Characters $B, C$ and D are to indicate the same loops as in figure 2.32 . The dashed circle is to indicate the locations of loops $\mathrm{A}$ and the dashed square is to indicate the locations of loops B. The insets are the corresponding diffraction patterns.

The nature of the loops was determined from their inside-outside contrast behavior. Careful observation between the Kikuchi band movements and the sense of the foil tilting was performed for establishing their intercorrelation. The sense of inclination of both sets of the loops were then determined by tilting the foil to [101] pole along 020 Kikuchi band. As a result, the habit planes for sets $A$ and $B$ are $(\overline{11} 1)$ and (111), respectively. The projection of upwarddrawn plane normal for loops $\mathrm{A}, \mathrm{n}_{/ / \mathrm{A}}$, are indicated as dashed arrow in figure 2.34. Based on the above knowledge (the sense of inclination and the inside-outside contrast behavior), dislocation loops A and B in figure 2.34 were consistently characterized as having an interstitial nature. 

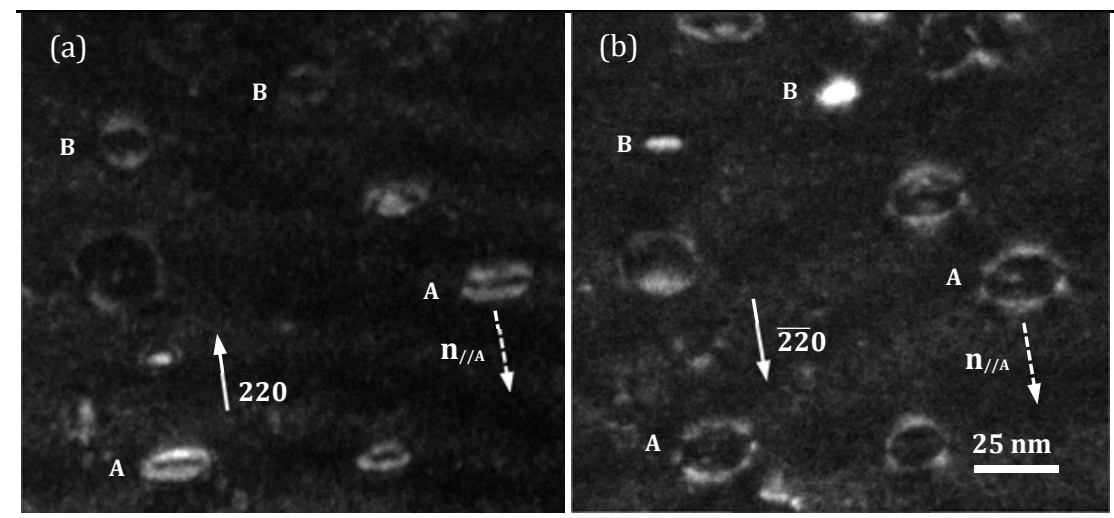

Figure 2.34. Determination of the nature of the loops with inside-outside contrast behavior. The

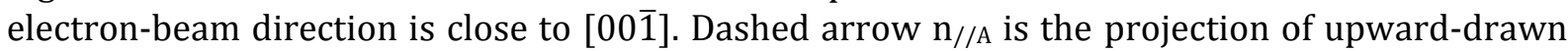
plane normal (upward means out of paper) of loops A. The contrast behavior is consistent with the nature for interstitial dislocation loops.

Although several aspects of dislocation loop properties have been examined and characterized, the magnitude of the Burgers vectors is still underdetermined. In literatures, two types of Burgers vectors for irradiated $\mathrm{CeO}_{2}$ have been proposed: $1 / 9<111>$ and $1 / 3<111>$. The former is composed of a single layer of oxygen interstitials and it is claimed to be due to the irradiating environment that only selectively displaces oxygen lattice ions [29-31]. The latter consists of a stoichiometric stack, consisting of a middle layer of cerium interstitials and two side layers of oxygen interstitials. [32-34]

The dislocation loops observed in this study should be $1 / 3<111>$ type for several reasons. First, under irradiation of $1 \mathrm{MeV} \mathrm{Kr}$ ions, both oxygen and cerium atoms were being displaced. Thus, the defect source configuration in this study is different from studies where only oxygen Frenkel pairs were generated. Beside, the existence of the cerium interstitials make the formation of $1 / 3<111>\{111\}$ loops more energetically favorable over $1 / 9<111>\{111\}$ loops, since the latter is not charge balanced. In addition, $1 / 9<111>\{111\}$ loops have small stress fields due to their small magnitude Burgers vectors, so we suggest that these loops are unlikely to show a stacking fault contrast such as what was seen in figure 2.31 .

In conclusion, several aspects of the dislocation loops induced by $1 \mathrm{MeV} \mathrm{Kr}$ in $\mathrm{CeO}_{2}$ to a dose of $5 \times 10^{14}$ ions $/ \mathrm{cm}^{2}$ at elevated temperatures have been characterized. Loops were determined to be interstitial type lying on $\{111\}$ planes and have Burgers vectors in $<111>$ directions perpendicular to their habit planes. Loops were also found to have a stacking fault nature. In addition, Burgers vectors are suggested to have a magnitude of $1 / 3<111>$.

\section{MD Simulation of the Atomistic Structure of the Dislocation Loops}

As mentioned before, the structure of the dislocation loops observed in irradiated ceria has been determined to be the $\mathbf{b}=1 / 3<111>$ interstitial type Frank partial dislocation loops. Actually, in both the Frenkel pair evolution simulations and the displacement cascade overlaps simulations with any doping concentration, the only stable structure of the larger interstitial cluster is the Frank loop, the typical structure of which is illustrated in Figure 2.35. A better graphic description of the loop structure can be found in Figure 2.36.

As we all know, the primary slip system of the fluorite structure is $\{001\}<110>$, namely, the dislocation should have $\mathbf{b}=1 / 2<110>$. Also, the Frank loops are observed to evolve into 
dislocation lines and then dislocation networks which definitely have $\mathbf{b}=1 / 2<110>$. Therefore, the preference of the nucleation of the interstitial clusters is worth studying. Also, the transition mechanism from the $\mathbf{b}=1 / 3<111>$ to the primary slip system is desired.

So, two most plausible configurations of the interstitial type dislocation loop are considered, the $\mathbf{b}=1 / 3<111>$ Frank loop and $\mathbf{b}=1 / 2<110>$ perfect loop (see Figure 2.36). The defect energy profiles of both types of circular loops including up to $129 \mathrm{CeO}_{2}$ interstitial combinations are calculated by both statics and dynamics simulations. The results are averaged by the $\mathrm{CeO}_{2}$ molecule number in the interstitial type loop for better comparison. The results are shown in Figure 2.37. As the loop size is small, the Frank loop has a prominent preference of nucleation. As the loop consists of fewer than $7 \mathrm{CeO}_{2}$ molecules, the perfect loop is even unstable. On the other hand, as the loop size rises, the stacking fault energy becomes dominant for the Frank loop. As a result, large Frank loops have higher defect energies than the perfect loop. This result can explain the fact that only $\mathbf{b}=1 / 3<111>$ Frank loops are observed in the previous simulations. Also, the energy difference between the Frank loop and perfect loop with large size is sufficient to drive the transition. The transition from the $\mathbf{b}=1 / 3<111>$ loop to $\mathbf{b}=1 / 2<110>$ loop is well understood in FCC metallic material. The dislocation reaction can be described as $1 / 6[\overline{1} 2 \overline{1}]+$ $1 / 6[2 \overline{11}]+1 / 3[111] \rightarrow 1 / 2[110]$. Here, two Shockley partial dislocations are spontaneously nucleated to assist the Frank loop to turn into a mixed perfect loop. Therefore, as the loop is small, since the Frank partial loop is sessile, the loop growth is majorly due to the absorption of new interstitials nearby. When the loop is large enough to transit to a perfect loop, since the perfect loop is able to glide, the loop coalescence is plausible so that the loop growth is enhanced. With the rise of dose, the large loops touch the surfaces and then become perfect dislocations in primary slip system.

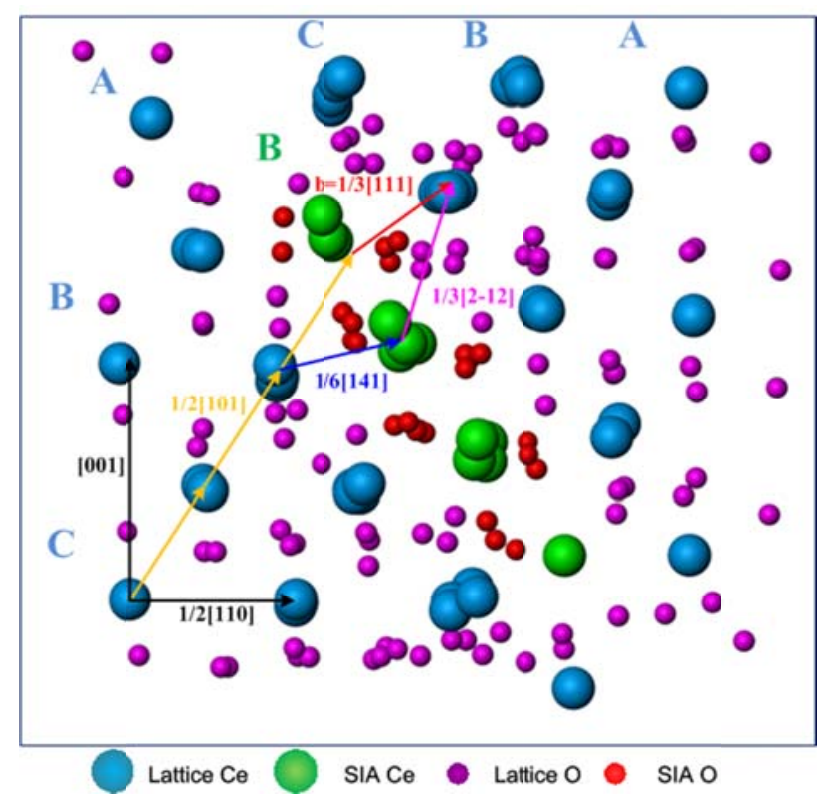

Figure 2.35 Frank Loop Formed in Cascade Overlaps in Pure Ceria 


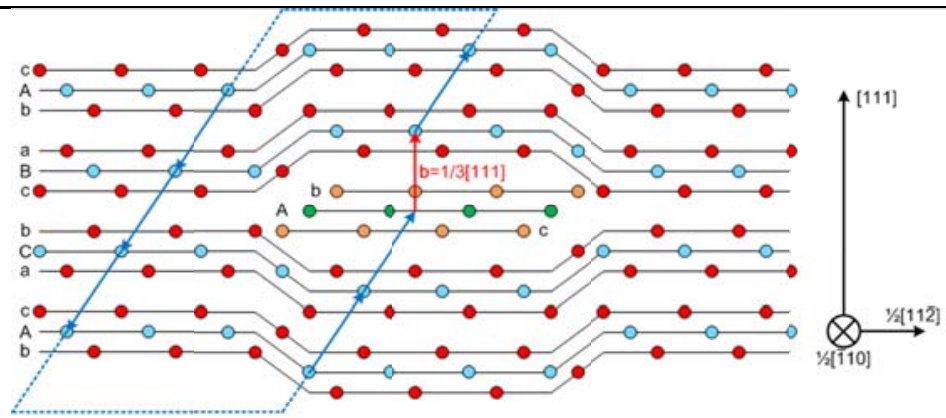

a) $\mathbf{b}=1 / 3<111>$ interstitial type Frank loop

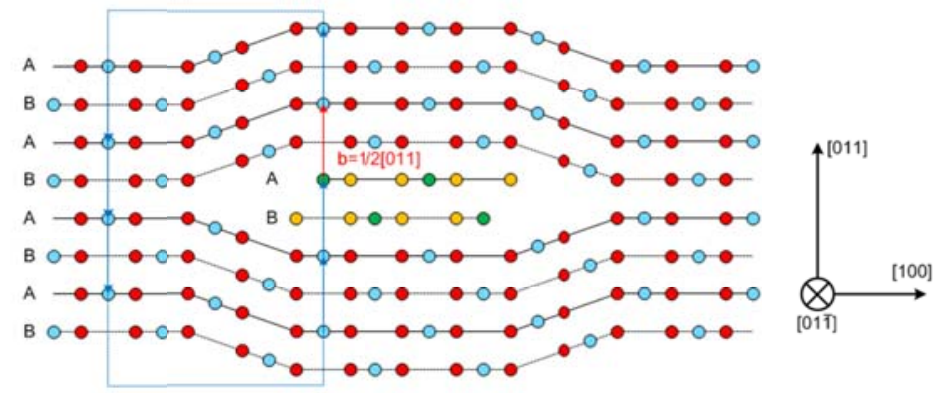

b) $\mathbf{b}=1 / 2<011>$ interstitial type pure edge loop

- Lattice Ce - Lattice O Loop Ce - Loop O

Figure 2.36 Loop Structure Diagram

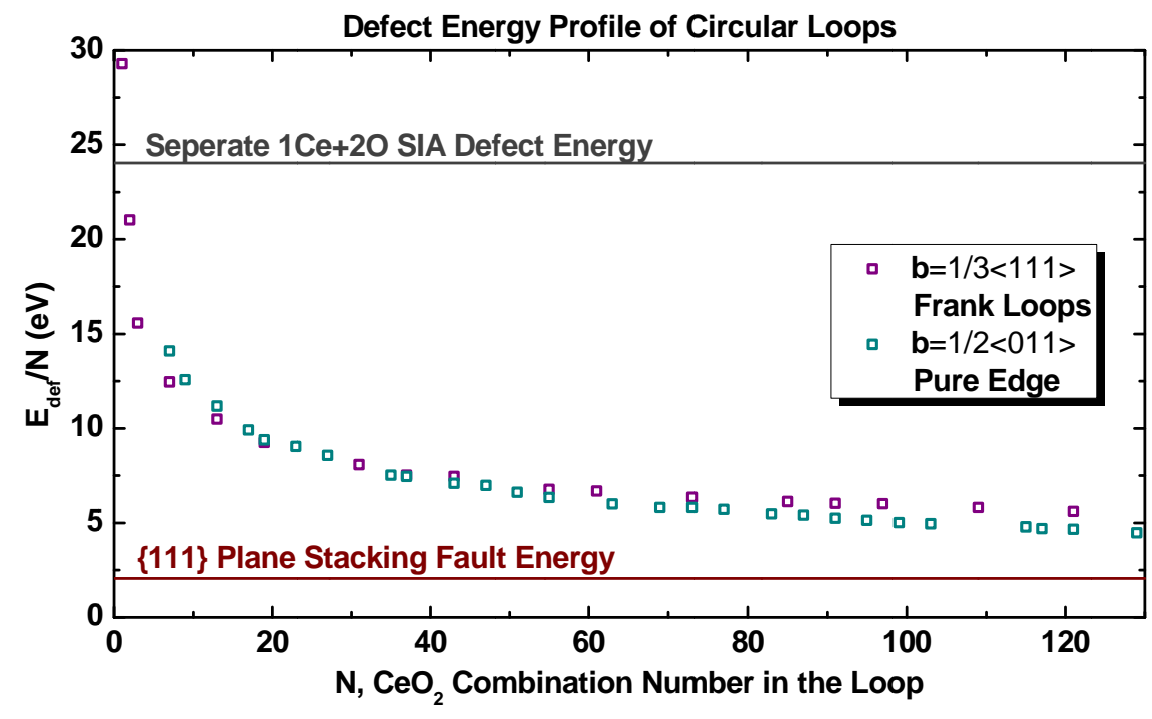

Figure 2.37 Defect Energy Profiles of Two Types of Loops 
IV. REFERENCES

[1] J.P. Raison, T. Mizuno, F. Delage, J. Carmack, Chan Bock Lee, "Sodium Fast Reactor Fuel Evaluation: Oxide Fuels", Transactions of the American Nuclear Society, vol. 98, pp. 1052-1055, 2008

[2] V.V. Rodinella and T. Wiss, "The High Burn-up Structure in Nuclear Fuel", Materials today, vol. 13, pp. 24-32, 2010

[3] Hj. Matzke, "On the Rim Effect in High Burnup $\mathrm{UO}_{2}$ LWR fuels", Journal of Nuclear Materials, vol. 189, pp. 141-148, 1992

[4] J.A. Hinks, "A Review of Transmission Electron Microscopes with In Situ Ion Irradiation", Nuclear Instruments and Methods in Physics Research B, vol. 267, pp. 3652-3662, 2009

[5] J.F. Ziegler, J.P. Biersack, et al. "The Stopping and Range of Ions of Solid", Pergamon Press, New York, 1985

[6] K. Yasunaga, K. Yasuda, S. Matsumura, T. Sonoda, "Nucleating and Growth of Defect Clusters in $\mathrm{CeO}_{2}$ Irradiated with Electrons", Nuclear Instruments and Methods in Physics Research B, vol. 250, pp. 114-118, 2006

[7] D.R. Olander, "Fundamental Aspects of Nuclear Reactor Fuel Elements", Energy Research and Development Administration, 1976

[8] K. Yasunaga, K. Yasuda, S. Matsumura, T. Sonoda, "Nucleating and Growth of Defect Clusters in $\mathrm{CeO}_{2}$ Irradiated with Electrons", Nuclear Instruments and Methods in Physics Research B, vol. 250, pp. 114-118, 2006

[9] C. Sabathier, L. Vincent, P. Garcia, F. Garrido, G. Carlot, L. Thome, P. Martin, C. Valot, "In Situ TEM Study of Temperature-induced Fission Product Precipitation in $\mathrm{UO}_{2}$ ", Nuclear Instruments and Methods in Physics Research B, vol. 266, pp. 3027-3032, 2008

[10] F. Garrido, L. Nowicki, G. Sattonnay, T. Sauvage, L. Thome, "Lattice Location of Helium in Uranium Dioxide Single Crystals", Nuclear Instruments and Methods in Physics Research B, vol. 219-220, pp. 196-199, 2004

[11] K. Une, K. Nogita, S. Kashibe, M. Imamura, "Microstructural Change and Its Influence on Fission Gas Release in High Burnup $\mathrm{UO}_{2}$ Fuel", Journal of Nuclear Materials, vol. 188, pp. 65-72, 1992

[12] N. Nakae, Y. Iwata, T. Kirihara, "Thermal Recovery of Defects in Neutron Irradiated $\mathrm{UO}_{2}$ ", Journal of Nuclear Materials, vol. 80, pp. 314-322, 1979

[13] M. Kiritani, N. Yoshida, H. Tanaka, Y. Maehara, “”, Journal of Physics Society Japan, vol. 38, pp. 1677, 1975 
[14] J.H. Evans, A. van Veen, K.T. Westerduin, "A TEM and TDS Study of Gas Release from Bubbles in Krypton-implanted Uranium Dioxide", Journal of Nuclear Materials, vol. 195, pp. 250-259, 1992

[15] J. Rest, "An Analytical Study of Gas-bubble Nucleation Mechanisms in Uranium-alloy Nuclear Fuel at High Temperature", Journal of Nuclear Materials, vol. 402, pp. 179185,2010

[16] S. Kashibe, K. Une, K. Nogita, "Formation and Growth of Intragranular Fission Gas

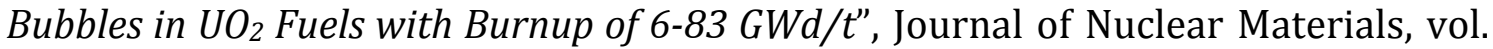
206, pp. 22-34, 1993

[17] A. Iwase, H. Ohno, N. Ishikawa, Y. Baba, N. Hirao, T. Sonoda, M. Kinoshita, "Studay on the Behavior of Oxygen Atoms in Swift Heavy Ion Irradiated $\mathrm{CeO}_{2}$ by Means of Synchrotron Radiation X-ray Photoelectron Spectroscopy", Nuclear Instruments and Methods in Physics Research B, vol. 267, pp. 969-972, 2009

[18] Hj. Matzke, A. Turos, G. Linker, "Polygonization of Single Crystals of the Fluorite-type Oxide UO2 due to Dose Ion Implantation", Nuclear Instruments and Methods in Physics Research B, vol. 91, pp. 294-300, 1994

[19] K. Nogita, K. Une, "High Resolution TEM Observation and Density Estimation of Xe Bubbles in High Burnup $\mathrm{UO}_{2}$ Fuels", Nuclear Instruments and Methods in Physics Research B, vol. 141, pp. 481-486, 1998

[20] A. Trovarelli, "Structural Properties and Nonstoichiometric Behavior of $\mathrm{CeO}_{2}$ ", Eds. A. Trovarelli, Imperial College Press, 2002

[21] D. Yun, Ph. D Dissertation, University of Illinois at Urbana-Champaign, United States, 2010

[22] R. Pornprasertsuk, P. Ramanarayanan, C.B. Musgrave, F.B. Prinz, "Predicting Ionic Conductivity of Solid Oxide Fuel Cell Electrolyte from First Principles", Journal of Applied Physics, vol. 98, pp. 103513-103521, 2005

[23] J. Faber, C. Geoffroy, A. Roux, A. Sylvestre, P. Abelard, A systematic investigation of the dc electrical conductivity of rare-earth doped ceria, Journal of Applied Physics A, Vol. 49, pp.225-232, 1989

[24] Younsuk Yun, Hanchul Kim, Heemoon Kim, Kwangheon Park, Atomic diffusion mechanism of Xe in UO2, Journal of Nuclear Materials, Vol. 378, Issue 1. Pp. 40-44, 2008

[25] K. Mitsuishi, M. Song, K. Furuya, R. C. Birtcher, C. W. Allen, S. E. Donnelly, In situ observation of atomic processes in Xe nanocrystals embedded in Al, Mat. Res. Soc. Symp. Proc., Vol. 504, pp. 417-422, 1998

[26] R. C. Birtcher, S. E. Donnelly, M. Song, K. Furuya, K. Mistuishi, C. W. Allen, Behavior of crystalline Xe nanoprecipitates during coalescence, Physical Review Letters, Vol. 83, No. 8, 1617-1620, 1999 
[27] A.Gotte, D.Spångberg, K.Hermansson, M.Baudin ,Solid State Ionics 178 (25-26) (2007) 1421-1427

[28] J. Faber, C. Geoffroy, A. Roux, A. Sylvestre, and P. Abelard, "A systematic investigation of the dc electrical conductivity of rare-earth doped ceria," Applied Physics aMaterials Science \& Processing, vol. 49, no. 3, pp. 225-232, 1989.

[29] K. Yasunaga, K. Yasuda, S. Matsumura, T. Sonoda, Nucl. Instrum. Methods B 266, (2008) 2877.

[30] K. Shiiyama, T. Yamamoto, T. Takahashi, A. Guglielmetti, A. Chartier, K. Yasuda, S. Matsumura, K. Yasunaga, C. Meis, Nucl. Instrum. Methods B, 268 (2010) 2980.

[31] K. Yasuda, C. Kinoshita, S. Matsumura, A.I. Ryazanov, J. Nucl. Mater., 319 (2003) 74.

[32] D.S. Aidhy, D. Wolf, A. El-Azab, Scripta Mater., 65( 2011) 867.

[33] B. Baufeld, D. Baither, U. Messerschmidt, M. Bartsch, I. Merkel, J. Am. Ceram. Soc., 76 (1993) 3163.

[34] G. Martin, P. Garcia, C. Sabathier, L. Van Brutzel, B. Dorado, F. Garrido, S. Maillard, Phys. Lett. A, 374 (2010) 3038. 
V. PUBLICATIONS AND PRESENATIONS

\section{Journal Papers}

B. Ye, D. Yun, A. J. Oaks, W. Chen, M. A. Kirk, J. Rest, A. M. Yacout, J. F. Stubbins, The Effects of Xenon Implantation in Ceria with and without Lanthanum, presented at IBMM and accepted for publication in Nuclear Methods B.

Bei Ye, Aaron Oaks, Mark Kirk, Di Yun, Wei-Ying Chen, Benjamin Holtzman, Mohamed ElBakhshwan, Brent Heuser and James F. Stubbins, Irradiation Effects in $\mathrm{UO}_{2}$ and $\mathrm{CeO}_{2}$, presented at ANS NFSM and accepted for publication in J Nucl Mater

Di Yun, Bei Ye, Aaron J. Oaks, Wei-ying Chen, Jeffery Rest, Abdellatif M. Yacout, Marquis A. Kirk, and James F. Stubbins, Fission gas transport and its interactions with irradiationinduced defects in lanthanum doped ceria, presented at IBMM and accepted for publication in Nuclear Methods B.

Aaron Oaks, Benjamin Holtzman, Bei Ye, Di Yun, Wei-Ying Chen and James F. Stubbins, Kinetic Monte Carlo Model of Defect Transport and Irradiation Effects in U, UMo, and $\mathrm{UO}_{2}-$ type Fuels, presented at NUMAT October 3, 2010, accepted for publication in J. Nucl. Mater.

Bei Ye, Mark A. Kirk, Weiying Chen, Aaron Oaks, Jeffery Rest, Abdellatif Yacout, James F. Stubbins, TEM investigation of irradiation damage in single crystal $\mathrm{CeO}_{2}$, Journal of Nuclear Materials, In Press

A. Oaks and J. F. Stubbins, KMC Model of Vacancy Diffusion and Clustering in BCC Iron, Poster Presentation at the 15th International Conference on Fusion Reactor Materials, Charleston, SC, October 16 - October 22, 2011 Accepted for publication in Journal of Nuclear Materials.

A. Oaks, D. Yun, B. Ye, W. Chen and J. F. Stubbins, Kinetic Monte Carlo Model of Defect Transport and Irradiation Effects in La-doped $\mathrm{CeO}_{2}$, Journal of Nuclear Materials, Volume 414, Issue 2, 15 July 2011, Pages 145-149

Di Yun, Aaron J. Oaks, Wei-ying Chen, Marquis A. Kirk, Jeffrey Rest, Abdellatif M. Yacout, Zinetula Z. Insepov, James F. Stubbins, Kr and Xe irradiations in lanthanum (La) doped ceria: a study at the high dose regime, Journal of Nuclear Materials, Volume 418, Issues 13, November 2011, Pages 80-86

Wei-Ying Chen, Jianguo Wen, Mark A. Kirk, Yinbin Miao, Bei Ye, Brian Kleinfeldt, Aaron Oaks, James F. Stubbins, Characterization of Dislocation Loops in $\mathrm{CeO}_{2}$ Irradiated with High Energy Krypton and Xenon, submitted to Phil Mag 
Fundamental Studies of Irradiation-Induced Defect Formation and Fission Product Dynamics in Oxide Fuels

Yinbin Miao, Wei-Ying Chen, Aaron Oaks, Brian Kleinfeldt, James F. Stubbins The Nucleation and Growth Mechanism of the Dislocation Loop in Lanthanum Doped Cerium Oxide under Irradiation, in preparation

\section{Presentations}

B. Ye, M. Elbakhshwan, W-Y. Chen, Y. Miao, A. Oaks, B. Kleinfeldt, X. Han, B. Heuser, J. Stubbins, J. Rest, L. Yacout, M. Kirk, J. Terry, "Nuclear Fuels Irradiation Performance," presented at the IAEA Technical Meeting for the Enhancement of Accelerator Based Real-Time Methods in Development and Characterization of Materials for Energy Applications, Argonne National Laboratory, 24-28 January 2011, IAEA Technical Report F1-TM40585

Bei Ye, et al., "on The Influence of Temperature on the Evolution of Irradiation-Induced Defect Structure in Ce02," presented at TMS in Feb 2011 (won best student presentation award in the MPIM TMS Sessions).

Weiying Chen, Bei Ye, Mark Kirk, Aaron Oaks, Yinbin Miao, Brian Kleinfledt, Di Yun, James F. Stubbins, "The Stoichiometry Dependence of the Evolution of Irradiated-Induced Defect Clusters in Lanthanum Doped Ceria," presented at TMS in Feb 2011 (won runnerup best student presentation award in the MPIM TMS Sessions).

W. Chen, B. Ye, A. Oaks, Y. Miao, B. Kleinfeldt, M. Kirk and J.F. Stubbins, "TEM Observation of In-Situ Irradiations on $\mathrm{Ce}_{x} \mathrm{La}(1-\mathrm{x}) \mathrm{O}_{2}$ Single Crystal", Poster Presentation at APS/CNM/EMC Users Meeting, Argonne IL 2011

A. Oaks, D. Yun, B. Ye, W. Chen and J. F. Stubbins, Stoichiometric Dependence of Oxygen Diffusivity in $\mathrm{La}_{x} \mathrm{Ce}_{1-\mathrm{x}} \mathrm{O}_{2-\mathrm{x} / 2}$, Poster Presentation at the Minerals, Metal, \& Materials Society Conference, San Diego, CA, February 27 - March 3, 2011

Yinbin Miao, Aaron Oaks, Bei Ye, Wei-Ying Chen, Brian Kleinfeldt, and James F. Stubbins, Molecular Dynamics Simulation on Frenkel Pair Recombination Behaviors in Lanthanum Doped Cerium Oxide, Presentation at The ASME Applied Mechanics and Materials Conference, Chicago, IL, May 30 - June 1, 2011

Weiying Chen, Bei Ye, Brian Kleinfledt, Mark Kirk, Aaron Oaks, Yinbin Miao, James F. Stubbins, "Stoichiometry Dependence of the Evolution of Irradiated-Induced Defect Clusters in (Ce,La)O $\mathrm{O}_{2}$," presentation at TMS 2012 Conference, Orlando, FL, March 11 March 15, 2012

Yinbin Miao, Wei-Ying Chen, Aaron Oaks, Brian Kleinfeldt, and James F. Stubbins, The Simulation Approach of the Dislocation Nucleation and Growth Mechanism in Ceria under Irradiation, Poster Presentation at The ANS Annual Meeting, Chicago, IL, June 24 - June 28, 2012 
Yinbin Miao, Wei-Ying Chen, Aaron Oaks, Brian Kleinfeldt, and James F. Stubbins, Computer Simulation of the Dislocation Loop Evolution in Irradiated Ceria with and without Lanthanum Dopants, Poster Presentation at NuMat 2012, Osaka, Japan, October 22 - October 25, 2012

Wei-Ying Chen, Jianguo Wen, Mark Kirk, Yinbin Miao, Bei Ye, Aaron Oaks, Brian Kleinfeldt, James F. Stubbins Characterization of Dislocation Loops in CeO2 Irradiated with High Energy Ions, Poster Presentation at NuMat 2012, Osaka, Japan, October 22 October 25, 2012

Wei-Ying Chen, Yinbin Miao, Aaron Oaks, Brian Kleinfeldt, and James F. Stubbins "Defect Structure Evolution in Ion-Irradiated Ceria: Experiments and Modeling," NuMat , Osaka, Japan , October 22-25, 2012 [Oral, Keynote, Invited] 
Fundamental Studies of Irradiation-Induced Defect Formation and Fission Product Dynamics in Oxide Fuels

VI. THESES AND DISSERTATIONS

\section{MS}

Holtzman, Benjamin, MS, A new Age of Fuel Performance Code Criteria Studied Through Advanced Atomistic Simulation Techniques, advisor JF Stubbins. (2010)

Oaks, Aaron, MS, Development of Kinetic Monte Carlo Code to Study Oxygen Mobility in Lanthanum-Doped Ceria, advisor JF Stubbins. (2010)

Chen, Wei-Ying, MS, Stoichiometry Dependence of the Evolution of the Irradiated -Induced Defect Clusters in Lanthanum-Doped Ceria, advisor JF Stubbins. (2011)

Miao, Yinbin, MS, Computer Simulation of Defect Evolution in Lanthanum Doped Cerium Dioxide Under Irradiation, advisor JF Stubbins. (2011)

Kleinfeldt, Brian Robert, MS, Microstructural Investigations of Ion (Kr) Irradiated $\mathrm{UO}_{2}$, advisor JF Stubbins. (2012)

\section{PhD}

Yun, Di, PhD, Fission Gas Transport and Its Interaction with Irradiation Induced Defects in Lanthanum Doped Ceria, advisor JF Stubbins. (2010)

Ye, Bei, PhD, Formation and Growth of Irradiation-Induced Defect Structures in Ceria, advisor JF Stubbins. (2011) 
Fundamental Studies of Irradiation-Induced Defect Formation and Fission Product Dynamics in Oxide Fuels

VII. STUDENTS, DEGREES AND PLACEMENT

\begin{tabular}{|l|l|l|l|}
\hline Student & Degree & Date & Placement \\
\hline Benjamin Holtzman & MS & May 2010 & Westinghouse \\
\hline Aaron Oaks & MS & May 2012 & Continuing PhD Student \\
\hline Weiying Chen & MS & May 2012 & Continuing PhD Student \\
\hline Yinbin Miao & MS & May 2012 & Continuing PhD Student \\
\hline Brian Kleinfeldt & MS & May 2012 & Enercon \\
\hline Di Yun & PhD & May 2010 & ANL Scientific Staff \\
\hline Be Ye & PhD & May 2011 & ANL Postdoctoral \\
\hline
\end{tabular}

